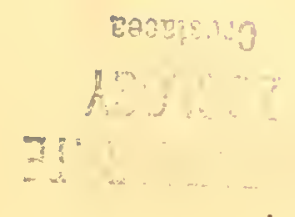

\title{
Crustace en.
}

Rearbeitet ron

\author{
Dr. A. Ortmann.
}

Abdruck

aus SEMON, Zonlngische Forschungsreisen in Australien und den malayischen Arehipel.

Verlag von Gustav Fischer in Jena.

1894.

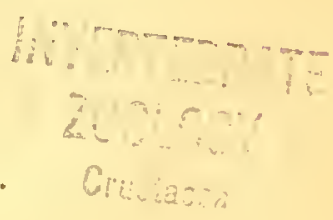








\section{Fundorte der von R. Semon in den Jahren $1891-1893$ gesammelten Thiere.}

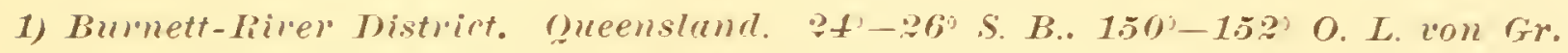

Es wurde am Mittellauf und Oberlauf des Burnett und an seinen Nebenflüssen Barambah Creek, Aranbanga Creek, Borne River, Auburn River. St. Johns Creek. Three Moon Creek gesammelt: rormiegend in der Gegend ron Coonambula. Cooranga und bei Gayndah.

August jogl bis Januar IS92. Juli bis October I892.

3) Hinterland ron Coolitom, Quemstamer. 15: S. B.. 145 O. L. ron Gr.

Bergland am Endeavour Rirer. Annan Rirer. Oaky Creek.

(Juni Is@2.)

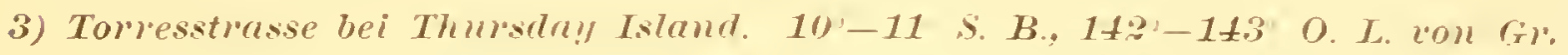

Seethiere wurden gesammelt in Aen Meerestheilen um Thursday Island und den Nachbarinseln (Prince of Wales Gruppe), ferner noch bei Double Island und Sirait Island und an der hüste des Fest. landes (Cap York, Jardine River). Die Landthiere stammen fast ausnahmslos ron Thursday Island. Die Süsswasserschneche Pseudopotamis semoni Martens wurde aut Hammond Island gefunden.

(Februar und März I 102.1

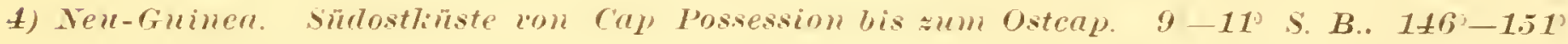
O. L. ron $\mathrm{rr}_{\mathrm{r}}$.

Der grösste Theil der Sammlungen stammt von Roro (Jule Island) und dem benachbarten Festland. ron Hula, Aroma. South Cape Suau und dem gegenüberliegenden Festland) und dem nordöst. lichen Teil der Milne Bay bis zum Ostcap. Eine Anzahl Paradiesrögel stammt rom Nordmesten ron Neu-Guinea, was an der betreffenden sielle besonders hervorgehoben werden wird.

(April und IIai IEg2.)

5) Insel Ambon. 3' +1 S. B.. 1:3 O. L. eon Gr.

Es wurde gesammelt in der Aussen- und Innenbai ron Amboina, in der Bai ron Baguala und der Bai ron Waai somie an den Küsten dieser drei Baien.

(Januar und Februar I\$93.)

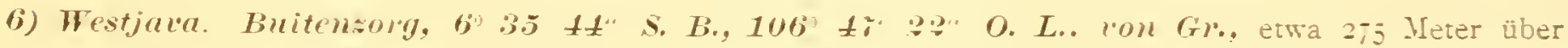
dem Meere. Tjibodas. I +23 Meter über dem Meere auf halber Höhe des Tulhans Gedeh.

INorember. December 1592. Mlärz IS03.1

Ausserdem wurden noch rereinzelte Exemplare auf Celebes. Menado, Gorontaio. Ternate und Batjan gesamael:: Planzion auch bei Banda, Makassar. Surabaya, Samarang, Penang und im Ganges bei Benares. 


\section{Crustaceen}

bearbeitet von

Dr. A. Ortmann.

Mit Tafel I-III. 

Die Hauptmasse der von Herrn Professor Dr. SEyox gesammeiten Crustaceen stamm von Amboina, eine reitere rosse Anzahl von der Thursday-Insel. Ton anderen Lobaliäien lJava. Celetes. leu.Guined. Queensland sind nur wenige Formen rorhanden. Die gesammien marinen Frmen gehören der lndo-Pacifischen Litoralfauna ${ }^{2}$ । an.

Im Winter I8go-gI hatte ich Gelegenheit. die Indo.Pacifische Crustacenfauna aus eigner Anschauung kennen zu lemen, und zwar an der ost-afrikanischen Küste auf der Strecke ron Zanzibar is Mikindani, besonders in der Lmgegend ron Dar-es-Salaam. Ich richtete damals meine besondert Aümerksamkeit auf das Vorkommen der einzelnen Krebsformen. aut die Facies ihrer Wohnpläize. auf die L'mgebung und die Gesellschaft, in der sie leben. Da einerseits auf diese lierhälnisse bisher wenig Rüchsicht genommen wurde, andererseits ron den ron mir dort beobachieten und gesammelien Formen marche identische und riele rerwandte sich auch unter dem ron Professor SEnor gesammelten Material finden. so ergaben sich eine Anzahl Beziehungen. auf die ich bei der Bearbeitung des letzteren Materials eingehen musste. Beziehungen, die in Zusammenhang stehen mit der Thatsache. dass eine in den wesentlichsten Zügen gemeinsame Litoralfauna sich ron der Ost-Küste Afrikas bis weit in den Pacinschen Ocean hinein erstreckt. Ich habe deshalb im Folgenden. mit Zustimmung ron Herrn Professor SEvor. bei Je Sammlungen gemeinsam bearbentet. Hierzu kommt noch eine kleine Anzahl Crusiaceen. die die zoologische Sammiung zu Strassburs ron Port Elisabeth /Capland erhielt, die an und für sich rech: interessant ist und besonders für die Begrenzung der Indo-Pacifischen Fauna nach Süden von Wichtigkeis wurde.

Die einzelnen Formen der Decapoden führe ich in derselben Reihentolge aus. die ich bei meiren Arbeiven über die Decapoden-Krebse des Strassburge: Huseums (Zoolog. Jahrt. Bd. V. Iago u. folg.l eingehalten habe. Ich habe es mir besonders angelegen sein lassen, bei neuea und weniger tehannten Formen die rerwandtschaftichen Bezichunger klar zu legen und meist dazu die Form ron Tatellen ischlüselas angewendet. Durch dieses Veriahren haffe ich mir den Dank der Fachgencssen zu rerdiener. da es jedenfalls unzweifelhaft ist. dass in dieser Treise allein die zahlreichen irüher teschriebenen Formen tritisirt werden können. Die Möglichkeit der Anfertigung ron Tabellen ist geradezu eire Píbe darauf. ob dic beschriebenen Formen in der Wreise charabterisirt Morden sind. dass man sie unter die ..tekannien" Arien einreihen kans.

Diese kritische Sichtung de: Crustaceen-S̈rstems ist eine unerlässliche Torbedingung darür. dass die geographische Verbreitung in richtiger Weise gew ürdig wird. Jedenialls ist es hlar. dass dadurch. dass verschiedene Arten unter einem Jamen zusammengeworfen werden. oder dadurch. dass eine Art unter rerschiedenen Jamen aufgerührt wird. falsche Bilder üter die geographische Verbreitung gewonnen werden. Aber nichi nur die Kritik des schon Bekannten isi iür den Ausbau der marinen Thiergeographie von Wichtigkeit. sondern auch die Erkenntniss der Vermandtschaitsbeziehunzen der einzelnen Formen. Es genügt durchaus nicht. zu konstatiren. dass hier diese, dort jene Form rorhommi. scadern es ist nothwendig. dass man ein Lrtheil darüber gewinnt. ob diese Formen in näherer Beziehung zu einander sichen oder nicht.

Schliesslich ist es für thiergeographische Siudien ein weiteres Eriorderniss. die Faunen besimmter Lokalitäten möglichst genau zu kennen. und diese Kenntniss wird nur durch fortgeseizies sammein erreich werden können. Insofern muss es mit Freuden begrüsst werden. dass die SEvcr"schen sammiungen aus im Grossen und Ganzen zut bekannien Gebieten herrühren. da man aus ihaen die Leberzeugung sewirni. dass es selbst in diesen qut behannten Gezenden noch manche stsiematische und thiergecgraphishe Jeu. heiten giebt. welche bisher den Sammlern entqangen waren. Téder einzęhe derartige Funf bunn unier

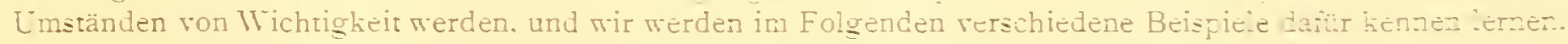

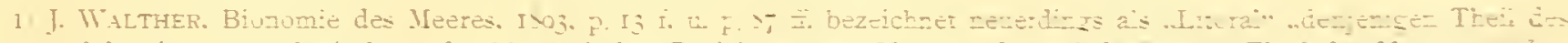

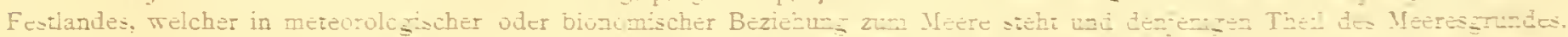

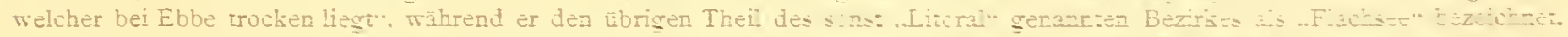

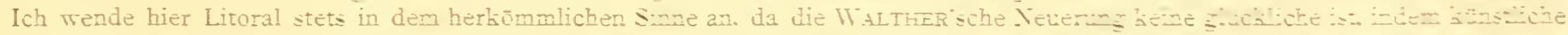

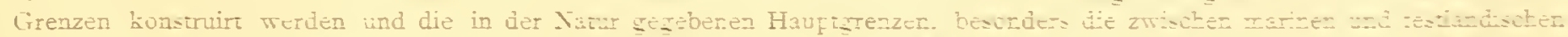

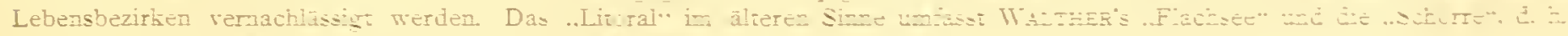
den Theil des Meerestundes, der bei Ebot trocien İ̈i. 


\section{Vel'zeichniss der an den einzelnen Fundorten gesammelten Arten.}

\section{Coll. Semon.}

Penaeus cannliculatus OLIV. Caridina typus I.-E.

C. brevicarpalis D. II. Atya moluccensis D. $\mathrm{H}$.

A. brexirostris D. II.

Alpheus eduardsi (AtD.)

A. lobidens D. H.

A. laevis RAxid.

A. frontalis $\mathrm{S} \mathrm{I}$.

Hymenocera elegans HELL.

Palaemon lar FABR.

$P$. dispar r. MLART.

Stenopus hispidus (OLIv.)

St. tenuirostris D. $\mathrm{II}$.

Panulirus polyphagus (HBst.)

Puer spiniger ORTM.

Scyllarus haani D. H.

Enoplometopus pictus A. M.-E.

Thalassina anomala (HВST.)

Gebia barbata STRAHL

G. intermedia D. M.

Callianassa mucronata STRAHL

Galathea elegans AD. WH.

Penaeus monodon FABR.

Alpheus edwardsi (ALD.)

A. strenuus Dax.

$A$ villosus (OLIv.)

A. comatularum HASw.

A. biunguiculatus STIMPS.

Panulirus polyphagus (Hвsт.)

Gebia hexaceras ORTM.

Pachycheles putchellus (HAsw.)

Polyonyx biunguiculatus (DAx.)

Pagurus imbricatus .I.-E.

Diogenes avarus HELL.

Cryptudromia lamellata ORTM.

Oreophorus rugosus STIMPS.

lxa cylindrus (FABR).

Myra affinis BELL.

\section{Amboina.}

Mnnida semoni ORTM.

Pagurus munctulatus OLI.

$P$. enopsis DAx.

P. deformis M.-E.

P. asper D. H.

Eupagurus hirtimamus Mrs.

Coenobita clypeatus (HBST.)

C. rugosus M.-E.

C. compressus M.-E.

Birgrs latro (L.)

Remipes testudinarius LATR.

Dynomene hispida DEsM.

Calappa hepatica (L.)

Iatuta victrix FABR.

Myra fugax (FABR.)

Lencosia perlata D. H.

Camposcia retusa LATR.

Schizophrys aspera (M.-E.)

Tiarinia gracilis DAx.

Tylocarcinus styx (HBst.)

Carupa laeriuscula HELL.

Teptumus pelagious (L.)

I. sanguinolentus (Hвst.)

\section{Thurstay Island.}

Tursia ypsiton ORTM.

Lencosin perlata D. H.

Achrews affinis Mrs.

Camposcra retusa Latr.

Anacinetops stimpsoni Mrs.

Huenia proteus D. II.

Pseudomicippe varians MRs.

Egeria arachnoides (RPH.)

Hyastenus diacanthus (D. H.

H. sebae $\mathrm{ITH}_{\mathrm{H}}$

Naxia serpulifora (ivér.)

N. cerastes ORTM.

Micimpe thatia (HBST.)

Neptunus pclagicus (L.)

Goniosoma sexdentatum (HBsт.)

Thalamita sima M.-E.
Thalamita admete (HRST.)

Th. pryma (HEST.)

Lambres hoplonotus AD. WH.

Atergatis floridus (I.)

Phymodius ungulatus (.I.-E.)

Carpilodes raillantianus A. II.-E.

Carpitius comerers (Foksk.)

Chlorodopsis melanochirus A. M.-E.

Dairn perlati (HRst.)

Cycloblemas semoni ORTM.

Eriphia laevimana IstTR.

Trapezir cymodoce (Hвsт.)

Tr. "ufomurtata (H Bst.)

Leiolophus abbreriatus (Dsx.)

Myctiris lomgicarmus LATR.

Lysiosquilla maculata (FABR.)

Psendosquilla ornata Mrs.

Gonodactylus scyllurus (I.)

G. chiragra (FABR.)

G. stoliurus (MtELL.)

Lepras amatifera I.

$L$ anserifera 1 .

Tetraclita porosa (L.)

Thalamita intermedia MRs.

Th. stimpsoni A. M.-F.

Th. crenata (1.atTh.)

Lambras lecanora URTM.

Cryptopodia spatulifions Mrs.

Gonatonotus pentugoms $\mathrm{WH}_{\mathrm{H}}$

Atergatis floridus L.)

Actumnus sctifer (D. II.)

A. pulcher (.Mrs.)

Metopograpsas messor (Fonsk.)

Macrophthalmus telescopreus (Ow.)

Ocypode cerutophthalmin (PALL.)

Gonodactylus uraphurus Win.

Tetraclita cocrulescens (SPGL.)

Pyrgoma cancellatum LEACH. 
Milimalrmi.

Sesarma erythrodactyla IIEss.

Plugusia immaculato LAM.

Leiolophus abbrevintus DAX.)

Lepres anatifern I.

Balanus tintinnabulum I.

Tetraclita porosa (I.

I'hurichilus moridionalis börm.
Limrli.

Coenobitu compressus M.-E. Cardisoma carmifex II Bst.) Gelasimus cultrimanus Wи.

G. urvillei M.-J:

$G$. inversus HoFry.

G. annulipes MI.-E.
Somgre-Songr-Iusel.

Deypode bulli D. H.

O. cerratophthalma (I'ALL.)

Zrrnzibrer.

Gelasimus amulipes M.-E.

\section{Port Elisabeth.}

Scyllarus elisabethe ORTM.

Gebia africana ORTM.

Pseudorlromia latens STImp'

Mursin cristata LatR.

Hymenosoma orbieulare DEs.
Acanthonyx dentatus M.-E.

Maja squinado (IROND.)

Portummus pulchellus (MACL.)

Platyonychus bipustulatus M.-E.

Pilumnes infraciliaris ORTM.
Lophozozymus dodone (II BST. Eriphia smithi MACL. Cyclograpsus punctretus II.-E. Ocypode kuhli D. H.

o. ceratophthalma (PALL. 
Teu Guimer.

Palneman lar FABR.

Ponulirus poilyg-hagus । HBsT.।

Coenctita rugists .I.-E.

C. compressus M.-E.

Sesarma oracilige: II.-E

Penaeus conaliculatus ULIr.

F. monoceros FABP.

P. monsdor FABR.

P. inäieus II.E.

Caridina vecti HICES.l

Athanas dimorphus OPTY.

Atpheus educardsi A[D.l

A. maerochirus RICHT.

A loeris Pusp.

$\therefore$ graciliqes STIMF:

Saron marmoratus (OLIT.)

Pintonia jinnae ORTM.

Coralliocaris graminea I DAs.

Anchistia ensifrone D.x.

Learider lengicaryus STIUPE.

Falaemon idae HELL.

Petraisthes laniarchi (LEACH.)

F. tricirgatus Dr.tM.

Pisisoma granulatum ORIM.

Pachycheles sculptus II.-E.)

Pagurus punctulatus OLIt.

P. setifer M.-E.

P. dejormis Y.-E.

Cibanarius langitarsis (D. H.)

C7. rirescens / hiptes.

Calcinus $\}$ erlesti D. II.

C. gaimardi H.-E.

Tiggenes ararus HELL.

Coenotita clypentus 'HBsT.)

C. ruggsus II.E.

C. compressus II.-E.

C. perlatus M.-E.

Iromidia unidentata ( $\mathrm{PL} \bar{L}^{\mathrm{PP}}$.)

Cryptodromia pentrgomalis HILG.

Calapya hepatica I.

Matuia vicirix FABP.

Alyheus educardsi . A LัD.

A lobiders D. H.

C'ibanarius tireecens ( hipates)

Calcirus herbsti D. Y.

Coenotita comyressus .I.-E.

Calapra hepaica I L.

Ixa cylindrus FABR.।

Iyra fugar FAER.

\section{Burnett.}

Miersin compress D. H.

Caridina erecki HICES.

Falaemor ausiralis UPTY.

Cherate Gicarinatus lipaI.

Celebes.

Lepre anserijera $L$.

\section{Coll. Ortmann.}

\section{Dar-er-salaam.}

Phitura ylatycheira D. H. Huevia arandidier A. M.-E.

Uerathius monoceros (LATR.)

Acanitionyx quadridentatuz hracss.

Pseudomicipye modasa HELL.

Ticipge philyra IHE:T.

Tylgcarcinus siyx HEミT.

Teptune aranulatue (II.-E.।

1. sanouinolentus (HEミT.)

Scylla serrata (Fors $\mathrm{E}$.

Gonigsoma aifine IDAs.

G. darae A. I.-E.

Thalamita sarignyi A. X.-E.

Th. crenata I LATR.।

Lambrus yisoides AD. WH.

Yyomenippe panape HBミT.I

Fseudozius cuystrus (AD. $\mathbb{H}_{\mathrm{H}}$.

Eurycarcinus natalenses (hipats.)

Pilumius respertilio (FABR.)

Cuma aridreossyi is A T.

Iantho esaratus (M.-E.।

Actrea tomentosn I.M.-E.।

1. rufopunctata (M.-E.)

1. caripes (I) 1 .

Zozymus aeneus IL.I

Lophactrea cristata A. M.-E.

Phymodius ungulatus 1.M.-E.

Chilorodius niger (Fors.s.)

Hypocoelus sculptus (M.-E.)

Carqiludes tristis DAN.

C. raillantianus A. M.-E.

C. moniticulozus A. M.-E.

Carpilius contexus (Forsk.)

Ozius rugulasus STIMPs.

Episanthus corrosus A. V.-E.

\section{Filna Fininrlje.}

Leucusin margaritacea HELL.

Stenorhynchus uretis roru.

Paratymolus pubtescens MRs.

Huenia grandidieri A. M.-E.

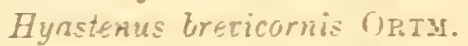

Gonicasuna danae A. W.-E.

Thalamita crenata (LATR.)

Lambrus pelagicus HËPP.
Jara (Buitenzorg u. Thibodas).

Palaemon eleoans D. M.

Paratelyhusa tridentata M.-E.

Telphusa kuhli D. II.

Sesama nodulifera D. II.
Epicantius dentatus (WH.)

Eurīppellia amulipes (M.-E.)

Eriphia laecimama LaTR.

E. smithi MaCL.

Traperin cymudice (HBsT.)

T. rwopunctata (HBEI.)

Tetralia gleterrima (HBST.1

Metopgrapsus nessor Forsz.।

3. acenricus (JACQ. LCC.)

Grapsus grapins I.

Gr. strigusus (HBミT.)

Taruna Titterato (FABR.)

Heteragraysus erythraeus (Kossu.)

Sesarma elongata A. M.-E.

S. meinerti D. YI.

S. erythrodactyla HESS.

Helice leachi Hess.

Leioloyius abbretiatus (D.s.y.)

Cardisumn carnifex (HBsT.)

Euplax boscii (AtD.)

Macrophthalmus grandidieri A. M.-E.

Dotilla fenestrata HiLG.

Felasimus cultrimanus $\Pi_{\mathrm{H}}$.

G. urcullei I.-E.

$G$. intersus HOFFM.

$G$ annulipes II.E.

Ocyugde liutili D. H.

O. ceratophthialma (PALL.)

Gurodactylus chiragra (FABR.)

G. glaber BP.

G. nov. spec.

G. trispinosus $\mathrm{WH}_{\mathrm{H}}$

Lepas anatifera L.

Tetraclita porasa (L.)

Figrgoma milleporat DAF.T.

Tantho exnratus (U.-E.)

Eriphia smithi IIACL.

Incruyhthalmus grandidieri.A. M.-E.

IJotilla feriestrata HILG.

Gelasimus cultrimanus $\mathrm{WH}_{\mathrm{H}}$

G. anmulipes V.-E.

roypode Tiuhli D. H.

o. ceratophthalma PALL.) 


\title{
Decapoda.
}

\author{
Familie: Pellatidae.
}

\section{Penaeus camaliculatus Otivier.}

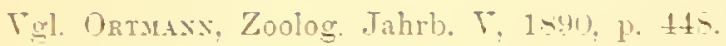

Amboina, I $\delta$ ad. (SEMON coll.).

Dar-es-Sal a a m. Ras Rongoni. I f jur. (ORTMAxx coll.), bei Ebbe in Wasserlöchern auf sandigem Grunde.

Verbreitung: Indo-Pacifisches Gebiet ron Ost-Afrika und Mauritius bis Japan, Sydney und Tahiti. - Ton Ost-Afrika ron Zanzibar und Mozambique angegeben (HrLgexdorf): ron Amboina durch DE MAX bekannt.

\section{Penneus monoceros FABRICILs.}

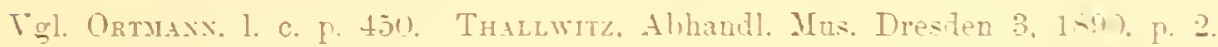

Dar-es-Salaam. $3 \delta$ t alle jung, wurden mir ron Negern gebracht und stammen wohl ron der Mündung des Lpanga-Flusses her.

Verbreitung: Indo-Pacifisches Gebiet, von Ost-Afrika bis Japan und Australien. - Ost-Atrikit: Quilimane (Hilgexdorf).

\section{Penacus momodon Fabricits. Taf. II, Fig. I.}

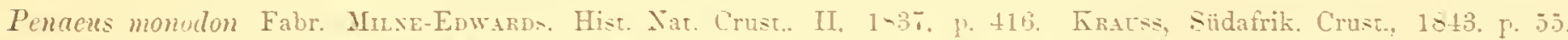

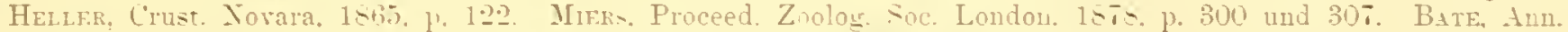

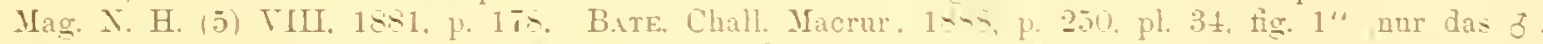

P. semisulcatus exsulcatus Hingrinur, Mon. Ber. Akad Tiss. Berlin. 1ET- H. 843.

Mir liegen zwei Exemplare aus Ost-Afrika und eines ron der Thursdar-lnsel ror, die dem P. semisulcatus sehr nahe stehen, aber rom typischen semisulcatus gewisse Abweichurgen zeigen, und für die ich den Namen P. monodon FABR. angewendet wissen möchte. Dieselben stimmen röllig mit HilgExDorf's Beschreibung des semisulcatus rar. exsulcatus überein, d. h. die Furche des Rostralkieles ist kaum bemerhbar. Die Bezahnung des Rostrums ist bei den afrikanischen Exemplaren: $\frac{7}{3}$, bei dem von der Thursdar-Insel: $\frac{5}{3}$, während Exemplare des semisulcatus von Japan meist z zeigen. was für die Zugehörigkeit meiner Exemplare zu P. monodon nach Miers" Fassung sprechen würde.

Nun finde ich aber ferner eine wesentliche Abweichung in der Bildung des Thelycum bein $\ldots$. Nach dieser würden die vorliegenden $?$ in meine Gruppe A. B. B. B. (Zool. Jahrb., V, I8go, p. fłi) gehören. d. h. die letzte harte Spange des Sternums ragt zwischen die 5. Pereiopoden in zwei Lappen nach vorn. Ob das Petasma des ¿ T-förmig gebildet ist, kann ich nicht angeben, da mein $\delta$ zu jung ist. Jedenfalls ist es srmmetrisch gebildet.

Diese Art würde sich von den beiden anderen in die genannte Gruppe gehörigen courirostris und crucifer) unterscheiden: I. Auch das Ischium des 1. Pereiopoden besitzt einen Dorn. 2. Rostrum nach hinten in einen Kiel fast bis zum Hinterrand des Cephalothorax fortgesetzt. Dieser Kiel ist schwach oder nicht gefurcht. 3. Rostrum am Unterrand gezähnt. +. Die beiden Lappen der letzten Spange des Sternums beim $q$ bis zur Basis der 4 . Pereiopoden reichend.

Ton den Abdomensegmenten sind die drei letzten gehielt.

Thursday Island. I $\rightleftharpoons$ (SEnor coll.).

Dar-es-Salaam, Lpanga-Riff, I : im Hafen bei Itoni, I 3 jur. (OrTmaxx coll.).

Verbreitung: Miers giebt für seinen monodm Indian and Australian seass an. genauer (p. 200): Australien, Shark-Bay und Ceỹlon. - Ferner: Zanzibar (Hilgexdorf); Quilimane (Hilgexdorf); Natalküste (Kralss); Cerlon (Helder); Neu-Gninea (Bate). 
Penaeus indicus MLNE-F.DWARDS.

Mitue-Edwards, Hist. Nat. Crust., II, 1837, p. 415. Miers, Proceed. Zool. Soc., London 1878. p. 301 und p. 307. Hilgexdonf, Ion. Ber. Ak. Wiss. Berlin, 1878. p. 844. Bate, Ann. Mał. N. H. (5), TIII, 1881, p. 177, pl. 12, fig. 5. Bate, Chall. Macrur., 1888, p. 248, pl. 33, fig. 2

Steht dem $P$. monorlon sehr nahe, unterscheidet sich aber: I. Rostrum schlanker und länger, doch scheint Länge und Bezahnung etwas zu variiren. Bei meinen Exemplaren überragt es eben die Antennenschuppe, die Bezahnung ist: $\frac{T}{3}$ und $\frac{7}{4}$. 2. Furchen zur Seite des Rostralkieles kaum angedeutet, oder völlig fehlend. 3. Die gegen die Fühlerschuppe gerichtete horizontale Furche auf der Seite des Cephalothorax ist bei monodon sehr scharf, bei indicus fehlt sie: sie wird nur durch einen undeutlichen Kiel angedeutet. 4. Geisseln der inneren Antennen, bei indicus etwas länger. 5. Thelycum ähnlich wie bei monodon, jedoch ragen die beiden Lappen der letzten Spange des Sternums weniger weit vor, nicht über die Basis der 5. Pereiopoden hinaus ${ }^{1}$ ).

Ueber das Petasma vgl. BAte, 1888, pl. 33, fig. 2 p.p.

Dar-es-Salaam, 2 ; mit $P$. monoceros erlalten.

Verbreitung: Quilimane (Hilgendorf); Coromandel (Mrlne-Enwards); Ceylon (Miers); Singapur (Dana); Java (Heller): Batavia (Hhlgendorf); Chefoo (Miers); Amoy (Miers); Philippinen (BAte).

Familie: Atyidae.

Die Gattungen der Atyidae lassen sich nach folgender Tabelle unterscheiden:

a. Sämmtliche Pereiopoden mit Exopoditen. Carpus des I. Pereiopoden nicht oder nur undeutlich ausgehöhlt.

Miersia ${ }^{2}$ ).

aa. Nur die beiden ersten Pereiopodenpaare mit Exopoditen. Carpen der 1. und 2. Pereiopoden am distalen Ende ausgehöhlt.

Hemicaridina.

aaa. Pereiopoden ohne Exopoditen.

b. Carpus des 2. Pereiopoden von normaler Gestalt. Rostrum (meist) comprimirt und gesägt.

bb. Carpus des 2. Pereiopoden ebenso wie der des ersten am distalen Ende ausgehöhlt. Rostrum (meist) nicht comprimirt, nicht gesägt. Atya.

\section{Miersia commressa (DE HAAN).}

Vgl. Ortuaxi. Zool. Jahrb. T, 1890, p. 463.

Die Scheeren der I. Pereiopoden sind insofern von normaler Gestalt, als der Carpus an distalen Ende nicht oder nur ganz undeutlich jene eigenthümliche Aushölung zeigt, welche die drei anderen Gattungen dieser Familie charaliterisirt. Jedoch zeigen die Finger der Scheeren die belianten, den Atyidae zukommenden Haarbüschel.

Nach dem Bau der I. Pereiopoden und dem langen Basalstachel der inneren Antennen möchte ich vermuthen, dass Caridina serratirostris DE MAN (in: WEBER's Reise, II, I892, p. 382) auch in diese Gattung gehört.

Queensland, Burnett, I Ex. (SEMon coll.).

(v. MARTENS).

Verbrejtung: Japan (de Haan, v. Martens, Miers, Ortuann); Insel Adenare bei Flores

\section{Caridiul typus MiLnE-EDwARDS.}

Caridina typus Milxe-Edwards, Hist. Nat. Crust., II, 1837, p. 313, pl. 25 bis. tị. 4. 5 ; Richters, Beitr. Meeresfaun. Maur. Seychell. Decap., 1880, p. 162, pl. 17, fig. 23; nE MAx, in: Weber's Zuol. Ergebn. Reis. Niederl. Ost-Ind., II, I892, p. 367, pl. 21, fig. 22; DE MAx, in: Tot. Leyd. Mus., 15, 1893, p. 300.

C. siamensis Greber, Zeitschr. f. d. ges. Naturw., 21, 1863, p. 329.

1) Dieser Befund stimmt nicht mit BATE (1. c. 188 r, pl. 12, fig. 5 und p.) überein.

2) Die Merkmale der Gattuug Kiphocaris v. Mantens (Arch. f. Naturg., 3S, 1, 1S72, p. 139) stimmen vollkommen mit Miersia Kingsley (Proceed. Acad. Nat. Sc. Philadelphia, I879, p. ji6) überein. (Vgl. auch: Kingsley, ibid., p. 426, u. Pocock, Annal. Mag. Nat. Hist. $(6$, III, 1889, p. 17.) Sollten beide wirklich, wie ich vermuthe, identisch sein, so würde Xiphocaris die Priorität haben. Die Arten von Tiphocaris sind west-indisch und kommen auch dort, wie aus den Bemerkungen bei Pocock (l. c. p. 6 u. 20 ) hervorgeht, in Süsswasser vor. - Von den von KixGsLEx für Miersiu angegebenen Typen gehören die beiden ersten (pelagica u. punctulata) wahrscheinlich nicht hierher. Die Gattung kommt auch in Neu-Seeland vor; im Strassburger Museum befinden sich 3 Exemplare einer noch unbeschriebenen Art aus dem River Avon bei Christchurcl. 
DE Max, l. c. p. 369.

"Rr. longrirostios DE MAN.

Der Name der Varietät ist schlecht gewählt, da schon eine Caridina longirostris MILNE-EDwARDS existirt.

Amboina, I Ex., Süsswasser (SEmon coll.).

Verbreitung der C.typus: Seychellen und Mauritius (Richters); Siam (GIebeL); Flores, Saleyer, Celebes, Timor, in Flüssen (DE MAN).

Carillua brevicurvalis DE MAN.

de Max, in: Weber, Zoolog. Ergebu. etc., II, 1892, p. 397, pl. 24, fig. 30.

Amboina, I Ex., Süsswasser (SEmon coll.).

$V$ erbreitung: Bisher nur von Celebes, aus Süsswasser bekannt (DE MAN).

\section{Caridina "ryel:i (Huckson).}

Atya wyckit Hickos. Annal. Mag. Nat. Hist. (i., II, 1888, p. 357, pI. 13, 14.

Carilina wyckii Thaldwtz, Abhandl. Mus. Dresten, 3, 1891, p. 27. de Max, in: Werer. Zool. Erg. ete., II, 1892, p. 386 , pl. 24, fig. 29 -29 k. de Max, Not. Leyd. Mus, 15, 1896 , p. 302, pl. 8, fig. 7 (var.).

Die vorliegenden Exemplare stimmen alle mit den unter $\mathrm{A}$. beschriebenen typischen Exemplaren DE MAN's überein.

Die von mir aus Ost-Afriha mitgebrachten Exemplare gehören hierher, und nicht zu longirostris Mulne-Edwards (Hist. Nat. Cr., II, i837, p. 363), die mit nilotica (Rotx) (Annal. Sc. Natur., 28, i833, p. 73, pl. 7, fig. I) identisch sein soll. Die Originale der longirostris (von Oran) unterscheiden sich nach DE MAN (1. c. p. 396) durch gedrungenere und kürzere Carpen der Scheerenfüsse. Es wäre möglich, dass die von Hilgendorf (Mon. Ber. Akad. Berlin is, 8, p. \$28) von Mozambique und Tette, von Pfeffer (Jahrb. Hamburg. Wiss. Anstalt, VJ, IS89, p. 35) von Zanziluar angeführte C. nilotica, sowie die von RICHTERs (Meeresf. Maur. Seych., I8S0, p. I62) von den Seychellen (n i cht von Mauritius, wie DE MaN 1. c. p. 397 irrthümlich citirt) angeführte C. longirostris ebenfalls zu C. wycki zu ziehen sind.

Queensland, Burnett, viele Ex. (SEmon coll.).

Dar-es-Sala am, 3 Ex., mit Penaeus monoceros und indicus erhalten (OrTMAxn coll.)

Verbreitung: Celebes (Hickson, Thallwitz, DE MAN), in Bächen und Flüssen; Ins. Saleyer, Fluss Banckalan (DE MAN); Flores, in Flüssen (DE MAN); Timor (DE MAN).

Die Grenzen des Verbreitungsgebietes dieser Art werden durch die obigen Funde ganz bedeutend erweitert: der Nachweis ihres Vorkommens in Ost-Afrika macht es höchst wahrscheinlich, dass die Angaben von Hilgendorf, Richters und PFefFer ebenfalls auf diese Art zu beziehen sind ${ }^{1}$ ). Die echte C. longirostris wird von Oran (MLLNE-EDwARDs) und die nilotica aus dem Nil (Roux) und von N.-O.-Afrika (HulegeDORF) angegeben.

Es sei hier gestattet, eine weitere neue Art der Gattung Caridina zu beschreiben, die durch die Bildung des Rostrums wesentlich von den vorangehenden Arten sich unterscheidet.

\section{Coridima simghatensis nor. spec. Tafel I, Fig. 2.}

Körper gedrungen. Rostrum sehr kurz, kïrzer als die Augen, stumpf dreieckig, flach, nicht comprimirt, oben mit einem kurzen, undeutlichen Kiel, ohne jede Zähne. Vorderseitenrand des Cephalothorax ohne Antennalstachel.

Carpus der I. Pereiopoden distal verbreitert und ausgehöhlt, kaun so lang wie die Scheere, sein oberer Rand etwas länger als die Breite am distalen Ende. Scheerenfinger etwa so lang wie die Palma.

Carpus der 2. Pereiopoden langgestreckt, länger als die Scheere, gegen das distale Ende nur wenig verdickt. Scheeren schlanker, Finger länger als die Palma.

Nerus der 3. und 4. Pereiopoden beim $\delta$ am Unterrande, etwa $\frac{1}{3}$ seiner Länge vom distalen Ende entfernt, verbreitert und eckig vorspringend, daselbst mit einem Stachelchen. Beim q fehlt diese Verbreiterung. Krallen am Unterrand mit 5-6 Dörnchen, nicht ganz ein Viertel so lang als der Propodus. Krallen der 5 Pereiopoden am Unterrand mit zahlreichen, feinen, kammförmigen Dörnchen, fast ein Drittel so lang als der Propodus.

1) Im Mus. Strassburg finden sich 6 Exemplare von Ceylon, Trincomali, die auch zu C. mycki zu rechnen sind: bei ihnen zeigt der Unterrand des Rostrums 6-9 Zähne, also weniger als bei typischen Exemplaren, und es stehen 3-4 Zähnchen des Oberrandes noch auf dem Cephalothorax. In allen übrigen Merkmalen stimmen sie aber mit typischen Exemplaren von wycki überein. 
Telson oben mit $6-8$ Dörnchenpaaren.

Die Eier der 9 sind auffallend gross und gering an Zah!.

Steht der C. brevirostris Stunpson (Proc. Acad. Nat. Sc. Philadelphia 1860, p. 9\$) von den BoninInseln sehr nahe, unterscheidet sich aber durch die Scheerenfinger der I. Pereiopoden, die so lang wie die Palma sind. Bei brevirostris sollen die Finger viel kürzer als die Palma sein.

Diese beiden Arten, brevirostris und singhalensis, bilden eine besondere Gruppe der Gattung, ausgezeichnet durch völlig ungezähntes, kurzes Rostrum und den Mangel des Antennalstachels.

Ceylon, Newera Elya, in Süsswasser, viele Exemplare, darunter aber nur I d. Die Originale befinden sich im Strassburger Museum.

Atya molnecensis DE HAAN.

Tgl. de Max, in T'Eber: Ergein. Reis. Niederl. Ost-Ind., II, 1892. p. 357, 11. 21, fig. 20.

Amboina, I q, Süsswasser (SEmor coll.).

Im Indischen Archipel fast überall: Sumatra, Jara, Celebes, Saleyer, Timor, Flores, Bali, Ceram, Batjan, Philippinen.

Atya brevirostris DE MAN.

IE MAx, 1. c. 1892 , p. 360 . pl. 21, fig. 21

Amboina, 3 , Süsswasser (SEMox coll.).

Verbreitung: Flores und Timor (DE MAx).

Familie: Alpheidae.

Gattung: Athamas LEACH.

Die Gattung Athanas gehört in die Familie der Alpheidae aus folgenden Gründen: I. Mandibel zweiteilig, mit Synaphipod. 2. Rostrum schwach entwickelt. 3. Erstes Pereiopodenpaar kräftiger als das zweite. Letzteres mit gegliedertem Carpus. 4. Mastigobranchien (Epipoditen) auf $k$ bis $n$ vorhanden. Exopoditen auf den Pereiopoden fehlend. 5 . Telson breit am Ende.

Aus der Diagnose der Alpheidne ist zu streichen, dass die Augen vom Cephalothorax tiberwölbt sind.

Von Alpheus unterscheidet sich Athunas: I. Augen mit der Cornea unter dem Cephalothorax vorragend. 2. Aeusserer Faden der inneren Antennen zweitheilig. 3. Erste Pereiopoden nicht sehr ungleich und nicht so auffallend gestaltet wie bei Alpheus.

Bisher waren nur drei Arten der Gattung Athanas bekannt: A. nitescens (LEACH) von den Küsten Europas und den Cap Verden, A. veloculus Bate (Chall. Macrur., p. 529) von den Cap Verden, und A. mascarenicus Richters (Beitr. Meeresf. Maur. Seych. I880, p. I64) von Mauritius. Die vorliegende Art ist die zweite, die von der Gattung aus den Indo-Pacifischen Gebiet bekannt wird und unterscheidet sich von mascarenicus durch den Bau der Scheerenfüsse, durch 5-gliedrigen Carpus der 2. Pereiopoden und durch das auf der Oberfläche bedornte Telson.

\section{Athamas dimorphas nov. spee. Tafel I, Fig. I.}

Cephalothorax gleichmässig gewölbt, Rostrum einfach, spitz, gerade vorgestreckt, bis zum 2. Stielgliede der inneren Antennen reichend. Supraorbitaldornen fehlend. Dorn an der äusseren Ecke der Augenhöhlen stark entwickelt. Augen nur mit der Cornea vorragend. Innere Antennen mit langem Styloceriten; ihre äussere Geissel an der Spitze zweitheilig, der dickere Theil kurz, mit Sinneshaaren, der dünnere länger. Aeussere Antennen mit ovaler Schuppe, letztere etwa so lang wie der Stiel der inneren Antennen.

Dritter Maxillarfuss mit Exopodit, dieser letztere auf allen Pereiopoden fehlend. Dagegen sind auf dem 3. Maxillarfuss und auf den vier ersten Pereiopoden ( $k$ bis $n$ ) Mastigobranchien vorhanden.

Erste Pereiopoden beiderseits gleich, beim of und $q$ auffallend verschieden gestaltet. Beim $\delta$ ist der Merus kräftig, lang, so weit wie der Stiel der äusseren Antennen reichend. Carpus kurz. Palma lang, etwa cylindrisch, viel länger als der Carpus. Finger liurz, der bewegliche gekrümmt, mit einem Zahn an der Basis der Schneide. Beim $q$ ist der Merus schlanker, der Carpus ebenfalls schlank, mindestens ebenso lang als der Merus. Die Scheere ist kurz, kürzer als der Carpus.

Zweite Pereiopoden schwach; Carpus 5-gliedrig, das erste Glied fast so lang wie die übrigen zusammen, das 2., 3. und 4. sind liurz, das 5. etwas länger. Die übrigen Pereiopoden sind gleichmässig.

Telson breit abgestutzt, oben mit zwei Dömchenpaaren. Aeusserer Ast der Schwanzflosse mit einer queren Naht vor der Spitze. 
Körpergrösse etwa $\mathrm{I} \frac{1}{2} \mathrm{~cm}$.

Dar-es-Sala am, Upanga-Riff, I $3+$, in Löchern und Höhlungen des Korallkalkes.

\section{Alphens edmermisi (Aunours).}

Sinonyme siehe bei: A. eduardsi (Alm., Mieks, Rep. Zool. Coll. Alert 1884, p. 284, ve MAx, Areh. f. Naturg., 53, 1 , 1857, p. 5lti. ne Hax, Journ. Linn. Soc. London. Zool. XXII, 1888, p. 26b. Ortmax, Zool. Jahrb.. V, 1840, p. tio. Ortmaxx, Ergebn. Plankton-Exp. Dec. u. Schiz, 1893. p. 44.

A. hani, Ortmaxx, Zool, Jahrb. T, 1890 , p. 4 i2.

A. macrolactylus, ORTMasx, ibid. 11. 473, 11. 36, tig 10

Die mir von Ost-Afrika, Amboina und der Thursday-Insel vorliegenden Exemplare entsprechen der von mir (ISgo 1. c.) als A. hami von Japan, sowie der (I893 l. c.) als edwarlsi var. von Bermuda angeführten Form. Ausserdem halte ich den A. macrodartylus ebenfalls für eine in den Lreis des A. euluartsi gehörige Form, während A. dolichodactylus ORTM. und acanthomerus ORTM. (identisch mit edamensis DE MAN) als besondere Arten anzusehen sind. Die Arten der Gruppe des A. edwardsi, in meiner Tabelle (1. c. I890, p. 468) die Abtheilung: A. B. C. D. E., also die Formen mit kurzem, schmalem Rostrum, mit rudimentärem Dorn am ersten Stielglied der äusseren Artennen, mit eingekerbtem Ober- und Unterrand der grossen Scheere und mit unbewehrter Augendecken, bei denen der bewegliche Finger der kleinen Scheere keine besonderen Haarleisten besitzt, würden sich nummehr in folgender Weise zusammenstellen lassen:

A. B. C. D. E.

F. Merus des 3. und 4. Beinpaares ohne Dorn. Erstes Carpalglied des 2. Beinpaares das längste.

G. Finger der kleinen Scheere mittelmässig oder kurz, zusammenschliessend.

A. edwardsi.

GG. Finger der kleinen Scheere sehr lang, fast so lang wie die ganze grosse Scheere, klaffend.

FF. Merus des 3. und †. Beinpaares mit einem Dorn.

A. dolichodactylus OrTm. Japan.

G. Erstes Carpalglied des 2. Beinpaares das längste.

A. hippothö̈ DE $\operatorname{MAN}^{1}$ ).

GG. Zweites Carpalglied des 2. Beinpaares das längste.

A. ellamensis DE $\mathrm{MAN}^{2}$ ).

Die letztere, von Java und Amboina stammende, von DE MAN als var. von hippothoë aufgefasste Form ist identisch mit dem von mir (Zool. Jahrb. V, IS9o, p. 474, pl. 36, fig. I2) von Tahiti beschriebenen A. acanthomerus.

Amboina, 2 Ex., Thursday Island, 2 Ex. (SEuor coll.).

Kilwa, am Strande, zwischen Stemen, I $\delta$ I t, Dar-es-Salaam, im Hafen bei Mtoni, in Spongien, I $\bar{t}$, Lpanga-Riff, in Korallkalk, I \& I $f$ (ORTs. coll.).

Verbreitung: Durch das ganze Indo-Pacifische Gebiet, vom Rothen Meer und Ost-Afrika bis Tahiti und zu den Sandwich-Inseln, ferner im tropischen Theil des Atlantic: Cap Verde-Insels und Bermuda, und Ostküste Amerikas von $\mathbf{N}$.-Carolina bis Brasilien. Auch an der Westküste Central-Amerikas (vgl. Miers).

Alpheus lobidens DE HAAN.

Vgl. Ontmaxi, Zool. Jahrb., T. 159(1, p. 474, pl. 36, fig. 13.

A mboina, 4 Ex. (SEMON coll.).

Kilwa, am Strande, zwischen Steinen, 2 Ex. (OrTMAN coll.).

$\mathrm{V}$ erbreitung: Bisher nur von Japan bekannt.

\section{Alpheus stremulls DAxA.}

Tgl. Ortuaxi, 1. c. p. 475.

Diese Form ist höchst wahrscheinlich nur als Tarietät ron A. lobidens aufzufassen. A. stremus (und wohl auch lubidens) soll nach Miers (Alert, I884, p. 286) das ¿ von $A$. edwardsi sein.

Thursday Island, 2 Ex. (SEmon coll.).

Verbreitung: Ton Ost-Afrika über Ceylon, den Malayischen Archipel bis nach Japan, den Sandwich-Inseln und Tongatabu.

1) Arch. f. Naturg., 53, 1, 1887, p. 518. Journ. Linn. Soc.. XXII, 188S, p. 268 pl. 17, fig. 1-5. Mergui-Ins., Java, Amboina. Ferner: Ceylon (Mus. Strassburg).

2) Arch. f. Naturg., 1887. p.5!8. 


\section{Alpllews villosus (OLIVIER).}

Mllaxe-Edwards, Hist. Nat. Crust., II, 1837, p. 354. Ricuter, Beitr. Moeresf., Maur. u. Seychell., 1889, p. 163. Haswell, Catal. Austr. Crust., 1882, p. 187. Miers, Rep. Zool. Coll. Alert, 1881, p. 290.

Diese seltene Art gehört in die Spinifrons-Gruppe DE MAN's (Arch. f. Nat. 53, I, I887, p. 498) und nach der von mir (1. c.) gegebenen Tabelle in die Abtheilung: A. BB. CC. D. Sie unterscheidet sich von allen übrigen Arten der Gattung durch den medianen Dorn im vorderen Theil des Cephalothorax hinter dem Rostrum und durch die Behaarung des ganzen Körpers.

Rostrum schlank, bis ans Ende des I. Stielgliedes der inneren Antennen, bei einem Exemplar sogar etwas darüber hinaus reichend, nach hinten bis zur Mitte des Cephalothorax als schwacher Kiel fortgesetzt. Dieser Kiel trägt im vorderen Theil des Cephalothorax dicht hinter den Augendecken einen scharfen Dorn. Augendecken mit je einem kurzen, scharfen Stachel.

Schuppe an der Basis der inneren Antennen mit dornartiger Spitze, fast so lang wie das I. Stielglied. Zweites Stielglied länger als das erste. Aeussere Antennen an der Basis mit einem langen, schlanken Stachel, der fast bis zum Ende des 2. Stielgliedes der inneren Antennen reicht. Schuppe schmal.

Erstes Beinpaar: grosser Scheerenfuss: Oberrand des Merus distal ausgezogen, innerer Unterrand distal mit einem kleinen Stachel. Hand comprimirt, fast oval, gegen die Basis breiter, nach den Fingern zu schmaler. Unter- und Oberrand ohne Kerben. Unterrand gerundet, Oberrand mit zwei fadenförmigen etwa parallelen Längsleisten, die eine Furche einschliessen. Aussentläche nahe der Basis des unbeweglichen Fingers mit zwei kurzen, über einander liegenden Längsdepressionen. Hand gegen die Finger etwas gedreht, so dass der bewegliche Finger in schräger Richtung articulirt. Oberrand dieses Fingers halbkreisförmig gekrümmt, der Finger viel kürzer als die Palma. - Kleiner Scheerenfuss: Merus wie beim grossen. Hand länglich, etwas comprimirt, Oberrand der Palma mit einer Längsleiste, distal an der Basis des beweglichen Fingers steht jederseits ein spitzer, vorwärts gerichteter Stachel.

Zweites Beinpaar: erstes Carpalglied das längste, etwa doppelt so lang wie das zweite. Das zweite nur wenig länger als das fünfte.

Merus des 3. und 4. Beinpaares distal am unteren Rande mit einem scharfen Dorn.

Der ganze Cephalothorax ist ziemlich dicht mit kurzen, steifen Haaren besetzt, dazwischen stehen längere (vielleicht ist aber die Behaarung allgemein länger gewesen und es sind die einzelnen Haare zun Theil abgebrochen. In ähnlicher Weise, aber weniger dicht ist das Abdomen behaart. Ebenso sind sämmtliche Beine behaart: am schwächsten ist die Behaarung auf der Aussenfläche der grossen Scheere, am stärksten auf deren Innenfläche und besonders auf dem Oberrand der grossen und kleinen Scheere und auf den Fingern.

Thursday Island, 3 Ex. (SEmon coll.).

Verbreitung: MrLne-Edwards giebt Australien an, und diese Angabe wird von Haswell wiederholt. Nach Richters bei Mauritius. Miers giebt Warrior Reef und Thursday Island an.

\section{Alpheus comatularum HASTELL}

Haswell, Catal. Austral. Crust., 1882, p. 189. Mrers, Rep. Zool. Coll. Alert, 1884, 1'. 28:.

Gehört in dieselbe Gruppe wie die vorhergehende Art.

Bei meinem o reicht das Rostrum bis über die Spitze des 2. Stielgliedes der inneren Antennen, beim $q$ ist es so lang wie der ganze Stiel. Beweglicher Finger der kleinen Hand länger als der unbeweg. liche, an der Spitze sichelförmig gebogen. Erstes Carpalglied des 2. Beinpaares so lang wie die übrigen vier zusammen, 2., 3. und 4. unter sich gleich, das 5. länger als jedes einzelne derselben. Merus des 3. und 4. Beinpaares distal am Unterrande mit einem spitzen Dorn. Beim o tragen die Epimeren des 2.-6. Abdomensegmentes, beim $q$ die des 2.-6. Abdomensegmentes kurze Stacheln. Ie Max.

Vgl. Miers, 1. c. und te Max (Arch. f. Naturg. 53, 1, 188\%, p. 508 ff.), leim Tergleiclı mit A. carinatus

Thursday Island, I $\delta$ I $f$ (SEnon coll.).

Verbreitung: Albany Passage, bei Cap lork, an Comatuliden (HAswelL), Torres-Strasse, an mehreren Orten, darunter Thursday Island (MIERs); ferner: Singapore und Ceylon (MiERs).

\section{Alphens bimmoiculatus STIMPSON.} A. biunguiculatus Strmpsox, Proceed. Acad. Nat. Sc. Philadelphia, 1860. p. 100. nE M.ax, Arch. f. Naturg., 53, 1, 1887,
p. 502, pl. 21, fig. 6.

A. tricuspidatus Helder, Sitz. Ber. Ak. Wiss. Wien, 44, 1861, 1. 267, pl. 3, fig. 15.

A. minor var. biunguiculatus DF MAs, Journ. Linn. Soc. London Zool., XXII, 188s, 1. 273.

Thu rsday Is land, I Ex. (SEMon coll.). 
Verbreitung: Rothes Meer (Helter); Ceylon (Mus. Strassburg); Mergui-Inseln (de Man); Java: Pulo Edam und Noordwachter (DE MAN); Sandwich-Inseln (STIMpsoN).

\section{Alpheus macrochirus RICHTERS.}

Togl. OkTmis. Zoul. Jahrb., V, 1890, p. 455.

Dar-es-S a la am, Upanga-Riff, in Korallen, $3 d 2+$ (ORTMANN coll.).

Verbreitung: Mauritius (Richters); Tahiti (Ortuanv).

\section{Alphews laevis RANDALL.}

Tgl. Okтмax, 1. c. p. 487 . Ferner: Mres, Alert, 1884, p. 561.

Amboina, I $d$ I 7 , zwischen fein rerzweigten, lebenden horallen (Pocillopora) (SEMON coll.).

Dar-es-Sala am, Chokirbank und Ras Rongoni, in Korallialk, 7 o 4 (OrTMANN coll.).

Verbreitung: Indo-Pacifisches Gebiet, vom Rothen Meer bis Japan, Sandwich-Inseln, Tahiti und Sydney. - Von Zanzibar durch Hilgexdorf und Pfeffer angeführt, ebenso bej den Amiranten und Seychellen nach MiERs.

\section{Alphens grocilipes Stinpsow}

Tgl. Ontuaxi, 1. с. 1. 488 .

Der A. gracilipes bei Thallwtz (Abh. Mus. Dresden, 3, I89!, p. 2I) scheint mir hiervon verschieden zu sein. Nach ThAllwitz sind die Augendecken gerundet, was nach der Beschreibung bei Stumpsov nicht der Fall ist. STupsox sagt: ,orbitae antice acutae, potius quam spiniferae", was für meine Exemplare vorzüglich passt. Ausserdem beschreibt THALLWiTz vor der Querfurche am Oberrand der Palma je einen ,zahnartigen, aufwärts gerichteten Fortsatz, von denen der äussere durch zwei dreieckige, eingedrückte Felder hegrenzt wird". lch finde eine derartige Angabe bei Stupsos nicht, und auch die mir vorliegenden Exemplare zeigen diese Bildung nicht. Das hier vorliegende Exemplar stimmt vollkommen mit den von mir von den Narquesas- und Samoa-Inseln erwähnten (1. c.) überein. Ob A. gracilipes bei Miers (Alert, 188 , p. 28 7 ) hierher gehört, ist zweifelhaft.

Dar-es-Sala a m, Upanga-Riff, in Korallen, i d (ORTMANN coll.).

Verbreit ung: Von Ost-Afrika bis Japan, Tahiti und zur Bass-Strasse. - Von Zanzibar durch PFEFFER angegeben.

\section{Alphens fiontrulis SAY.}

Vgl. Ortmaxi, 1. e. 1890, p. 488.

Identisch hiermit ist ferner:

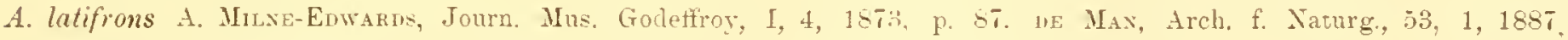
1. 521 , pl. 22 , fig 4.

Betaeus utricola Richtens, Beitr. Meeresf. Maur. Seych., 1880, p. 164, pl. 17, fig. 34, 35.

A. Milve-Edwards sagt zwar ausdrücklich, dass sein latifrons verschieden sei von frontalis: jedoch konnte nach DE MAX zwischen dem Original des latifrons und einem Exemplar DE MAN's von Amboina von PFEFFER kein Unterschied gefunden werden. Diese Exemplare DE MAN's stimmen nun aber wiederum völlig mit meinen Exemplaren, die ich 1. c. als frontalis anführte, ebenso wie mit der Abbildung des frontalis des älteren Milne-Edwards, im Atlas zu Cuvier's règne animal, Crust. pl. 53, fig. 2. Der latifrons muss also mit frontalis identisch sein.

A mboina, 9 Ex., aber kein erwachsenes of darunter (SEnox coll.).

Verbreitung: Samoa-Inseln (A. Milve-Edwards, Ortanns); Tahiti (Heller); Australien (MilveEdwards); Liu-Kiu-Inseln (ORTuANe); Amboina (DE MAN). Richters giebt keinen Fundort an, wahrscheinlich ist es Mauritius.

\section{Familie: Hippolytidae.}

\section{Shron masumorntus (OLIVIER).}

Vgl. Hippolyte marmorata OL., ORтмass, Zool. Jahrb., T, 1890, p. 497.

Hiermit ist wohl auch identisch die H. hemprichi Heller. Meine Bemerkung (1. c. p. 498) über ihre Verschiedenheit beruht auf einem Irrthum. 
Die von Thallwitz (Zool. Anzeig., I891, p. 99, und Abhandl. Mus. Dresden, I89I, p. 25) aufgestellte Gattung Saron wird sich wohl halten lassen.

Meine $\delta$ zeigen nicht die enorm entwickelten dritten Maxillarfüsse: dieselben sind aber auch kaum halb so gross als die mir aus der Südsee vorliegenden $\}$, welche dieses für marmoratus als charakteristisch angeführte Merkmal zeigen. Die Dornen an dem Merus der 3., 4. und 5. Pereiopoden entsprechen dem Verhalten bei S. marmoratus, nicht dem bei gibberosus.

Dar-es-S a la am, Upanga-Riff, zwischen Korallen, $2 \delta 4 q$ (OrTMañ coll.).

Verbreitung: Bisher sind nur die Sandwich-Inseln und Amboina als sichere Fundorte bekannt. Von Ost-Afrika wurde diese Art (wie auch der nahe verwandte S. gibberosus) noch nicht angeführt. Dagegen scheint sie im Rothen Meer vorzukommen (H. hemprichi Helder, vgl. auch DE MAx. Not. Leyd. Mus., III, I88 I, p. IO7).

\section{Familie: Pontoniidae.}

Pontonia pinuae nov. spec. Tafel l, Fig. 3.

Cephalothorax länger als breit (in der oberen Ansicht), gleichmässig gewölbt, weder comprimirt noch deprimirt. Rostrum etwas abwärts geneigt, oben breit, ohne Kiel, stumpf, bis zur Mitte des 2. Stielgliedes der inneren Antennen reichend. Endfäden der inneren Antennen von der typischen Gestalt. Die der äusseren kaum länger als der Cephalothorax. Schuppe oval, am inneren und vorderen Rande behaart.

Merus und Carpus der ersten Pereiopoden gleich lang. Zweite Pereiopoden sehr ungleich, der grössere fast so lang wie der Körper. Scheere desselben länger als der Cephalothorax, fast cylindrisch, ohne Kanten. Finger viel kürzer als die Palma, der bewegliche an der Basis mit einem Höcker, der in eine Grube des unbeweglichen greift, letzterer ohne Zähne. Die hinteren Pereiopoden sind gleichmässig, mit schwachen, einfachen, gekrümmten Klauen.

Abdomen nicht comprimirt. Telson lang-dreieckig, mit abgerundeter Spitze, daselbst mit 6 kurzen Dörnchen.

Unterscheidet sich von P. tridacnae (РET.) und P. maleagrinae (PET.) durch die ungleichen Scheeren und den schlankeren Körper. Von tridacnae noch durch einfache Klauen und von maleagrinae durch den Mangel der Zähne des unbeweglichen Scheerenfingers. In der Körpergestalt und durch die ungleichen Scheeren nähert sich diese Art den Mittelmeerarten, unterscheidet sich aber durch stumpfes Rostrum, einfache Klauen und die Bezahnung der Scheerenfinger. P. flavomaculata HELL. hat ferner eine scharfrandige Palma der Scheeren, P. tyrrhena Riss. stumpf kantige Ränder der Palna.

Dar-es-Salaam, Chokirbank, in den Schalen einer Pinna-Art lebend, und zwar je $1 \delta$ und I 1.

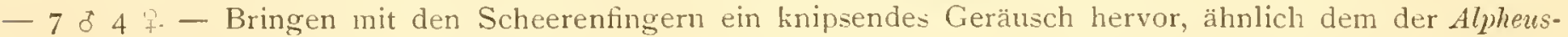
Arten (Ortmann coll.).

\section{Corrlliocaris gruminea (DANA).}

Qedipus gramineus Daxa, U. S. Expl. Exp. Crust., 1852, p. 5T4, pl. 37. fig. 3. Pfeffer, Jahrb. Hamb. Wiss. Aust., II, 1889 , p. 34 .

Coralliocaris graminea (Dax.) Stimpsox, Proc. Acad. Nat. Sc. Philadelphia, 1860, p. 107. Mriers, Rep. Coll. Alert. 1884. p. 563. DE MAx, Arch. f. Naturg., 53, 1, 1887, p. 536

Dar-es-S a la am, Upanga-Riff, zwischen Korallen, 2 I $q$ (ORTMANN coll.).

Verbreitung: Zanzibar: Changu-Riff (Pfeffer); Seychellen (Miers); Java: Pulo Edan (DE MAN ; Hongkong (Stimpson); Fidji-Inseln (DANA, DE MAN).

\section{Anchistia cusifroms DANA.}

Dana, U. S. Expl. Exp. Crust., 1852, p. 5\$0, pl. 38, Fig. 1. Müluer, Terh. Naturf. Gesellsch. Basel, 8, 2. 1887, p. $4 \overline{1} 1$. Das $q$ mit DANA's Beschreibung völlig übereinstimmend, beim $\delta$ ist das 2. Beinpaar kräftiger, Merus und Carpus desselben unten distal mit je einem feinen Dorn. Unterrand des Rostrums beim $\delta$ mit 4 , beim 9 mit 2 Zähnen.

Dar-es-S a l a m, Upanga-Riff, zwischen Korallen, I J, am Wachthaus am Hafeneingang, zwischen Korallen-Gerölle, i $\subsetneq$ (ORTMANN coll.).

Verbreitung: Bisher nur ron Nord-Borneo: Balabac-Strasse (DANA) und von Cerlon: Trincomali (MüLLER) angegeben. 
Familie: If ymenoceridae.

Hymenocerr elegars HELLER.

Vgl. Ortmaxi, Zool. Jahrb., T, 1890, 1. 511.

Amboina, I Ex. (SEMON coll.).

Verbreitung: Bisher nur aus dem Indischen Ocean bekannt. Rothes Meer: Tur am Sinai

(Heller); Mozambique (Hilgendorf); Mauritius (OrtmanN).

\section{Familie: Palaemonidae.}

\section{Leander lomgicammes STIMPSON.}

Vgl. Ortmasi, Zool. Jahrb., T, 1890. p. 516.

Bezahnung des Rostrums im Mittel $\frac{6+1}{4-5}$, selten mehr oder weniger Zähne. Höchste Zahl oben:

$7+1$, niedrigste: $5+1$, höchste unten: 7 , niedrigste: 0 .

Carpus des 2. Beinpaares die Spitze der Antennenschuppe erreichend, länger als die Scheere.

Dar-es-Sala am, im Hafen bei der evangelischen Mission, in Wasserlöchern, die bei Ebbe zurückbleiben. Zahlreiche Exemplare. Ferner wurden mir viele Exemplare zusammen mit Penaeus, Caridina etc. gebracht, die vielleicht von der Upanga-Mündung stammen.

Verbreitung: Zanzibar (Hulgexdorf); Hongkong (Stripson); Amboina (de MaN); MarshallInseln (ORTMANN).

\section{Palaemon lar FaBRICIUS.}

Vgl. Pal. mber Hess. Ortmaxi, Zool. Jahrb., V, 1891, p. 705.

Pal. lar Fabr. Ortalas, ibid. p. 724. de Mas, in: Weber, Zool. Erg. Reis. Nied. Ost-Indien, II, 1892, p. 445.

DE MAN (1. c. p. 410) bezweifelt die Brauchbarkeit der Gestalt der Telsonspitze zur Unterscheidung gewisser Palaemon-Arten. Ich muss ihm jetzt hierin Recht geben, zunächst was den $P$. ruber und lar anbelangt. Dagegen halte ich die von mir angegebene Eigenthümlichkeit der Telsonspitze jedenfalls für P. lamarrei aufrecht, von dem ich nunmehr über hundert Exemplare in Händen hatte, die ich stets an dem angegebenen Merkmale erkannte. Auch für $P$. jamaicensis und vollenhoveni, die ich jetzt übrigens für identisch halte, scheint mir das breite Ende des Telsons charakteristisch zu sein.

Wo ich sonst (l. c.) dieses Merkmal angewandt liabe, mag ebenfalls die Ansicht DE MAN's ihre Richtigkeit haben.

Amboina, Süsswasser, viele Ex. Neu-Guinea, Süsswasser, 4 Ex. (Semon coll.).

Ganz allgemein im Indo-Pacifischen Gebiet verbreitet: von Madagascar und den Mascarenen über die Ost-Asiatische Inselwelt bis zu den Philippinen, Australien, Fidji und Tahiti. - Von Amboina und Neu-Guinea schon bekannt.

\section{Pralremon australis ORTMANN.}

Palaemon nov. spec.? IDE MAN, Zool. Jahrb., II, 1887, p. 711.

Palaemon spec.? (P. nustralis) Ortians, ibid., V, 1891, p. 708.

Diese Art gehört in die allernächste Verwandtschaft von P. lar. Mir liegt eine Anzahl erwachsener § vor, bei denen die Scheerenfüsse folgende Verhältnisse zeigen.

Merus, Carpus und Palma ungefähr von gleicher Länge (die Unterschiede betragen höchstens einige Millimeter, z. B.: Merus: 20, Carpus: 22, Palma: 23,5, Finger: 18). Die Finger sind kürzer als die Palma (in der Jugend oft ebenso lang). Die Palma ist sehr schwach comprimirt. Charakteristisch für die Art ist die dichtfilzige Behaarung der Finger, während sonst das ganze Bein fein stachelig erscheint. Beide Finger besitzen auf den Schneiden nahe der Basis je zwei Zähne. Das Rostrum ist ziemlich variabel, über den Augen leicht convex, sonst fast gerade vorgestreckt oder seltener an der Spitze unbedeutend aufgebogen. Es überragt niemals die Antennenschuppen, endigt meist zwischen dem Ende der inneren Antennenstiele und der Spitze der Antennenschuppe. Die Zähne sind: $\frac{7-12}{3-6}$, von den Zähnen des Oberrandes stehen zwei oder drei auf dem Cephalothorax.

Ob $P$. danae HELler von Sydney hierher zu rechnen ist, würde nur dann entschieden werden können, wenn bei Sydney keine andere Palaemon-Art vorkommt. Vorläufig ist P. danae nicht identificirbar. 
Queensland, Burnett, viele alte und junge Exemplare (SEMON coll.).

Terbreitung: Bisher auf das nordöstliche und östliche Australien beschränkt. Queensland: Gayndah, Rockhampton, Peak Downs (Ortians). Sydney (de Max).

\section{Pataemon eleyans DE MAN.}

de Max in: Weber. Zool. Erg. Reis. Niederl. Ost-Ind., II, 1892, p. 440, pl. 26, Fig. 36.

Buitenzorg, 2 Ex. (SEmon coll.).

Verbreitung: Java: Buitenzorg und Sinagar (DE MAN).

\section{Palaemon cf: idae Heller.}

Vgl. Ortmaxx. l. c. p. 717 .

Mir liegt nur ein jüngeres $q$ vor, dessen Scheeren noch nicht tỵpisch entwickelt sind. Jedenfalls ist der Carpus bedeutend länger als die ganze Scheere, und das Exemplar gehört demnach in die Verwandtschaft von nipponensis, lanceifrons, idae und dispar. Nach der Gestalt des Rostrums würde es am besten mit $P$. idae passen, der ja auch von Zanzibar bekannt ist. Mein Exemplar hat oben 8, unten 3 Zähne, am Oberrand scheint jedoch der vorderste unterdrückt zu sein, da der Platz für ihn vorhanden ist.

Dar-es-Salaam, wurde mir von Negern gebracht, wohl Upanga-Nündung.

Verbreitung: Zanzibar (HıgENDorf); Seychellen (Richters); Mauritius (Richters); Singapur (v. Martens); Java (v. Martexs); Borneo (Heller); Philippinen (v. Marters).

\section{Palcemon disprer v. MARTENS.}

Tgl. Ortmans, l. c. p. 718. Thallittz, Abh. Mus. Dresden, 3, 1891, p. 15. De Mas, in: Weber etc., 1892, p. 427, pl. 20. Fig. 34 .

Amboina, Süsswasser, 2 Ex. (SEmon coll.).

Verbreitung: Mascarenen (Hoffuann, Ruchters, Miers); Sunda-Inseln: Samangkabaai, Tandjong (Miers), Celebes (Thallwitz, DE MaN), Amboina (DE Man), Flores (DE Man), Adenare bei Flores (r. Martens, de Man), Timor (de Man), lns. Saleyer (de MaN); Samoa-Ins. (Miers).

Eine Anzahl weiterer Exemplare, zur Gattung Palaemon gehörig, von Dar-es-Salaam und Amboina lassen sich wegen ihres allzu jugendlichen Zustandes nicht bestimmen.

\section{Familie: Stenopilate.}

\section{Stenopus hispidus (OLIVER).}

Milie-Edwarus, H. Nat. Crust., II. 1837, pl. 25, fig. 13, Adaus et White, Zool. Toy. Samarang Crust., 1848, p. 61, pl. 12. fig. 6, Daxa. U. S. Expl. Exp., 1852, p. 607, pl. 40, fig. 8, Richters, Beitr. Meeresf. Maur. Sexch., 1880, p. 166. DE Max, Arch. f. Naturg., 53, 1, 1887, p. 566. Ortmaxi, Zool. Jabrb., V, 1890, p. 539.

Ob die im Atlantic vorkommende Form (Cuba, Bahama, Bermuda) mit der Indo-Pacifischen identisch ist, bleibt noch zu untersuclien.

Tgl. v. Martexs, Arch. f. Naturg. 38. 1, 1872, p. 143. Bate, Chall. Macrur., 1888, p. 211. pl. 30. Brooks et Herrick, Nation. Acad. Scienc., V, 4, 1892, p. 348, pl. 5.

A mboina: 12 Exemplare.

Verbreitung: Indischer Ocean (Milne-Edwards); Mauritius und Seychellen (Richters); Ceylon (Müller); China-See (Adams et WWhite); Philippinen (Adams et White); Borneo (Adams et White); Balabac-Strasse (Dana); Amboina (DE Man, Ortuann); Neu-Guinea (Miers); Paumotu-Inseln (Dañ).

\section{Stenomus tennirostris DE MAN.}

DE Max, Arch. f. Naturg., 53, 1, 1887, p. 567, pl. 22a, fig. 5.

A m boina: 3 Ex. (SEMON coll.).

Verbreitung: Amboina (DE MAN). 
Pamulimens molyuhagus (Herbst) I796.

Cancer Astacus) polyphagus Hersst, Krabben und Frebse, II, 1796, p. 90, pl. 32.

Palinurus ornatus Fanricios, Suppl. Entomol. Syst., 1798, p. 400.

Palinurus polyphagus Hвst Latrenlle, Annal. MIus. Hist. Nat., Paris, III, 1804, p. 393.

Palinumes versicolor Latreille, ibid, p. 394.

Der Speciesname polyphagus hat, wie aus obigen Citaten hervorgeht, die Priorität. Die übrige Literatur siehe unter Senex ornatus (FAbr.) bei Ortmaxi, Zool. Jahrb., VI, I, iSgi, p. 34.

Die jungen Exemplare von Amboina und Neu-Guinea sind äusserst interessant, weil an ihnen sämmtliche Merkmale der Art vollkommen ausgebildet sind: der dritte Maxillarfuss besitzt keine Exopoditen, die Abdomensegmente zeigen keine Querfurchen und das Antennensegment trägt vier Dornen. Sie besitzen ausserdem eine charakteristische Zeichnung: die Grundfarbe des Körpers ist ein helleres oder dunkleres Braun, der Cephalothorax hat je einen seitlichen weissen Längsstreifen und je einen schrägen weissen Streifen, der von der Mitte des Cephalothorax (dicht linter der Cervicalfurche) nach hinten und aussen zum hinteren Ende des Seitenstreifens läuft. Die weissen Streifen bilden also eine W-förmige Figur. Die Maasse dieser jungen Exemplare sind folgende: von Amboina: I) Totallänge: 26 mm, Cephalothorax: 9 mm; 2) Total: 22.5 mm, Cephalothorax $7.5 \mathrm{~mm}$; 3) Cephalothorax $9 \mathrm{~mm}$, die Totallänge lässt sich nicht gut messen, da das Abdomen etwas verdriickt ist. Von Neu-Guinea zeigt ein Exemplar total: $24 \mathrm{~mm}$, Cephalothorax Io mm, das andere Exemplar ist grösser, aber bei einer Cephalothoraxlänge von schon I9 mn zeigt es noch dieselbe eigenthümliche Färbung. Zwei grössere Exemplare von Amboina von $26,5 \mathrm{~mm}$ und $34 \mathrm{~mm}$ Cephalothoraxlänge zeigen diese Jugendfärbung nicht mehr. Beide Exemplare sind q, zeigen aber noch keine Scheerenbildung an den 5. Pereiopoden. Das Exemplar von der Thursday-Insel ist das grösste, ein $\delta$ mit $53 \mathrm{~mm}$ Cephalothoraxlänge.

Amboina, 2 mittlere 7,3 juv. Neu-Guinea, 2 juv., Thursday Island, I 8 (SEMON coll.). Verbreitung: Von Ost-Afrika bis zu den Samoa-Inseln bekannt.

\section{Purr spiniger nox. spec. Tafel II, Fig. 2.}

Diese neue Art stimut vollkommen in der äusseren Körpergestalt und der Bedornung des Cephalothorax mit dem ron mif (Zool. Jahrb., VI, I, IS9I, p. 37, pl. I, fig. 3) beschriebenen P. pellucidus von Japan überein, wobei zu bemerken ist, dass bei beiden Arten dicht hinter den Augenhörnern noch je ein feines Dörnchen steht, das ich seiner Zeit übersehen hatte.

$P$. spiniger unterscheidet sich aber ron $P$. pellucilus durch den Mangel des Exopoditen am dritten Maxillarfuss, der bei letzterer Art als kurzes, zweigliedriges Stück (vergl. 1. c. pl. fig. 3i) verhanden ist. Ferner verlängert sich bei $P$. spiniger das Sternum jederseits an der Basis der 5. Pereiopoden nach hinten in einen spitzen Dorn. Dieser Dorn fehlt bei der anderen Art.

Man könnte auf den Gedanken kommen, dass die Arten dieser Gattung als Jugendformen zur Gattung Pamlirus zu zielıen sind. Jedoch die oben erwähnten jugendlichen Exemplare von Pamulirus polyphagus, die den vorliegenden Exemplaren und denen des Puer pellucidus in der Körpergrösse gleich stehen, machen mir jetzt dieses Verhälniss unwahrscheinlich, da jene schon alle zur specifischen Unterscheidung der Pamulirus-Arten nöthigen Merkmale erkennen lassen, und selbst in untergeordneten Charakteren, wie Bedornung des Cephalothorax, erwachsenen Exemplaren ähneln. Die Gattung Puer weicht dagegen besonders in der Gestaltung und Bewehrung des Cephalothorax, des Antennensegmentes so wesentlich von allen Arten der Gattung Panulirus ab, dass sie mit dieser nicht in Beziehung gesetzt werden kann. Ob sie dagegen ïberhaupt als erwachsene Formen anzusehen sind, oder als Jugendformen irgend einer anderen unbekannten Palinuriden-Form, bleibt vorläufig noch dahingestellt.

Amboina, 4 Ex. (SEMON coll.).

\section{Familie: Scyllaridae.}

\section{Gattung: Scyllarms.}

Den Sc. haani habe ich (Zool. Jahrb., VI, I, ISgI, p. 40) irrthümlicher Weise für identisch mit Sc. sieboldi erklärt, da mir kein echter haani vorlag. Um das gegenseitige Verhältniss der bekannten Scyllarus-Arten klarzulegen, mag folgende Tabelle von Nutzen sein.

a. Carpus der ersten und zweiten Pereiopoden oben mit je zwei scharfen Kielen, ron denen besonders der vordere der ersten Pereiopoden oft flügelartig erhaben ist. Auch die Meren und Carpen der 
übrigen Beine mit ähnlichen flügelartigen Kielen. Cephalothorax und Abdomen nur schwach buckelig.

aa. Carpus der ersten und zweiten Pereiopoden oben ohne Kiele, höchstens nur mit ein bis zwei undeutlichen, stumpfen Wülsten.

b. Kiele auf den Meren der I.-4. Pereiopoden, sowie der vordere Kiel auf den Carpen der dritten und vierten Pereiopoden stark flügelartig erhaben (etwa $\frac{1}{3}$ der Breite des betreffenden Gliedes betragend). Propodus des zweiten Pereiopoden oben mit deutlicher Kante.

c. Abdomen schwach buckelig. Zweites freies Glied der äusseren Antennen an der vorderen äusseren Ecke mit hakenförmig nach oben gekrümmtem Dorn. Sc. latus LATr. ${ }^{2}$ )

cc. Abdomen auf dem 2., 3. und besonders 4. Segment stark buckelig erhaben. Zweites freies Glied der äusseren Antennen mit geradem, nicht hakenförmigem Dorn.

bb. Kiele der Meren nicht auffällig flügelartig erhaben, ebenso die der Carpen. Zweites Glied der äusseren Antennen mit geradem, nicht hakenförmigem Dorn. Propodus der 2. Pereiopoden völlig gerundet, ohne Kante.

c. Abdomensegmente nicht auffällig buckelig erhaben. Cephalothorax hinter den Augen seitlich fast ganz ohne Kerbe.

Sc. aequinoctialis FABR. ${ }^{3}$ ) und herklotsi HERKL. ${ }^{4}$ )

cc. Abdomensegmente buckelig erhaben, besonders der 3. und 4. Cephalothorax hinter den Augen seitlich mit je einer deutlichen Kerbe.

Sc. elisabethae nov. spec.

Scyllarus haani DE HAAN.

de HaAn, Faun. japonic. dec., 5, 1841, p. 152, pl. 38, fig. 1. Miers, Ann. Mag. Nat. Hist. (5), V, 1880, p. 377. de Man, Arch. f. Naturg., 53, 1. 1887, p. 485.

Amboina, i $q$ (SEmon coll.).

Verbreitung: Japan (DE HAAN); Amboina (DE MAN); Aru-Insel (MIErs).

\section{Scyllarus elisabethae nox: spec. Taf. II, Fig. 3.}

Diese Art unterscheidet sich von allen übrigen schon im Habitus, dessen Eigenthümlichkeit durch die scharfe Kerbe des Seitenrandes bedingt wird.

Der Cephalothorax ist mit dichtstehenden rundlichen Körnern bedeckt, wie bei den übrigen Arten, doch vermisse ich bei dem vorliegenden Exemplar die sonst in den Furchen stehenden Haare, die jedoch, da das Exemplar trocken conservirt ist, abgerieben sein liönnen. Die Buckel des Cephalothorax sind ziemlich deutlich ausgebildet, stärker als bei Sc. aequinoctialis. Das Abdomen zeigt auf dem 2. bis 5. Segment in der Mitte je einen Buckel, der besonders auf dem 3. und 4. auffällt, jedoch sind diese Buckel nicht so auffallend wie bei Sc. haani. Die Sculptur des Abdomen ist ähnlich der des Cephalothorax. Die Epimeren des 2. Abdomensegmentes zeigen, im Gegensatz zu allen übrigen Arten, am Vorderrand keine Zähne.

Auch die äusseren Antennen weichen in der Gestalt des zweiten freien Gliedes nicht unwesentlich von den übrigen Arten ab: der Aussenrand bildet nahe der Basis nicht die scharfe, fast rechtwinklige Umbiegung, die sich bei den anderen Arten beobachten lässt, sondern verläuft von der Insertionsstelle genau in einem Halbkreis nach aussen und vorn, um erst dann in flacherem Bogen zur Spitze zu ziehen. Die Länge dieses Gliedes, von der Mitte der Insertion am ersten freien Gliede bis zur äusseren vorderen Spitze gemessen, ist deshalb etwas so gross wie seine Breite nahe der Basis, von der Spitze des Dornes am Innenrande zur der Stelle des Aussenrandes gemessen, die der äusseren Vorderecke des Cephalothorax gegenüberliegt. Bei den übrigen Arten ist die Breite deutlich beträchtlicher als die Länge.

Die Kiele auf den Pereiopoden sind bei dieser Art am schwächsten von allen entwickelt. Man kann nur auf den Meren, sowie den Carpen des 3. und 4. Beinpaares solche unterscheiden. Die Meren und Carpen sind theils mit Körnern wie der Cephalothorax, theils mit grubigen Vertiefungen besetzt, eine Sculptur, die sich in dieser Weise bei keiner der anderen Arten findet.

Capland: Port Elisabeth, I d (im Mus. Strassburg).

1) DE HaAn, Faun. japon. dec. 5, 1841. p. 152, pl. 36 und 37, fig. 1. Stinpson, Proceed. Acad. Nat. Sc. Philadelphia, 1860, p. 92. Ortmann, 1. c. 1891, p. 40, z. T. Japan, Lin-Kin Inseln. Ferner Mauritius (Mus. Strassburg). Vielleicht identisch mit Sc. squamosus M -E. (Hist. Nat. Crust., II, 1837. p. 284).

2) Vgl. Ortmann, 1. c. 1891 , p. 38. - Mittelmeer. Canarische Inseln.

3) Vgl. Ortmann, ibid. p. 39. - West-Indien und Brasilien.

4) Herklots, Addit. faun. carcin. Afric. occid., 1851, p. I4, pl. 2. - West-Afrika. 
Familie: Honaridae.

Enoplometopus pietus A. MiLne-Edwards.

- Vgl. Miers, Ann. Mag. Nat. Hist. (5), T, 1880, p. 380. we MAx, Arch. f. Naturg., 53, 1, 1887, p. 486, pl. 21, fig. 3.

Miers (1. c.) glaubt gesehen zu haben, dass bei dieser Art am 2. Maxillarfuss der Epipodit (Mastigobranchie) fehlt, während die Kieme (Podobranchie) entwickelt sei: ich kann diesen Befund $\mathrm{n}$ ich $\mathrm{t}$ bestätigen. Sowohl am 2. als auch am 3. Maxillarfuss ist Kieme und Epipodit in der gewöhnlichen Weise entwickelt, die erstere nur wenig kürzer als der letztere. Am 2. Maxillarfuss sind beide Theile schwächer als am dritten, wie grewöhnlich. Es würde dieses Merkmal die Gattung Enoplometopus näher zu Homarus bringen und von Nephrops trennen, vorausgesetzt, dass das Fehlen der Podobranchie am 2. Maxillarfuss bei den Arten von Nephrops allgemein vorkommt.

Amboina, 2 Ex. (Semon coll.).

Verbreitung: Réunion (A. Milne-Edwards); Amboina (Miers, de Man).

Familie: Astacidae.

Cheraps bicarinatus (GRAY).

Vgl. Ortmaxy, Zool. Jahrb., VI, 1, 1891, p. 7.

$Q$ u e ensland, Burnett, 8 Ex. (Semon coll.).

Verbreitung: Port Essington (Gray); Cap York (v. Martens); Queensland: Rockhampton (OrT. MANN); Murrayfluss (v. Martens).

Familie: Thalassinidae.

Thalassina anomala (Herbst).

Tgl. Ortmanx, 1. c. p. 52. Thallwitz, Abh. Mus. Dresden, 1891, p. 30.

A mboina, 2 Ex. (Semon coll.).

Verbreitung: Von Ceylon über den malayischen Archipel und die Pacifischen Inseln angeblich bis Chile. - Den von mir 1. c. angeführten Fundorten sind folgende hinzuzufügen: Borneo, N.-W.-Australien und Thursday Isl. (Miers); Celebes, Mysore, Amboina, Sumba (Thallwitz). Ferner sah ich Exemplare, die Herr Dr. Fritze von den Liu-Kiu-Inseln mitbrachte. Letztere sollen ihm „aus dem Walde“ gebracht sein. Nach Mlers (Alert, I884, p. 283) lebt die Art in Mangrove-Sümpfen.

\section{Familie: Callianassidae.}

\section{Gattung: Gebia.}

Ueber die Arten der Gattung Gebia herrscht noch sehr viel Unklarheit. Ich will hier versuchen, eine Anzahl derselben tabellarisch zusammenzustellen.

a. Palma der Scheeren an der Innenseite, nahe dem Unterrande, kurz vor der Basis des unbeweglichen Fingers oline Dorn.

b. Carpus der Scheerenfüsse am Unterrande mit einem Dorn. Merus der I. und 2. Pereiopoden am distalen Ende des Oberrandes mit einem Dorn.

c. Carpus oben distal mit einem Dorn.

G. litoralis (RIss) ${ }^{1}$ ).

cc. Carpus oben mit einem Dorn, ferner mit einem Dorn dicht daneben an der Innenseite. Auf der Aussenseite nahe dem Unterrande eine Reihe Dörnchen.

bb. Carpus der Scheerenfüsse am Unterrande ohne Dorn.

G. major D. H. ${ }^{2}$ ).

aa. Palma der Scheeren ebenda mit einem Dorn.

b. Merus der I. und 2. Pereiopoden am Oberrande ohne Dorn.

G. hirtifrons $\mathrm{WHITE}^{3}$ ).

G. africana n. spec.

bb. Merus der I. und 2. Pereiopoden am Oberrande distal mit einem Dorn. Telson mit einer scharfen Querleiste nahe dem vorderen Rande.

c. Ischium des I. Beinpaares, und Basis des 2. und 3. Beinpaares ohne Dornen.

G. barbata STRAHL.

1) Vgl. ORtmanM, Zool. Jahrb., VI, 1, 1891, p. 53. - Europa.

2) Vgl. OrtManN, ibid. p. 54. - Japan.

3) Vgl. Haswell, Catal. Austral. Crust. 1882, p. 164. - Australien. 
cc. Ischium des I. Beinpaares mit 2 Dornen, Basis des 2. und 3. Beinpaares mit je einem kurzen Dorn.

G. carinicauda STPS. ${ }^{1}$.

Gebia aficana nox. sper. Tafel II, Fig. 4.

Stirn wie gewöhnlich mit zwei seitlichen Furchen, deren jede aussen durch eine Körnerreihe begrenzt wird, die nach vorn mit einem spitzen Zahn vorspringt. Der dazwischen liegende Theil ist etwa oval, vorspringend, spitzlich, in der Mitte mit einer Längsfurche, seitlich von dieser Furche stehen zwei Körnerreihen, die äussere Reihe verläuft am freien Rande des mittleren Stirnfortsatzes und zwar stehen an dem freien Rande selbst vor der seithichen Kerbe je 4-5 Körner, die kurz dornförmig nach oben gerichtet sind. Das vorderste Dörnchen jederseits ist von den anderen etwas abgerückt.

Stiele der inneren Antennen kürzer als die der äusseren, bis zur Spitze des vorletzten Gliedes der letzteren reichend.

Merus des I. und 2. Beimpaares oben unbewehrt. Merus des I. Beinpaares am Unterrande mit einer Reihe etwas ungleicher Dörnchen. Carpus des I. Beinpaares oben mit einem kürzeren, unten mit einem längeren Dorn, seine Aussenseite nahe dem Unterrande mit einem glatten Kiel, der nach vorn in ein kurzes Dörnchen endigt. Palma der Scheere an der Innenseite, nahe dem Unterrande und nahe der Basis des unbeweglichen Fingers mit einem Dorn. Ebenda steht eine Anzahl Körner, von denen eines, dicht an der Basis des beweglichen Fingers, dornförmig ist. Diesem gegenüber steht auf der Aussenseite der Palma, dicht an der Basis des beweglichen Fingers, ein ähnliches Dörnchen. Auch nahe dem Unterrande der Palma stelien auf der Aussenseite einige spitze Körner und Dörnchen. Beweglicher Finger über doppelt so lang als der unbewegliche, am Oberrand und auf der Aussenfläche desselben verläuft je eine Reihe spitzer Körner.

Ischium des I. Beinpaares mit einem oder zwei kleinen spitzen Körnern. Die Basen der folgenden Beinpaare sind unbewehrt.

Das Telson fehlt bei meinem Exemplare.

Der Fundort dieser Form legt es nahe, sie mit den beiden schon vom Cap bekannten Arten zu vergleichen. Leider ist die G. major var. capensis bei Krauss (Südafrik. Crustac., I843, p. 54) viel zu unvollständig beschrieben. Die G. subspinosa bei Strmpson (Proceed. Acad. Nat. Sc. Philadelphia, I860, p. 9I) scheint ihr nahe zu stehen. Aber Stimpson beschreibt auf der Innenseite der Hand zwei Dornen an der Basis des Dactylus und ferner sollen die Beine des I., 2. und 3. Paares an der Basis einen spitzen Dorn zeigen.

Port Elisabeth, ein sehr verletztes, trocken conservirtes Exemplar (im Mus. Strassburg).

Gebia barbata STRAHL.

? G. barbata Strahl, Mon. Ber. Akad. Wiss. Berlin, 1861, p. 1062, fig. 7-9.

G. barbata Orthans, Zool. Jahrb., TI, 1, 1891, p. 54, pl. 1, fig. S.

Ein mir von Amboina vorliegendes Exemplar stimmt vollkommen mit den seiner Zeit von mir erwähnten Exemplaren von den Samoa-Inseln. Es steht diese Form der G. carinicauda (l. 1. c. c.) sehr nahe, unterscheidet sich aber sofort durch den Mangel der Dornen an der Basis der 2. und 3. Pereiopoden; auch am Ischium der I. Pereiopoden steht kein Dorn, sondern nur ein Körnchen.

Mittlerer Theil der Stirn (zwischen den Seitenfurchen) verhältnissmässig wenig vorspringend, ohne mittlere Längsfurche, mit 4 Dörnchen.

Merus der I. und 2. Pereiopoden am Oberrande distal mit einem Dörnchen. Ischium der I. Pereiopoden unten mit einem kleinen Körnchen, das bisweilen ganz undeutlich ist. Merus am Unterrande gezähnelt. Carpus am Oberrande mit einen kleinen Dörnchen, am Unterrande unbewehrt. Palma mit einem Dorn auf der Innenseite nahe dem Unterrande, sonst ohne Dornen.

Zweites und drittes Beinpaar an der Basis unbewehrt. Telson mit einem Querkiel nahe dem vorderen Rande.

Ob diese Art mit der G. barbata STRAHL wirklich identisch ist, lässt sich wegen der mangelhaften Beschreibung der letzteren nicht entscheiden. Es würde wohl am besten sein, die barbata STRAHL als „nomen nudum" anzusehen.

Amboina, I Ex. (Senion coll.).

Verbreitung: Samoa-Inseln (Ortmann); Strahl giebt die Philippinen an.

1) Stimpson, Proceed. Acad. Nat. Sc., Philadelphia, 1860, p. 9I. Miers, Rep. Zool. Coll. Alert, 1884. p. 2S0. DE MAN, lourn. Linn. Soc. Zool., 22, 1858, p. 256. - Mergui-Ins., China, Torres-Strasse. 
Untergattung: Gebiopsis.

Tgl. Ortuaxx, Ergebn. Plankton-Exp. Decap. Schizop., 1893, p. õo.

Gebia (Gebiopsis) intermedia DE MAN.

DE Max. Arch. f. Naturg. 53. 1. 1887, p. 462 (rar.). de Max. Journ. Iinn. Soc. Zool., 22, 1888, p. 256, pl. 16, fig. 6-8. Die vorliegenden Exemplare stimmen mit der typischen intermedia und nicht mit der von DE MAN als var. amboinensis angeführten Form, die aber vielleicht nur als Jugendform aufzufassen ist.

Amboina, 2 Ex. (SEmon coll.).

Verbreitung: Mergui-Inseln und Amboina (DE MAN).

Gebia (Gebiopsis) hexucerus nox. spec. Tafel III, Fig. I.

Körner des vorderen Theiles des Cephalothorax gegen die Stirn grösser, dörnchenartig werdend. Stirn zwischen den Vorderenden der seitlichen Furchen vorspringend, daselbst mit sechs Dörnchen besetzt, von denen die beiden mittleren dicht neben einander stehen, während je die beiden seitlichen von diesen etwas ferner stehen und ebenso weit unter sich und auch von den Dornen, die die seitlichen Furchen nach aussen begrenzen, entfernt sind. Alle diese Dörnchen sind kurzbogenförmig aufgerichtet.

Nerus der Scheerenfüsse am Unterrande mit ähnlichen feinen Dörnchen wie $G$. intermedia, aber nur im proximalen Theil. Carpus unbewehrt, d. h. es fehlen die kräftigeren Dornen der $G$. intermedia oben und unten. Dagegen findet sich oben ein ganz feines Stachelchen, sowie einige Höcker. Scheerenfinger ziemlich schlank, ähnlich denen von G. intermedia. Carpus der 5. Pereiopoden etwa so lang wie der Propodus.

Stiele der inneren Antennen auffällig kürzer als die der äusseren, kaum bis zum Ende des vorletzten Stielgliedes der letzteren reichend.

Telson ähnlich dem ron $G$. intermedia. aber die seitlichen Leisten sind schwächer, nebst der vorderen auf der Oberfläche nicht rauh. Die Vertiefungen sind undeutlich.

Unterscheidet sich durch die Bildung der Stirn ron sämmtlichen übrigen Arten. Das Längenverhältniss der Stiele der inneren und äusseren Antennen ist ähnlich wie bei $G$. isodactyla, mit der sie bis auf den Bau der Stirn sonst die meiste Aehnlichkeit zeigt.

Thursday I sland, 2 Ex. (SEMox coll.).

\section{Culliunass! mucronatu STRAHL.}

Strahl, Mon. Ber. Ak. Wiss.. Berlin 1861. p. 1056. A. Milne-Enwards, Nonv. Arch. Mus., II, 1870, p. 94. de Man, Arch. f. Naturg., 53. 1, 1887 , p. 484, pl. 21, fig. 2. Ortuaxx, Zool. Jahrb., VI, 1, 1891, p. 57.

In der Länge des Stirnstachels scheint diese Art zu variiren. Das eine der mir jetzt vorliegenden Exemplare stimmt völlig mit der Abbildung bei DE MLAN überein, ein anderes (das grösste) zeigt einen noch

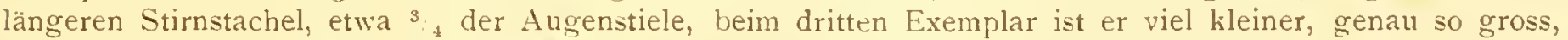
wie bei dem von mir (1. c.) von den Malediven erwähnten Exemplare.

Amboina, 3 Ex. (SEMon coll.).

Verbreitung: Malediven (Ortalann); Amboina (DE Mar); Philippinen (StrahL).

\section{Familie: Galatheilae.}

\section{Galatheu elegans ADAMS et WHITE.}

Gal. elegans, Adans et White, Zool. Top. Samarang. Crust., 1848. pl. 12, fig. 7. Haswell, Catal. Austr. Crust., 1882, p. 163. Miers, Rep. Zool. Coll. Alert, 1884, p. 278. de Max, Arch. f. Naturg., 53, 1, 1887, p. 455.

Gal. grandirostris Stimpsox, Proceed. Acad. Nat. Sc., Philadelphia 1858, p. 252.

G. longirostris DANA (U.S. Expl. Exp., I852, p. 482, pl. 30, fig. II) von den Fidji-Inseln unterscheidet sich nur durch etwas kürzeres Rostrum, das an den Seiten etwas weniger Zähne besitzt. Ich möchte sie mit dieser Art vereinigen.

Ischium des 3. Maxillarfusses etwa so lang als der Merus. Merus mit 2 Dornen am Innenrande, der eine am distalen Ende, am Aussenrande ohne Dornen. Erste Pereiopoden mit Epipodit (Mastigobranchie), die übrigen ohne solche.

Für die von mir (Zool. Jahrb., VI, I892, p. 248-49) angeführten Arten habe ich betreffs des Vorhandenseins von Epipoditen nachzutragen: C. australiensis und orientalis besitzen nur am I. Pereiopodenpaar Epipoditen, G. affinis überhaupt keine an Pereiopoden. Die Arten der Gattung Galathea bilden somit drei Gruppen: I) Mit 3 Epipoditen an den drei ersten Pereiopoden: G. squamifera, G. nexa (identisch mit letzterer ist wohl G. dispersa), 2) Mit nur einem Epipodit an dem ersten Pereiopoden: G. intermedia, australiensis, orientalis, elegans. 3) Ohne Epipoditen an Pereiopoden: G. strigosa, affinis. 
Amboina. I Ex. (SEMros coll.).

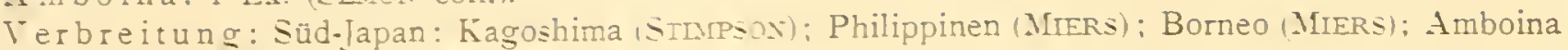
(DE MAN); Queensland (HASWELL. MIIER:.

\section{Gattung: Murida.}

Die vorliegende Art gehör in eine Gruppe der Gattung, die sich durch eine Querreihe ron Dörnchen hinter dem Stirnrande auszeichnet. Die Arten diezer Gruppe lassen sich in folgender Weise unterscheiden:

a. Abdomensegmente ohne Dornen.

aa. Abdomensegmente mii Dornen am Torderrande.

b. Augen sehr kleir. Y. japonica STPS. ').

bb. Augen grösser.

c. Zwei Dornen aü dem zweiten Abdomensegment, die übrigen Segmente ohne Dornen. (Bismeilen fehlen auch die aut dem zmeiten.) M. edicardsi MRs.s, M. inormata HEND.t).

cc. Sechs Dornen aut dem zweiten Segment, sonst keine.

d. Supraoculardornen viel bürzer als die Augen. Merus des 3. Maxillarfusses mit 2 Dornen.

M. heteracantha ORTM. ${ }^{5}$.

dd. Supraoculardornen so lang wie die Augen. Merus des 3. Maxillarfusses mit 3 Dornen. Dornen des Cephalothorax und Abdomen klein.

1. semoni nor. spec.

ddd. Supraoculardornen länger als die Augen. Merus des 3. Maxillarfusses mit 3 Dornen. Dornen des Cephalothorax und Abdomen aufiallig gross.

11. spinosa Hevi. ${ }^{\circ}$ ).

ccc. Acht bis zehn Dornen auf dem 2. Segment, sonst heine.

ע. militaris HExD. ${ }^{7}$ ) M. sanctitauli HEND. ${ }^{3}$ ).

cccc. Zweites und drittes Abdomensegment mit Dornen.

H. miles A. M.E. ${ }^{9}$, M. spinulifera MRs.10) M.

Hierher würden auch M. Epinifrons HExD." ) und M. tuberculata HExD. ${ }^{3}$ ) gehören, die ganz eigenthümliche Merkmale ron den übrigen unterscheiden.

Iunida semoni nor: spec. Tafel I, Fig. 4 .

Cephalothorax mit Rostrum I5 mm lang, das Rostrum ist $5 \mathrm{~mm}$, die Supraoculardornen $2,5 \mathrm{~mm}$ lang.

Rostrum etwa halb so lang als der übrige Cephalothorax. gerade vorgestreckt. an der Spitze schwach aufgebogen, etwa doppelt so lang als die Supraoculardornen, die schmach aufwärts gerichtet sind. Hinter dem Rostrum auf der Gastricalgegend eine Querreihe ron I3 Dörnchen, eines davon in der Mittellinie. Tederseits das zweite ron der Mittellinie an steht hinter dem Supraoculardorn und ist grösser. Dicht hinter dem Dörnchen in der Wittellinie steht ferner noch ein Dörnchen. Vorderseitenechen des Cephalo. thorax mit einem langen Dorn. auf diesen folgen am Seitenrande vor der Cervicalfurche 3 Dörnchen, ron denen das rorderste rudimentär ist. hinter der Cervicaliurche stehen noch 5 Dörnchen. Auf der Gastricalgegend steht hinter dem Dorn der Torderseitenecken noch ein Dörnchen, und ferner finden sich zwei weitere schrāg hinter einander dicht hinter der Cerricalturche. Der hintere Theil des Cephalothorax ist unberrehrt.

Zweites Abdomensegment am Torderrande mit 6 Dörnchen, die zwei medianen der Mittellinie genähert. je die zwei seitlichen einander genähert und ron den medianen entfernt. Die übrigen Abdomensegmente ohne Dörnchen.

Augen mittelmāssig. die Supraoculardornen nicht überragend, mit kurzen Haarfransen. Aeussere

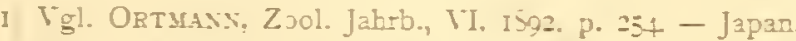

- Vigl. Hexdersos, Chall. Anomur., ISjs, p. I 2\%. - Atlantic Antarcic.

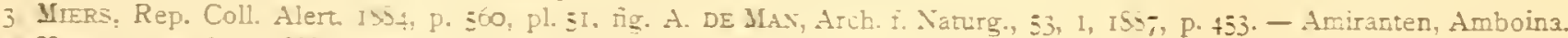

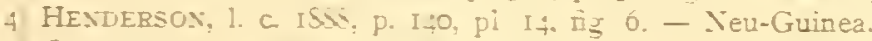

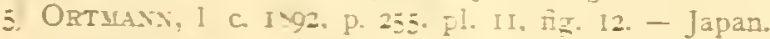

6 Hevdersox. l. c IS5S. p. IzS. pl. 3, fig. 3. - Sud-Atlantic, Antarctic.

7 HeNDDRSON, !. c. IAS:. p. I37. pl. If, fig. 2 und 5. - In Jischer Archipel, Pacific

S HeIdERSOX. 1. c. Iss:, p. I 42 . jl. 3. fig 6. - St. Paul's Rock.

9 Vgl. HeNDERSON, 1. c. I355. p. I 25. - West-Indien.

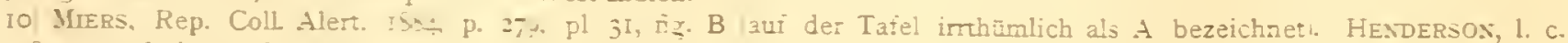
Is55, P. I2S. - Amboina und Aratura-See.

II HeNDERSON, 1. c. ISS3, p. I 43 . pl. I 4. fig. 4. - İeu-Seeland

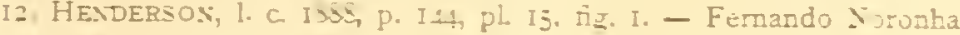

I3 HeNderso:, L c. IS39. p. I45. p.. I5, nis. 2. - Pacific 
Antennen mit zwei schlanken Stacheln. Ischium des dritten Maxillarfusses am distalen Ende des Unterrandes mit einem Stachel, Merus am Unterrand mit drei Stacheln.

Scheerenfüsse des ¿ schlank. fast cylindrisch, mit schuppigen, nur wenig bedornten Gliedern. Palma länger als der Carpus. Finger etwa so lang als die Palma, zusammenschliessend. Zweite, dritte und vierte Pereiopoden mit am Ober- und Unterrand dornigen Meren und Carpen, Propoden nur am Lnterrand dornig.

Sexualanhänge beim ¿ vorhanden.

Am boina, 2 Ex. (SEMor coll.).

\section{Familie: Poreellanidae.}

\section{Gattung: Petrolisthes.}

Die ron mir (Zool. Jahrb., V1, IS92, p. 258) zusammengestellte Gruppe A.. B. dieser Gattung, also die Arten, wo der Vordertheil des Cephalothorax nur vor den Augen etwas abwärts geneigt ist, wo der Carpus der Scheerenfüsse am Vorderrand einen oder mehrere Zähne besitzt. und no der Cephalothorax unbehaart ist und auch keine scharfen Querlinien zeigt, würde ich nunmehr in folgender WTeise gliedern:

A. B.

C. Hinterrand des Carpus der Scheerenfüsse mit ungezähnter Leiste, die an der distalen Ecke als Dorn vorspringt. Ohne Epibranchialstacheln.

D. Oberfläche des Cephalothorax höckerig. Torderrand des Carpus der Scheerenfüsse nur am proximalen Ende mit einem Zahn ${ }^{1}$ ). $\quad$ P. mossambicus (HLGDF.12). P. indicus DE MAx ${ }^{3}$ ).

DD. Oberfläche des Cephalothorax nicht höckerig. Vorderrand des Carpus mit mehreren Zähnen. E. Meren der hinteren Pereiopoden oben und unten ohne Dörnchen. P. leptocheles (HELL. $)^{4}$ ).

EE. Meren der hinteren Pereiopoden oben und unten mit Dörnchen in variabler Anzahl.

F. Scheeren gedrungener. Zähne des Carpus schärfer.

F.F. Scheeren schlanker. Zähne des Carpus schwach und wenig rorragend.

P. tentiatei D. M( $\left.{ }^{6}\right)$.

CC. Hinterrand des Carpus der Scheerenfüsse mit einer in mehr oder weniger grossen Ausdehnung gezähnten Leiste.

D. Vorderrand des Carpus nur am proximalen Ende mit einem Zahn, selten noch entfernt ron dem ersten in der Mitte mit einem zweiten, spitzen Zahn. Hinterrand am distalen Ende mit einer Reihe ron zwei bis drei Zähnen. Ohne Epibranchialstachel. P. japonicus (D. H.) ${ }^{\top}$ ).

DD. Vorderrand des Carpus mit mehreren, genäherten Zähnen. Auch am Hinterrand meist mehr Zähne.

E. Meren der hinteren Pereiopoden am Oberrand ohne Dörnchen. Epibranchialstachel rorhanden oder fehlend.

EE. Meren der hinteren Pereiopoden am Oberrand mit Dörnchen. Epibranchialstachel vor. handen.

I) Hierher rielleicht: P. untolatus Hexdersox, Chall. Anom., 19ys. p. Io6, pl. II, fig. 3. ron Tongatabu, mit nur einem Zahn am Vorderrand des Carpus und nicht höckerigem Cephalothoras.

2 Porcellara mossambica Hilgexdorf. Mon. Ber. Ak. Wiss. Berlin, I5-5, p. Sz5, pl. z, fig. 6. - Mozambique.

3) DE Max, Not. Leyd. IIus., 15. IS93, p. 293. pl. 7 , fig. 3 (Vgl. daseibst die Únterschiede von roriger Art.) - Flores.

4) Porcellana leptocheles Heller, Sitz. Ber. Ak. Wiss. Wien, H1, IS6I, p. 25S. pl, 2, fig 6. Vgl auch DE MfAx, Xot. Lexd. Mus., 15. 1S93, P. 292. - Rothes IIeer.

5) Vgl. ORTMAxx, 1. c. IS92. p. 260, nebst den dort angregebenen Symonymen. Die Exemplare des hastatus, die mir ron den Liu-Kiu-Ins. vorliegen, variiren in der Bedornung der Jeren der hinteren Pereiopoden. Ich finde z. B. auf den 2. und 3. Pereiopoden distal ein Dömchen, dann auf den 2.. 3. und +. Pereiopoden ein ebensolches Dörnchen, und ferner (bei grossen Exemplaren) auf den 2. und 4 oben ein distales, auf dem 3 oben zwei. ein distales und ein proximales. Ich muss also hastatus und inermis für identisch halten. - Liu-Kiu-Ins., Jara, Flores, Mergui-Ins.

DE MAN (Journ. Linn. Soc., 22, ISS5, p. 2I 4 ) hat ron A. MILxE-EDWARDs ein angeblich typisches Exemplar der Porc. lamareki

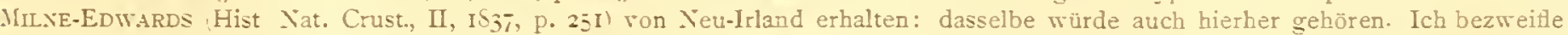
aber, dass das Exemplar typisch war, denn es entspricht nicht der Beschreibung in der H. X. Cr., p. 23I. Zeile 23 r. 0. ., wo wörtlich steht: „L"ne petite crête denticulée au dessus de son bond postérieur" (i. e. du carpe). Nach diesem Satze kann lamarcki nicht in die hastatus-Gruppe gehören V'gl. unten.

6) DE MAx, Not. Leyd. Ilus.. 15. 1593. p. 2S9, pl. 7, nig. 2. - Flores.

7) Vgl. ORtMaxi, l. c. p. 261 (mit Synongm: inermis Hasmeli. - Japan. Liu-Kiu-Ins., Bonin-Ins. China, Australien, Mergui-Ins. - In Neu-Seeland wird diese Art ron Petr elongatus . I.-E.) ersetzt, der sich durch gedrungenere Carpen der Scheerenfüsse und weniger schlanke Gehfüsse unterscheidet. Auch ist bei letzterem der Seitenrand des Cephalothorax schärfer und die Gastricalgegend trăgt zwei schwache Erhabenheiten. Vgl. Hssirel, Catal. Austral. Crust.. ISSz. p. I40. 
F. Zweite bis vierte Pereiopoden oben mit je einem Dörnchen nahe dem distalen Ende. Carpus der Scheerenfüsse schlanker, zweimal so lang wie breit. P. leporinoides OrtM.').

FF. Zweite bis vierte Pereiopoden mit je einer Reihe von 5-6 Dörnchen. Carpus der Scheerenfüsse gedrungener, I $\frac{1}{2}$ mal so lang wie breit.

P. moluccensis D. M. ${ }^{2}$ ).

\section{Petrolisthes lamareki (LEACH).}

Pisidia lamarckii LeAcr, Diction. Scienc. Natur., t. 18, 1820, p. 54.

Pisidia asiatica Leach ibid.

Porcellana lamarckii (Leach) Milne-Edwards, Hist. Natur. Crust., II, 1837, p. 251.

Porcellana dentata MiLne-EDwards, ibid.

Porcellana speciosa DANA, U. S. Expl. Exp. Crust., 1852, p. 417, pl. 26, fig. 8.

Petrolisties speciosus (DAn.) Stimpsos, Proceed. Acad. Nat. Sc. Philadelphia, 1858, p. 241.

Porcellana rufescens Heller, Sitz. Ber. Akad. Wiss. Wien, 44, 1861, p. 255, pl. 2, fig. 4. Heller, Crust. Novara, 1865 , p. 76.

Porcellana bellis Hellen, Novara, 1865, p. 76, pl. 6 , fig. 4.

Porcellana (Petrolisthes) rufescens Hell. Hilgexdorf, Mon. Ber. Ak. Wiss. Berlin, 1878, p. 825, pl. 2, fig. 7.

Petrolisthes dentatus (M.-E.) HAswell, Catal. Austral. Crust., 1882, p. 146.

Petrolisthes lamarchi (Leach), Mrens, Rep. Zool. Coll. Alert, 1884, p. 268 und 557.

Petrolisthes lamarchi var. asiaticus (LEACH) Mienrs, ibid. p. 269 und 557.

Petrolisthes haswelli Mrens, ibid., p. 296, pl. 29, fig. A.

Porcellana (Pethrolisthes) dentata DE MAx, Arch. f. Naturg., 53, 1, 1887, p. 409, pl. 18, fig. 7.

Porcellana dentata de Max, Journ. Linn. Soc. Zool., 22, 1888, p. 216.

Petrolisthes dentatus (M.-E.) Ontmaxi, Zool. Jahrb., VI, 1892, p. 262.

Petrolisthes speciosus (Dan) ORTMans, ibid.

Ich habe (I. c.) den I'. speciosus von deutatus getrennt, indem ich wesentlich auf das Vorhandensein oder Fehlen des Epibranchialstachels Rücksicht nahm. Neuerdings hat mir DE MAN (Not. Leyd. Mus., 15, 1893, p. 296, Anmerk.) vorgeworfen, dass ich auf seine Angaben keine Rücksicht genommen habe, und die Form o h ne Epibranchialstachel mit dem Namen dentatus M.-F. belegt habe, während ihm von A. MiLnEEDWARDS mitgetheilt sei (cf. DE MAN, I888, p. 216), dass die Exemplare von den Mergui-Inseln, die einen Epibranchialstachel besitzen, mit den Originalen der $I$. dentata M.-E. übereinstimmen.

Abgesehen davon, dass ich dieser Angabe von A. MILNE-EDWARDs absolut keinen Werth beimesse. ${ }^{3}$ ), musste ich, falls die dentata wirklich einen solchen Stachel besitzt, nach den Regeln über zoologische Nomenclatur die $I$. dentata der Hist. Nat. Crust. als ungenügend charakterisirt, als nomen nudum behandeln, da ja 1I. Milne-EDTARDS 1. c. einen Epibranchialstachel bei anderen Formen erwähnt (z. B. I. rugosa p. 252, maculata p. 253). Für die mit einem Epibranchialstachel versehene Form musste ich denjenigen Namen wählen, unter dem sie zuerst kenntlich beschrieben wurde, und das ist: Porcellana speciosa DANA $1852^{4}$ ). Dass späterhin, I888, von DE MAN nachgewiesen wurde, dass die Originale der dentuta M.-E. ebenfalls diesen Stachel besitzen, kann an der ganzen Sache nichts ändern, da etwa noch vorhandene Originale nicht maass. gebend sein können, wenn die ursprüngliche Beschreibung mangelhaft ist, und inzwischen bessere publicirt worden sind.

Für die Form mit Epibranchialstachel ist also unter allen Umständen der Name: Petrolisthes speciosus (DANA) zu wählen.

Eine zweite Frage ist, ob ich für die Forn ohne Epibranchialstachel den zum nomen nudum gewordenen Namen: dentata wieder anwenden durfte; im Allgemeinen kann man ein derartiges Verfahren nicht befürworten. Im vorliegenden Falle ging ich aber von anderen Erwägungen aus. Der ältere MILNEEDWARDS führt bei mehreren Arten der Gattung Porcellana Epibranchialstacheln auf, bei dentata erwähnt er nichts davon: ich musste also annehmen, dass solche hier fehlen, besonders da mir thatsächliche derartige Exemplare vorlagen, und ich war dann völlig im Recht, für diese Exemplare den Namen dentata anzuwenden. Selbstverständlich musste ich dann die Richtigkeit der von A. MilnE-EDwARds an DE MAN gemachten Mittheilung bezweifeln.

Es lagen also zwei Möglichkeiten vor: en $\mathrm{tweder}$ hatte A. MilNE-EDwards Recht, dann ist dentata ein nomen nudum, die stacheltragende Form musste den Namen: speciosus erhalten, und für die stachellose musste ein anderer gefunden werden. Das wäre in diesem Falle $P$. rufescens HeLLER IS6I gewesen: die Identität dieser Form mit der vorliegenden war mir damals entgangen.

1) ORTMANN, 1. c. p. 263 . - Südsee.

2) DE MAN, Arch. f. Naturg., 53, 1, 1887, 13. 411 , pl. 18, fig. 5. - Amboina.

3) Für die Unzuverlässigkeit A. MrLnE-EDWARDS' betreffend derartiger Angaben und auch im Allgemeinen habe ich nunmehr soviel Beispiele gesammelt, dass ich persönlich sämmtlichen Angaben desselben ein gewisses Misstrauen entgegenbringe.

4) Diese Beschreibung ist vollkommen genügend, wenn auch die dazu gehörige Abbildung Mängel besitzt (pl. 26, fig. 8j. 
Oder die Angabe A. Milne-Edwards' ist unrichtig: dann musste ich so verfahren, wie ich 1 . c. gethan habe.

Diese ausführliche Auseinandersetzung halte ich deshalb für nothwendig, um meine damalirge Benennung der verschiedenen Formen zu rechtfertigen, und um nachzuweisen, dass ich schon damals mit voller Kenntniss der Angaben DE MAN's vom Jahre I888 für die mir vorliegenden Formen die betreffenden Namen wählte, was ja schon theils aus den Citaten, theils aus dem unter Petr. dentatus Gesagten (l. c. p. 262) hervorgeht.

Demgegenüber bin ich aber nunmehr zu der Ueberzeugung gekommen, dass die Form mit und die ohne Epibranchialstachel völlig identisch sind, dass das Fehlen dieses Stachels nur zufällig ist, eventuell als Variation aufzufassen ist, und zwar aus dem Grunde, weil die mir vorliegenden Exemplare ohne Epibranchialstachel in allen übrigen Einzelheiten sowie im ganzen Habitus so vollkommen mit den bestachelten übereinstimmen, dass es mir völlig unmöglich ist, irgend welchen weiteren Unterschied aufzufinden. Ich gehe jetzt sogar noch weiter und halte die Porc. lamarcki für identisch mit diesen Formen, da sie sich einzig und allein durch nur drei Zähne an Vorderrand des Carpus der Scheerenfüsse von den übrigen Formen unterscheidet, vgl. Milne-EDwARDs, I837, p. 25 I und Miers, Alert, I884, p. 268. Diesen Beschreibungen gemäss ist P. lamarcki auf jugendlichere Exemplare gegründet, und gerade bei jüngeren Exemplaren kommt nach DE MAN ( I888, p. 2I6) diese geringere Zahnzahl vor.

Wenn das Fehlen oder das Vorhandensein des Epibranchialstachels in diesem Falle unwichtig ist, alsdann sind die Diagnosen des älteren MiLne-Fowards genügend, um die Art zu erkennen, und es muss als ältester Name: P. lamarcki angewendet werden. Zwar ist die Beschreibung bei LEACH, I820, völlig unzureichend, jedoch nimmt MisNe-FDwards 1837 diesen Namen auf, ohne dass in der Zwischenzeit die in Frage kommenden Formen anderweitig besprochen wurden. I'. asiaticus LEAcH ist nach den Angaben bei Miers ebenfalls hierher zu ziehen.

Von Ost-Afrika liegen mir drei mit Epibranchialstachel versehene Exemplare vor, die ich unter einem und demselben Korallblock sammelte: das jüngste von ihnen würde der P. lamarcli, mit nur drei Zähnen am Carpus, entsprechen.

Dar-es-Sal a am, Upanga-Riff, an Korallen, 37 (ORTMAN coll.).

Verbreitung: Ueberall im Indo-pacifischen Gebiet.

Rothes Meer (Heller); Mozambique (Hilgendorf); Amiranten: Darros Isl. (Miers); Mauritius (Leach); Persischer Meerbusen (Heller); Ceylon (Miers); Nicobaren (Heller); Mergui-Ins. (DE Man); Keeling Isl. (Mliers); Java (M.-E., D. M.); Balabac-Strasse (DANA); Philippinen (MiERs); Insel 'Ty-pin-san (Miers); Hongkong (Stmipson); Liu-Kiu-Ins. (Stmpson); Süd-Japan: Kagoshima (Stmpson); Bonin-Ins. (Stinipsov); Thursday Isl. (Mıers); Ost-Australien (Ortr.): Queensland (Haswell, Miers); Kingsmill-Gruppe (Dand); Tahiti (Heller); Paumotu-Arch. (Dana); Wake-lns. (Dasi).

Die folgende Art Petr. trivirgatus) bildet mit mehreren anderen Arten der Gattung eine Gruppe, die sich durch behaarten Cephalothorax, sowie durch eine Dörnchenreihe am Aussenrande der Palma auszeichnet. Diese Gruppe entspricht der von nir (1. c. 1892, p. 259) mit BB. CC. bezeichneten Abtheilung, die 1. c. die Arten: villosus, tomentosus und militaris enthält. Alle diese drei haben jene Dörnchenreihe am Aussenrand der Palma, und es kommen ferner noch einige hinzu, die sich folgendermaassen zusammenstellen lassen:

CC. Cephalothorax ohne scharfe Querlinien, behaart. Aussenrand der Palma mit einer Dörnchenreihe.

D. Ohne Supraocularstachel und ohne Epibranchialstachel. Hinterrand des Carpus der Scheerenfüsse ohne Dörnchen.

E. Cephalothorax dicht-filzig, mit Büscheln längerer Haare. Meren der Pereiopoden (besonders der dritten) im Alter oben mit ein bis zwei Dörnchen. P. villosus Richt. ${ }^{1}$ ). EE. Cephalothorax kurzhaarig, nicht filzig. Merus der 2.-4. Pereiopoden oben ohne Dörnchen.

$P$. trivirgatus $n$. $s p$.

DD. Olne Supraocularstachel, aber mit Epibranchialstachel. Cephalothorax behaart. Carpus der Scheerenfüsse nahe dem Hinterrand mit 2-3 Dörnchen, die auf die Oberfläche gerückt sind (wie bei barbatus), distales Dörnchen zweispitzig. Merus der 2.-4. Pereiopoden oben mit mehreren Dörnchen, unten distal mit $\mathrm{I}-2$ Dörnchen.

P. tomentosus (DAN. $\left.)^{2}\right)$.

DDD. Mit Supraocular- und mit Epibranchialstachel.

i) Vgl. Ortmann, 1. c. p. 264. Miers, Alert, iSS4, p. 559. - Mauritius, Amiranten, Fidji.

2. Vgl. Ortmaxi, ibid. p. 264. - Paumotu-Ins. 
E. Keine weiteren Stacheln auf dem Cephalothorax.

F. Eine Reihe ron 2-3 Dörnchen nahe dem Hinterrand des Carpus auf dessen Oberfläche, das distale 2 reispitzig. Carpus der 2. Pereiopoden oben distal mit einem Dorn.

FE Fine Reihe ron 4 Dörnchen am Hinterrand des Carpus selbst. das distale zweispitzig. Carpus der 2. Pereiopoden oben ohne Dorn.

P. carinipes (HELL.) :).

EE. Cephalothorax noch mit meiteren stacheln.

F. Seitenränder des Cephalothorax ausser dem Epibranchialstachel noch mit 3 Stacheln. Seitliche Stirnlappen ohne Stacheln.

FF. Seitenränder des Cephalothorax mit mehr (5-6) Stacheln. Seitliche Stirnlappen mit mehreren Siachelchen besetzt.

P. militaris (HELL.) ${ }^{\mathrm{s}}$ ).

Petrolisthes trivirgatus nox. spec. Tafel II, Fig. 5.

Cephalothorax länger als breit. Stirn dreilappig, vorragend. Oberseite des Cephalothorax flach, kurzhaarig. besonders die Stirn dicht behaart. Epibranchialstachel und Supraocularstachel fehlend.

Scheereniüse ebenfalls kurzhaarig. Aussenrand der Hand lang behaart und mit einer Reihe ron 5-6 Dörnchen besetzr. Vorderrand des Carpus mit drei spitzen Zähnen, Hinterrand ohne Zähne. Hintere Beine ebenfalls kurzhaarig, ihre Neren am Oberrand ohne Dörnchen.

Färbung charabteristisch: unten heller. oben dunkel-riolett. Cephalothorax oben mit drei meissen Längsstreifen, die sich auf der Stirn rereinigen. Scheerenfüsse und hintere Füsse mit weissen und violetten Längsstreifen. Abdomen mit meissen Querstreifen. Behaarung gelblich.

Dar-es-Sala am. Lpanga-Riff, an Korallen. I 2 - ORTMAxi coll.

Pisisoma gramulatum nor. spec. Tafel 1I. Fig. 6.

Cephalothorax gewölbt. fasi kreisrund. Oberfläche fein granulirt. Stirnrand von oben besehen abgerundet, ron rom besehen schwach dreilappig. Torderseitenrand als feine Linie erkennbar.

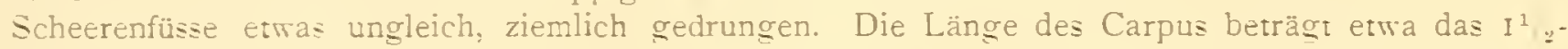
bis 2-fache der Breite: Carpus ziemlich dick, sein Hinterrand gerundet mit einer undeutlichen Leiste. Jorderrand abgeflacht, mii + bis 5 tlachen, dreieckigen Zähnen, die gegen das distale Ende an Grösse abnehmen. Hand dreiechig. etwas geschwollen. Oberseite ron Carpus und Hand fein granulirt.

Hintere Beine gedrungen, granulirt. sehr schwach behaart. Uebriger Körper unbehaart.

Farbe im Leben dunkelgrün, in Alkohol hell mit dunklerer Marmorirung.

Bildet den Lebergang von Petrolisthes zu Pisisoma. da die Stirn noch eine Spur ron Drei-Theilung zeigt. Auch die äusseren Antennen sind ganz wie bei typischen P'etrolisthes gebildet. Durch die gedrungenen. dicken scheeren und den rundlichen, zewölbten Cephalothorax steht die Form jedoch Pisisoma näher. Vigl. jedoch auch daz unter Pachycheles Gesagte.

Dar-es-Salaam, Ras Rongoni, $2 Z+=$, Chokirbank, I + , in Korallkalk (OrTMAx coll.).

\section{Gattung: Iruchycheles.}

Stupsox (Proceed. Acad. Nat. Sc. Philadelphia, I559, p. 227-229) unterscheidet innerhalb der Familie der Porcellaniden zwei Gruppen: A. Erstes Glied der Antennen kurz, den oberen Rand des Cephalothorax nicht erreichend. B. Erstes Glied der äusseren Antennen mehr weniger vorgezogen und mit dem Rand des Cephalothorax vereinigt, zweites Glied ron der Orbita entfernt. Zu A. gehört Petrolisthes, der das Terhältniss typisch zeigt, somie Pisisoma, das sich nur durch gerade Stirn, mehr gerundeten Cephalothoras und dicke Scheerenfüsse unterscheiden soll. Die übrigen Gattungen: Paphidopus, Pachycheles. Megalobractium, Porcellana, Minyocerus, Porcellanella, Polyonyx gehören zu B.

Zunächst kann ich für Fixplidopus, Porcellana und Polyonyx das ron STIMPsox angegebene Terhalten bestätigen. Bei diesen ist das erste Glied der äusseren Antennen gut entwickelt und quer verbreitert, tritt deutlich zwischen dem Auge und der Vorderecke der Epimeren hervor, und stösst nach oben an die äussere

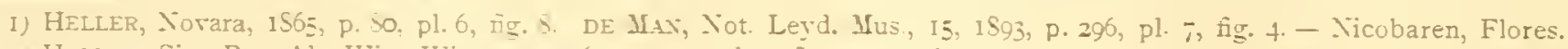

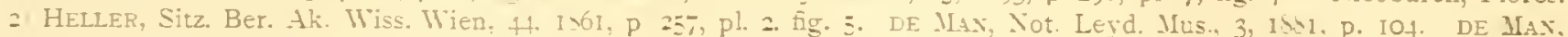
ibid. 15,1 , 93 , p. 299, pl. 7, rig. 5. - Rothes Meer

3 Vgl. ORTMAM: 1. c. p. 265 - Nicobaren, Java. Liu-Kiu.

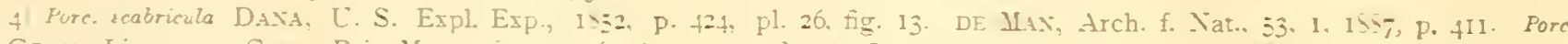

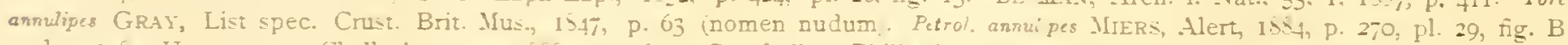
und p. इミs. Hexdersox, Chall. Anomur., 1855, p. 106. - Seychellen, Philifpinen. Sulu-See, Amboina, Australien. 
Ecke des oberen Orbitalrandes und bleibt ron da noch eine gewisse Strecke mit dem Seitenrand in Berührung. Dadurch wird das 2. Stielglied der Antennen von den Augen völlig getrennt.

Bei der Gattung P'achycheles soll nach Stmipsox das erste Stielglied der äusseren Antennen weniger rorgezogen sein. Bei den mir rorliegenden, auch von Stimpsox als typisch angeführten Arten: $I$. natalensis und stevensi kann ich ebendies beobachten, und zwar so, dass einerseits das erste Stielglied zwar als kurzer querer Kiel besser sichtbar ist als bei l'etrolisthes, andererseits es sich aber nicht mit der äusseren Ecke des oberen Orbitalrandes vereinigt, so dass zwischen ihm und dieser Ecke noch eine Lücke bleibt. in die sich ein kleiner Fortsatz des zweiten Stielgliedes einfügt: dieses Glied ist also nicht völlig ron den Augen getrennt. Demnach würde Pachycheles besser in die Abtheilung A. zu stellen sein: jedenfalls bildet sie in der Bildung der Antennen einen Uebergang von Petrolisthes zu Porcellan und Verwandten.

Ein ganz auffallendes. Merkmal, das alle mir vorliegenden, zu Pachycheles gerechneten Formen zeigen. ist folgendes: ron den Epimeren des Cephalothorax ist hinten ein besonderes, drei- oder schief-vierechige: Stück durch eine häutige Naht abgetrennt. Dieses Merkmal halte ich für äusserst charakteristisch, da ebei keiner der übrigen Gattungen, die mir bekannt sind, vorkommt, und gerade wegen dieses Merkmals rechne ich die Porcellana sculpia MI.-E., die von Stmpsox zu Pisisoma gestellt wurde, ebenfalls zu Pachycheles. Ein mir porliegendes Exemplar der $P$. sculpta von den Liu-Kiu-Inseln (ORTMAx, 1. c. p. 265) zeigt ebenso wie das mir jetzt vorliegende, sicher mit P'ach. natalensis identische. einerseits dieses losgelöste Epimerenstück, andererseits stimmt es in der Bildung der Antennen mit den anderen Pachycheles-Arten überein.

Die mir vorliegenden Arten: lnevidactylus, sculpius und pulchellus zeigen an der Unterseite der Krallen hornfarbige Nebendornen, bei stevensi sind diese zu bleinen Höckern reducirt. Nach Strupsoy sollen die Krallen bei Pachycheles normal sein.

Die Arten der Gattung Pachycheles lassen sich in folgender Weise zusammenstellen:

a. Scheeren und Carpus granulirt. Die Granulationen bilden keine Reihen oder Längswülste aut der Hand.

b. Scheeren oben behaart.

F. barbutus A. M..E. ${ }^{1}$.

bb. Scheeren oben unbehaart.

c. Granulationen der Hand fein. Beweglicher Finger glatt. Carpus am Vorderrand zweizähnig.

cc. Granulationen gröber, auch auf dem beweglichen Finger vorhanden. Carpus vorn dreizähnig. 1'. stevensi STPS. 1 .

aa. Scheeren und Carpus granulirt, die Körner Längs-Reihen oder - Wülste bildend.

b. Granulation grob, auf Carpus und Scheere einfache Reihen bildend.

bb. Granulation feiner, auf der Scheere zu 2- 4 Wülsten zusammentretend. aaa. Scheeren nicht granulirt, mit + glatten Längswülsten.

P. moniliferus (D+x, $)^{\div}$, H. sculptue (M.-E.) P. pulchellus 1 HAsw.

\section{Puchycheles smulptus (MIILNE-EDWards).}

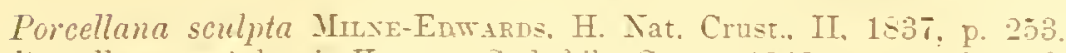

Porcellana natalensis KRscs, Sitdatrik. Crust., 1st3. p. 5o, pl. t. fig. 1

Pore. (P'isis.) sculpta M.-E. DE Max, Arch. f. Taturg. 53. 1. 185i. p. 413.

Pisisoma sculptum (M.-E.) Ortmaxx. Zoolog. Jahrb., VI, 1892. p. 2055.

Ob Porc. sculpta bei DAxA (L. S. Expl. Exp., I852, p. 412. pl. 20, fig. 2) ron Fidji, Sulu.See. Balabac-Str. hierher gehört, ist nicht ganz sicher, da in der Abbildung die Körner der Scheere zu gross erscheinen. Vielleicht ist dies aber nur einer Lngenatigkeit der Abbildung zuzuschreiben.

DE MAx will hiermit den $P$. pulchellus vereinigen. Ihm lagen von Jara Exemplare ror, die diesen Art entsprechen, von den Mergui-Inseln dagegen solche, die dem $H^{\prime}$. pulchellus entsprechen. Ich halte beide für verschieden, wenn sie sich auch sehr nahe stehen. Porc. natalensis ist unzweifelhaft hiermit identisch. das vorliegende Exemplar stimmt vollkommen mit Beschreibung und Abbildung bei KRt $\mathbf{L} s$, wie auch mit den übrigen Beschreibungen. Seine Färbung war im Leben gleichmässig röthlich-braun.

Dar-es-Sala am, Cpanga-Riff, an Korallen, I (ORTMAris coll.).

Verbreitung: Natalküste (Kratss); Java (Milse.Edwards): Ins. Soordrachter und Edam (DE MAX); Liu-Kiu-Inseln (ORTuAxx).

1) Vgl. Hexdersox, Chall. Anomur., 1858, p. IIf, pl. 11, fig. 4. - Cap Verden.

2) Ortalax, Zool. Jahrb., II, 1\$92, p. 266. pl. I2. fig. I. - Brasilien.

3) Vgl Ortmaxis ibid. p 267. - Japan.

4) DAxt, L. S. Expl. Exp.. I552. p. 413. pl. 26, nig. 3. - Brasilien. 
P'achycheles pulchellus (HaswerL).

Porcellana melchella Hasweit, Catal. Austral. Crust., 1882, p. 148.

Pachycheles pulchellus (Hasw.) Miners, Rep. Zool. Coll. Alert, 1884, p. 273, pl. 30, fig. A. Hexdenson, Chall. Anomur., 1888, p. 114.

Porcellana sculpta de Max, Journ. Linu. Soc. Zool., XXII, 1888, p. 218.

Thurs day Island, 2 Ex. (SEmon coll.).

Verbreitung: Queensland (Haswell, Henderson); Torres-Strasse (Miers); Arafura-See (HenDERSON); Mergui-Inseln (DE MAN).

\section{Polyonyx biumgmiculatus (DANA).}

Porcellana obesula Grax, List of specim. Crust. Brit. MLus., 1847, p. 130 (nomen nuduu).

Porcellana biunquiculata DAxA, U. S. Expl. Exp. Crust., I, 1852, p. 411, pl. 26, fig. 1. Haswell, Catal. Austral. Crust., 1882 , p. 147.

Polyonyx obesulus Mrens, Rep. Zool. Coll. Alert, 1884, p. 272, pl. 29, fig. D.

Porcellana (Polyonyx) obesula WH. DE MAN, Arch. f. Naturg., 53, 1, 1887, p. 423.

Polyonyx obesulus Mrs. Hexdersox, Chall. Anomur., 1888, p. 115.

Da der Species-Name obesula nomen nudum ist, muss für diese Art der DaNA'sche Namen: biunguiculata angewendet werden. Die biunguiculata bei Miers (1. c. p. 559) und DE MAN (1. c. p. 42I) vom GoIf von Suez, den Seychellen, Amiranten und Amboina ist, wie DE MAN schon ganz richtig bemerkt, nicht die biunguiculata DANA, sondern eine ne ue Art, die neu benannt werden muss.

Thursday Island, 2 \& (SEMON coll.).

Verbreitung: Queensland: Port Denison (Miers), Holborn-Ins. (Haswell), Flinders-Passage (Henderson); Torres-Strasse: Prince of Wales Channel (Miers); Nord-Australien : Port Darwin (Miers); Amboina (DE MAN); Chinesisches Meer: Madgica-Sima-Gruppe (GRAY).

\section{Familie: Paguridae.}

Prem?us imbrichtus MILNE-EDWARDS.

Minve-Edwaris, Annal. Sc. Natur. (3), Zool. X, 1848, p. 61. Miers, Rep. Zool. Coll. Alert, 1884, p. 264. Henderson, Chall. Anomur., 1888, p. 57.

Diese Art steht dem Pag. venosus M.-E. von West-Indien am nächsten. Pag. venosus hat jedoch in der Mitte der Aussenfläche des Propodus und Dactylus des 3. linken Pereiopoden eine deutliche gekörnte Längskante, die dem Pag. imbricatus fehlt. Die Schuppenskulptur dieser beiden Glieder ist bei beiden Arten ähnlich.

Thursday Island, I $d$ I $q$ (SEmon coll.).

Verbreitung: Australien: Ruffles-Bay (Milne-Edwards), Flinders-Passage (Henderson), TorresStrasse (Miers), Shark-Bay (Miers); Neu-Seeland (Miers).

\section{Pagmens munctulatus OLIVIER.}

Tgl. Ortmans, Zool. Jahrb., TI, 1892, p. 286.

A mboin a, 7 Ex. (SEMon coll.).

Dar-es-Sal a m m, Upanga-Riff, 3 Ex., Chokirbank, 2 Ex., Ras Rongoni, I Ex. (Ortmann coll.).

Lebt auf den Riffen bei Dar-es-Salaam überall zwischen Seegras und Tang.

Verbreitung: Tom Rothen Meer, Mozambique und Madagascar bis zu den Liu-Kiu-Inseln, Australien und Tahiti.

\section{Pagmens elopsis DanA.}

Tgl. Ortmani, 1. c. p. 286.

Amboina, I Ex. (SEmon coll.).

Verbreitung: Seychellen (Richters); Ins. Edam b. Java (DE MAN); Amboina (De MAN); Borneo: Balabac-Strasse (Dana); Liu-Kiu-Ins. (Ortuann); Tongatabu (Henderson); Samoa-Ins. (Dana).

Prourus setifer Mune-Edwards.

Tgl. Ortmani, 1. c. p. 287.

Dar-es-S a la am, Upanga-Riff, i $\delta$ (Ortmann coll.).

Verbreitung: Ost-Afrika, Mauritius, Ceylon, Java, Australien. 
Igl. Ortmaxs, l. c. p. 288.

Pagurus deformis Milne-Enwards.

A mboina, 3 Ex. (Semon coll.).

Dar-es-Salaam, Chokirbank und Ras Rongoni, 7 Ex. (OrTwann coll.).

Verbreitung: Von Ost-Afrika bis Tahiti.

\section{Pogurus asper" DE HAAN.}

Pagurus asper De HaAx, Faun. japon. Crust. dec. septim., 1849, p. 208, pl. 49, fig. 4. Daxa, U. S. Expl. Ex. Crust., 1, 1852, p. 450. DE MAX, Not. Leyd. Nus., 3, 1881, p. 130.

P. pedunculatus Miers, Ann. Mag. Nat. Hist. (5), T, 1880, p. 374. Haswetr, Catal. Austral. Crust., 1882, p. 155.

Diese Art unterscheidet sich von $P$. deformis und pedunculatus sofort dadurch, dass der Propodus des 3. linken Beines nur 2 Kanten hat, nämlich eine stumpfere am Oberrande und eine schärfere am Unterrande; die Aussenfläche zwischen diesen beiden Kanten ist gewölbt und glatt. Von P. deformis unterscheidet sie sich weiter durch den beweglichen Finger der grossen Scheere, der oben keinen gezähnten Kiel, sondern mehrere Körnerreihen trägt, sowie durch den Dactylus des 3. linken Beines, der auf der Aussenfläche einen Längskiel besitzt. Die beiden letzten Merkmale stimmen mit 1 . pedunculatus = varipes überein.

Miers giebt für seinen pedunculatus ausdrïcklich das Fehlen der Kiele auf dem Propodus des 3. linken Beines an: "the penultimate joint of the third left leg is smooth, not carinated." Seine Exemplare können also nur hierher gehören, da sowohl deformis als auch varipies zwischen dem oberen und dem unteren Kiel noch zwei weitere Kiele zeigen: einen scharfen nalıe dem Oberrande, der mit dem Kiel des Oberrandes eine „obere Fläche" (Hellek) einschliesst, und einen stumpfen, wulstartigen Kiel in der Mitte der Aussenfläche. Eine "obere Fläche" fehlt bei $P$. asper vollkommen.

Die drei fraglichen Arten sind also tabellarisch so zu unterscheiden. (Vgl. Ortmann, 1. c. p. 183, AAAA. B.).

C. Beweglicher Finger der grossen Scheere mit einem scharfen, gezähnten Kiel. Dactylus des 3. linken Beines auf der Aussenfläche ohne Längsleiste. Propodus mit 2 scharfen Kanten am Oberrande.

P. deformis.

CC. Beweglicher Finger am Oberrand ohne scharfen Kiel, mit mehreren Längsreihen von Körnern. Dactylus des 3. linken Beines mit einer Längskante auf der Aussenfläche.

D. Propodus des 3. linken Beines mit 2 scharfen Kanten am Oberrande.

DD. Propodus des 3. linken Beines mit nur einer Kante am Oberrande.

P. pedunculatus ${ }^{1}$ ).

Amboina, 1 Ex. (SEMON coll.).

Verbreitung: Japan (de HaAN); Sandwich-Ins. (Dana); Australien (Miers): Port Jachson (Miers); Batjan (Mıers); Ceylon (MÜller, Verh. Naturf. Ges. Basel, 8, 2, I887, p. 472).

\section{Clibanarius longitarsis (DE HAAN).}

Pagurus clibanarius Krauss, Südafrik. Crust., 1843, p. 56.

Pagurus longitarsis de HaAX, Faun. jap. Crust. dec. sept., 1849, p. 211, pl. 50, fig. 3.

Clibanarius longitarsis (D. H.) Daxa, U. S. Expl. Exp., 1852, p. 464 . Strmpsox, Proceed. Acad. Nat. Sc. Philad., 1858, p. 247. Heller, Crust. Novara, 1865, p. 90. De Max, Arch. f. Naturg., 53, 1, 188̄, p. 441.

Pagurus (Clibanarius) longitarsis D. H. Hilgexdorf, v. d. Deckens Reisen, III, 1, 1869, p. 96. Hilgexdorf, Mon. Ber. Akad. Wiss. Berlin, 1878, p. 821.

Dar-es-Salaam, im Hafen, i3 Ex., Upanga-Riff, I ot (ORTMAnn coll.). er seltener.

Lebt in zahlloser Menge im Hafen von Dar-es-Salaan auf sandigem Grunde. Auf den Riften ist

Verbreitung: Zanzibar (Hilgendorf); Ibo (Hilgendorf); Natal (Krauss); Madagascar: Nossi Bé (Lexz und Richters); Nicobaren (Heller); Java: Pulo Edam und Noordwachter (DE Man); Philippinen: Mindanao (DANA); Liu-Kiu-Inseln (Stmpson); Japan (DE HAAN).

\section{Cliballarius virescens (KRAUSS).}

Pagurus virescens Krauss, Südafrik. Crust., 1843, p. 56, pl. 4, fig. 3. Clibanarius virescens (Kr.) DaxA, U. S. Expl. Exp., 1852, p. 466, pl. 29, fig. 6. Hetler, Crust. Novara, 1865, p. 90.

1) Synonymie des $P$. pedunculanss (HERBST).

Cancer pedunculatus HERBST, Krabb. u. Kirebs., III, 4, I799, p. 23, p1. 61, fig. I.

Pagurus pedunculatus (HBst.) Hilgfndorf, Mon. Ber. Akad. Wiss. Berlin, 1878, p. 815.

Pagurus varipes Heller, Sitz. Ber. Akad Wiss Wien, 41. I, 1861, p. 241. pl. 1, fig. 1, pl 2. fig. 2. 3 Kossuare, Ergebn. Reis Roth. Meer, II, IS80, p 75. DE Man, Not. Leyd Mus., 11. ISSO. p. 1S4. DE Max, ibid. III. I881, p. I29. DE Man, Arch. f. Naturg. 53, 1. 1887, p. 436 .

Rothes Mleer, Hozambique, Java, Moluccen. 
Hilgexdorf, v. d. Deckens Reisen, III, 1, 1869. p. 95. Hilgexdorf, Mon. Ber. Akad. Wiss. Berlin, 1878, p. 821, pl. 3, fig. 11. DE MAx, Journ. Linn. Soc. Zool.. 22, 1888, p. 247.

Kilwa, Amanabank, I Ex., Dar-es-Salaam, im Hafen, I Ex. (Ortuaxx coll.).

Verbreitung: Zanzibar (Hılgexdorf); Mozambique (Hilgendorf); Ibo (Hilgendorf); Natal Krauss); Mergui-Inseln (DE MAN); Nord-Borneo: Balabac-Strasse (DANA); Sulu-See (DAna); Hongkong (HELLER); Fidji-Inseln (DANA).

\section{Calcinus herbsti DE MAN.}

Tgl. ORtMarx, Zool. Jahrb.. VI, 1892, p. 292.

Kilwa, am Strande, Io Ex., Dar-es-Sala m, Ras Chokir, 4 Ex. (Ortuaxs coll.).

Findet sich an felsigen Stellen des Strandes in Wasserlöchern, oft auch an bei Ebbe ganz trocken werdenden Plätzen in grosser Menge. Am Ras Chokir waren die Wandungen der von der Brandung ausgehöhlten Strudellöcher dicht mit Exemplaren dieser Art bedeckt.

Verbreitung: Von Ost-Afrika bis zu den Paumotu- und Sandwich-Inseln.

\section{Calcinus grimardi (MILNE-EDWARDS).}

Tgl. Ortuaxi, 1. c. p. 294.

Mein Exemplar zeigte im Leben folgende Farbenvertheilung: Vorderer Theil des Cephalothorax blassblau. Aeussere Antennen gelb. Augenstiele in der oberen Hälfte azurblau. Die übrigen Theile, die aus der Schneckenschale heraussehen (untere Hälfte der Augenstiele und Beine) dunkelbraun mit einzelnen weissen Punkten. Krallen vor der Spitze weiss, ebenso die Scheerenfinger.

Dar-es-Salaam, Upanga-Riff, I Ex. (OrTMani coll.).

Verbreitung: Von den Nicobaren über die Sunda-Inseln bis zu den Liu-Kiu-Inseln und Tahiti. -- Von Ost-Afrika bisher noch nicht bekannt.

\section{Dioyenes ruame HELLER.}

Heller, Crust. Novara, 186כ̄. p. 83, pl. 7, fig. 2. de Max, Journ. Linn. Soc. Zool., 2.2. 1888, p. 236. WALker, ibid. 20,1890 , p. 113 , pl. 8, fig. 6,7 .

Ton den mir vorliegenden Exemplaren stimmen nur zwei vollkommen mit $D$. avarus überein: es sind dieses das grösste von Dar-es-Salaam und das Exemplar von der Thursday-Insel. Die übrigen von Dar-es-Salaam sind sämmtlich kleiner (theilweis mit Eiern) und weichen in der Bildung der grossen Scheere insofern ab, als der Carpus nicht so auffallend verlängert und die Hand kürzer und gedrungener ist. Dass letzteres wahrscheinlich ein Merkmal jüngerer Exemplare ist, hat schon DE MAN ausgesprochen. Alle Exemplare stimmen darin überein, dass die grosse Scheere unbehaart und fein granulirt ist, und dass die Hand auf der Aussenseite eine kurze, stumpfe Längsleiste trägt. Letztere scheint für die Art charakteristisch zu sein.

Dass alle meine Exemplare zusammen gehören, ist jedenfalls unzweifelhaft, da ich sie sämmtlich an einem Tage, an derselben Localität und unter denselben Bedingungen sammelte.

Thursday Is land, I Ex. (SEHox coll. .

Dar-es-Salaam, im Hafen, 23 Ex. (Ortuan coll.).

Leben bei Dar-es-Salaam auf sandigem Grunde, dicht am Strande und sitzen meist in Nassa arcularia L. Ihre Bewegungen sind lebhaft und schnell.

Verbreitung: Mergui-Inseln (DE MAN); Nicobaren (Heller); Singapur (TValker).

\section{Eupagums hivtimanus Mers.}

Pagurus hirtimanus Gray. List specim. Crust. Brit. Mus., 1847, p. 60 (nomen nudum).

Eupagurus japonicus = hirtimanus (TH.) Mrers, Ann. Mag. N. H. 15), T, 1880, p. 375, pl. 14, fig. 6, 7.

Eup. hirtimanus (TH.) ve Max, Arch. f. Naturg., 53, 1, 1887, p. 426 und Not. Lejd. Mus., NII, 1890, p. 107.

Der Art-Name hirtimunus wurde als nomen nudum publicirt und zwar von Gray, richt von IVHITE. GRAY sagt zwar 1. c. p. IV, dass AD. WHITE mit grosser Sorgfalt die Species besagter Liste bestimmt hat etc., doch ist die Arbeit unter dem Namen von J. E. Grar erschienen, vgl. p. VIII. Miers constatirt die Identität des hirtimanus mit seinem vermeintlichen juponicus, und sagt ausdrücklich, wenn der letztere vom echten japonicus verschieden sein sollte, so solle der alte Name von IVHite (recte GRAY) adoptirt werden. Da nun dies thatsächlich der Fall ist, so ist der von Mites rorgeschlagene Name hirtimanus anzuvenden und Miers als Autor desselben anzusehen.

Amboina, 2 Ex. (Semox coll.).

Verbreitung: Philippinen (Gray, Miers); Carolinen: Ponapé (de Man); Amboina (DE Man); Neu-Guinea: Kaiser Wilhelms-Land (Mus. Strassburg); Fidji-Inseln (M1ERs. 
Familie: Coenobitidae.

Coenobita clypentus (HERBST).

Tgl. Ortmax, Zool. Jahrb., TI, 1892 , p. 316.

Amboina, $2 \delta$ I $q$ (SEMON coll.).

Dar-es-Salaam, I J (ORTMAN coll.).

Verbreitung: Ost-Afrika bis Tahiti. - Ton Amboina und Zanzibar schon angeführt.

Coenobita rugosus MILNE-EDWARDS.

Tigl. Ortusx, 1. c. p. $31 \%$.

Neu-Guinea, 3 Ex., Amboina, I $q$ Sexon coll. .

Dar-es-Sala am, 2 (ORTMANx coll.).

Terbreitung: Rothes Meer bis Natal, Sydney, Japan und Paumotu-Ins.

Coenobita commessus MILNE-EDwards.

Tgl. Ortmañ, l. c. p. 318.

Neu-Guinea, 2 Ex., Amboina, I jur. (Semon coll.).

Lindi, I juv., Kilwa, 0 Ex., Dar-es-Sala a m, viele Ex. ORTMAns coll.).

Verbreitung: Ost-Afrika über die Sunda-Inseln bis Japan. - Von Amboina und Neu-Guinea noch nicht erwähnt.

\section{Coenobita perlotus MiLne-EDwards.}

Vgl. Ortuaxi, l. c. p. 319 .

Dar-es-Salaam, I + ORTMAN coll.).

Verbreitung: Indo-pacifisches Gebiet: von Ost-Afrika bisher noch nicht bekannt, die nächsten Fundorte sind: Seychellen (Richters; Amiranten lMiers; Mauritius (Miers, OrtMann:.

Tgl. Ortulis, l. c. p. 319

\section{Birgus latro LINÉ).}

Amboina, 4 Ex. SEMon coll. .

Verbreitung: Inseln des Indo-pacifischen Gebietes, rom Afrikanischen, Indischen und Australischen Festlande noch nicht bekannt.

Komoren (Hilgendorf; Glorioso-Gruppe (Miers; Mauritius (Hoffmann, Ortmann); Amboina (DE MAN); Ternate (Henderson); Liu-Kiu-Inseln (DE HAAN; Fidji-Ins. (Mrers); Paumotu- und TokelauArch. (DANA).

Familie: IIppidae.

Remipes testulimarils LATREILLE.

Tgl. Ortmasx, l. c. p. 537 .

Amboin a, 7 Ex. (SEmon coll..

var. denticulatifrons WHITE.

A mboina, 3 Ex. (Semon coll.).

V erbreitung: Ueberall im tropischen Indo-pacifischen Gebiete.

Familie: Dynomenidae.

Tgl. Ortunis, 1. c. p. 543 !

\section{Dynomene hispiala DESMAREST.}

A mboina, I adult. (SEMON coll.)

Verbreitung: Mauritius MilNe-Edwards:; Amboina (DE MaN); Liu-Kiu-Ins. OrtManN:; NeuCaledonien (A. Mline-Edwards'; Sandwich-Inseln A. Milne-Edwards).

I) Daselbst fehlt das Citat: DE MAx, Arch. f. Naturg.. 53, 1, IS8\%, p. 408 .

Jenaische Denkschriften VIII. 
Familie: Dromiidae.

\section{Dromilia unillentat (RÜPPELL).}

Dromia unidentata Rüppell, 24 Art. Krabb. Roth. Meer., 1830, p. 16, pl. 4, fig. 2. Mrilxe-Edwards, Hist. Nat. Crust., II, 1837, p. 178. Hrlgendorf, Mon. Ber. Ak. Wiss. Berlin, 1878, p. 813.

Dromidia unidentata (Rpp.) Kossmanx, Zool. Erg. Reis. Roth. Meer., 2, 1880, p. 67. de Max, Journ. Linn. Soc. Zool., 22,1888, p. 207, pl. 14, fig. 4, 5 (var.).

Länge des Cephalothorax $20 \mathrm{~mm}$, Breite $19 \mathrm{~mm}$; Oberfläche stark gewölbt, dicht filzig behaart, unter den Haaren glatt, nur die Cardiacalgegend nach vorn durch zwei Gruben begrenzt. Auf den Branchialgegenden je zwei kleinere Gruben. Stirn tief eingeschnitten, der mittlere Lappen ganzfehlend, die seitlichen dreieckig, vorragend. Supraorbitalzahn etwa halb so gross wie die letzteren. Aeussere Orbitalecke stumpf, Zahn des Unterrandes der Orbita dreieckig, von der äusseren Ecke durch eine tiefe, spaltförmige Fissur getrennt.

Vorderseitenrand ohne $Z$ ähne, erst dicht hinter der Cervicalfurche ein kleiner Zahn. Von dieser Furche läuft nach vorn eine sehr ausgeprägte, stumpfe, bogenförmige Kante, die sich vorn etwas nach unten neigt und den Inframarginalwulst bildet. Darunter ein stumpfer, wulstartiger Höcker (Suprasuturalwulst). Die Sutur selbst ist furchenförmig.

Diese Art ist an der Bildung der Stirn und des Vorderseitenrandes leicht zu erkennen. Da mir nur ein ơ vorliegt, kann ich über die Gattungszugehörigkeit nichts aussagen: nach KossıAxi gehört sie zu Diomidia.

Dar-es-Sala am, Chokirbank, I J , mit einer Weichkoralle auf dem Rücken (OrTMANx coll..

Verbreit ung: Rothes Meer (RÜPPell, Kossuaxi); Ost-Afrika Bianconı); Ceylon Müller, Verh. Nat. Ges. Basel, S, 2, 1887, p. 472); Mergui-Inseln (DE MAN, var.).

\section{Cryptorlvomiu lamellatu nor. spec. Tafel II, Fig. S.}

Cephalothorax glatt, im Umriss 5-seitig. Stirn dreizähnig, mittlerer Zahn schräg abwärts gerichtet, alle drei spitz. Die seitlichen setzen sich in einen oberen Augenhöhlenrand fort, der nach aussen in einer spitzen Ecke endigt. Vorderseitenrand mit zwei Zähnen, von diesen ist der erste von der äusseren Superciliarecke durch eine tiefe Bucht getrennt und springt breit-lamellenartig vor nach vorn ist er abgestutzt. Der zweite Zahn ist dreiechig, klein. Darauf folgt noch, gleich hinter der undeutlichen Cervicalfurche, ein ganz kleiner dritter Höcker. Unterer Augenhöhlenrand ohne Höcker. Inframarginalwulst und Suprasuturalwulst nur durch je einen länglichen Höcker angedeutet.

Scheerenfüsse schwach. Merus scharf dreikantio. Carpus aussen und innen mit einem fast flügelartigen Kiel, dazwischen auf der oberen Fläche etwas lnotig. Hand länglich, mit $2-3$ aus Knoten gebildeten Längswülsten. Scheerenfinger nur an den Spitzen sich berührend, daselbst mit ineinander greifenden Zähnen. Das 2. und 3. Bein zeigt auf dem Merus 3 fast flügelartige Kiele, die Carpen sind flügelig dreikantig, die Propoden vierkantig. Alle Kanten sind etwas gewellt, aber nicht eigentlich linotig, sondern scharf. Die Krallen tragen am unteren Rande 4 kleine, hornfarbige Dörnchen. Die beiden hinteren Beinpaare zeigen weniger scharfe Kiele, besonders fehlen die des Carpus und Propodus.

Abdomen der $q$ breit. Letztes Glied fast queroval.

Der ganze Körper ist kahl: an der Spitze der Krallen des 2. und 3. Beinpaares stehen einige Borsten.

Sternalfurchen getrennt, bis zum 2. Beimpaar reichend, Gaumen mit Leiste, Scheerenfinger mit weissen Spitzen: also zu Cryptodromia gelıörig.

Thursday Island, I q (SEMon coll.).

Cryptodromia pentagonatis HILGENDORF.

Hilgendorf, Mon. Ber. Akad. Wiss. Berlin, 1878, p. 814, pl. 2, fig. 1, 2.

Die kurze Beschreibung bei HıLGENDORF genügt völlig, um die Art wiederzuerkennen. Dennoch glaube ich, wird es nicht überflüssig sein, eine nochmalige Beschreibung zu geben, da mir auch das bisher unbekannte $\delta$ vorliegt.

Zunächst muss ich bemerlien, dass die Sternalfurchen meines sehr kurz und weit getrennt sind und sich nur luis in die Höhe der $q$ Genitalöffnung erstrecken: ein. Terhältniss, das von dem der übrigen Arten der Gattung, wo sie bis zum 2. Beinpaar reichen, etwas abweicht.

Cephalothorax fünfseitig. Stirn dreizähnig, der mittlere Zahn gut entwickelt. Vorderseitenrand nur mit einem Zahn, vor diesem concav, dahinter fast gerade. Sonst ist nur der Zahn des Hinterseitenrandes, dicht hinter der Cervicalfurche, vorhanden, aber klein. Infraorbitalzahn mit einem kleinen Nebenhöcker. Zwischen diesem und dem Seitenzahn ein kräftiger Höcker auf dem lnframarginalwulst. Suprasuturalwulst ohne Höcker. 
Abdomen der $\delta$ mit lang dreieckigem Endgliede, welches durch zwei stumpfe, seitliche, basale Lappen eine pfeilförmige Gestalt bekommt. Scheeren des ठ kräftiger, sonst aber ähnlich denen des \%.

Dar-es-Sala a m, Upanga-Riff, I $\delta$ I $¥$ (ORTMANs coll.).

Verbreitung: Ost-Afrika: Ibo (Hilgendorf).

\section{Psendodromin latens Stimpson.}

Strupsox, Proceed. Acad. Nat. Sc. Philadelphia, 1858, p. 78. Hexdersox, Chall. Anomur., 1888, p. 16, pl. 1, fig. 8.

Die Sternalfurchen der $q$ reichen bis zum 2. Beinpaar, nähern sich nach vorn und endigen in einem einzigen Höcker (vergl. Hexdersor).

Cap: Port Elisabeth, I $\delta 2$ (Mus. Strassburg).

Verbreitung: Cap: Simons Bay, Io-i2 Fad. (Stimpson, Henderson).

\section{Familie: Calappidae.}

\section{Mursia cristata MILNE-EDWARDS.}

Mursie mains en crête Demarest, Consid génér. Crust., 1825, pl. 9, fig. 3.

Mursiu :\%. Latreille in Crvier: Regn. anim., 2- ed., IV, 1829, p. 39.

Mursia cristiata (sic!) MrLxe-Edwams. H. Nat. Crust., II, 1837. p. 109

Mursia cristata Latr. Milse-Edwards, in: Curter, Atl. Regn. anim., pl. 13, fig. 1.

Mursia cristimana de Hadx, Faun. Japon. dec. quarta, 1839, p. 73. Kracss, Südafrik. Crinst., 1813 , p. 52. Mlers, Chall. Brach., 1886, p. 291

Mursia cristata Daxa, U. S. Expl. Exp, 1852, p. 394. STuder. Abh. Ak. Wiss. Berlin 188?. T. 15.

Cryptosoma orientalis ADAws et White. Zool. Toy. Samarang. Crust., 1848, p. 62, pl. 13, fig. 4.

Cap: Port Elisabeth. I (Mus. Strassburg).

Verbreitung: Süd-Afrika (Milne-Edwards); Cap (DE HAax, Kralss, Dana, Miers), Tafelbai

(Studer). - Adans u. White geben Eastern Seas an, was sehr in Zweifel zu ziehen ist.

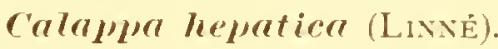

Tgl. OrtMaxx, Zool. Jahrb., TI, 18:2, 1. 568.

Amboina, 2 Ex. (SEMoN coll.).

Kilwa, Amanabank, I Ex., Dar-es-Salaam. im Hafen, zahlreiche Ex. (Ortaxx coll.).

Verbreitung: Rothes Meer bis Natal, Liu-Kiu-Ins., Sydney, Neu-Seeland, Tahiti und Sandwich-

Inseln. Im Mus. Strassburg befindet sich auch ein Exemplar von Japan (wahrscheinlich südliches). - Eine ganz allgemein verbreitete und charahteristische Art in der Indo-Pacifischen Region.

\section{Familie: Matutidae.}

Watutu victrix FABRICIUS.

var. tylica ORTMANN (M. victrix MIERS).

Vgl. ORTMANx, 1. c. 189.2, p. $5 \bar{i} 1$.

Dar-es-Sala am, im Hafen, zahlreiche Exemplare (ORTMANN coll.).

Verbreitung: Rothes Meer bis Natal und Ost-Australien.

Tgl. Ortanax, 1. c. p. 573.

var. bamlisi Miers.

Amboina, I 2 ? (SEMON coll.).

bekannt.

Verbreitung: Bisher nur von den Ost-Asiatischen Meeren, China-See, Java bis Ost-Australien

Familie: Lencosiidae.

\section{Oreophorks myosus Stimpson.}

Vgl. Ortuxx, Zool. Jahrb., TI. 1892, p. 57j

Thursday Island, I 2 SeMox coll. .

Verbreitung: Süd-Japan: Kagoshima Ortanxis); Liu-Kiu-Inseln (Stmpson; Cochinchina

(A. Milve-Edwards; Australien: Port Denison Haswell; Neu-Caledonien A. Milne-Edwards). 
Ixa cylindives (FABRICIUS).

I. canaliculata Leach, Mule-Enwards, H. N. Cr., II, 1837, p. 135.

1. inermis Leach, Milne-EDwards, ibid.

1. canaliculata Leach, Minne-Enwards, in Curifr, Atl. Regn. anim., pl. 21, fig. 1.

I. megaspis Adums et Whit, Zool. Voy. Samarang, 1848, p. 55, pl. 12, fig. 1.

I. cylindrus (FABR.) BeLL, Trans. Linn. Soc., 21, 1855 , P. 311.

I. edwardsi Luc. A. Nilne-Edwarys, Annal. Soc. entomol. France (4), V, 1865, p. 156, pl. 6, fig. 1.

Thursday Island, I $\delta$ (SEMON coll.).

$\mathrm{K}$ il wa, auf der Rhede, 8-IO $\mathrm{m}$, ein Rückenpanzer (ORTMANN coll.).

Verbreitung: Indischer Ocean (Bell); Zanzibar (A. Milne-Edwards); Mauritius (MILne-EdWARDS); Borneo (Adaws et White); Philippinen (Adans et White).

Vgl. Ortmani, 1. c. p. 582.

$$
\begin{aligned}
& \text { Myra fugax (Fabricius). } \\
& \text { iar. coalita Hilgendorf. }
\end{aligned}
$$

Amboina, 1 o spur. (Semon coll.).

Kilwa, auf der Rhede, 8-I0 m, I $q$ spur. (ORTMANn coll.).

Verbreitung: Rothes Meer und Ost-Afrika bis Japan und Neu-Caledonien. Die var. coalita ist von Zanzibar und Japan bekannt.

\section{Mypa affinis BELL.}

Belt, Trans. Linn. Soc. London, 21, 1855 , p. 296, pl. 32, fig. 2. Srmpsus, Proceed. Acad. Nat. Sc. Philadelphia, 1858, p. 160. Haswell, Catal. Austral. Crust., 1882. 1. 121. Mikrs. Rep. Zool. Coll. Alert, 1884, p. 250. Miers, Chall. Brach., 1886. p. 315.

Thursday I sland, 4 spur. (SEMox coll.).

Verbreitung: Japan: Kagoshima (Strupson); Philippinen (Bell); Torres-Strasse: Thursday Isl. (Miers); Queensland: Cap Grenville (Haswell), Port Denison (Miers).

\section{Nursia ypsilon nor. spec. Taf. Il, Fig. 7.}

Cephalothorax fast fünfseitig, von einem kielförmigen, scharf granulirten Rand rings umzogen. Unter dem vorderen Theil des Vorderseitenrandes läuft auf der Pterygostomialgegend ein ähnlicher, granulirter Kiel, der sich ungefähr in der Mitte des Vorderseitenrandes mit dem oberen, von den Augen ausgehenden Kiel vereinigt: an der Vereinigungsstelle wird eine kleine Ecke gebildet. Ebenso liegt in der Mitte des Hinterseitenrandes noch eine kleine Ecke. Von letzterer zieht sich auf der Oberfläche des Cephalothorax über die Branchialgegend nach vorn ein grob granulirter Wulst, der sich nach vorn in zwei Aeste theilt. Mitte der Oberfläche des Cephalothorax grob granuliert: es lassen sich drei Längslinien gröberer Körner unterscheiden, die sich nach hinten auf der Cardiacalgegend vereinigen. Dahinter liegt auf der Intestinalgegend eine etwa rhombische Gruppe gröberer Körner. Sonst zeigt die Oberfläche des Cephalothorax nur ganz feine, zerstreute Granulationen.

Stirnrand quer abgestutzt. Augenhöhlen nicht von den Antennengruben getrennt. Exopodit des 3. Maxillarfusses mit gebogenem Aussenrande.

Merus, Carpus und Palma der Scheerenfüsse fein granulirt. Merus etwa cylindrisch. Palma und Finger etwas comprimirt. Die übrigen Beine sind glatt, mit ziemlich langem, fast geradem Dactylus.

Abdomen des 3 lang-dreieckig, 3. bis 6. Segment verwachsen, am distalen Ende mit einem Höcker. Abdomen des $q$ fast kreisrund, 4. bis 6. Segment verwachsen, 7. Segment klein, länglich, etwa doppelt so lang wie breit.

Länge des Cephalothorax des grössten Exemplares (7): $7 \mathrm{~mm}$.

Steht der N. ablreviata BeLL (Trans. Linn. Soc., 21, 1855, p. 308, pl. 34, fig. 5) aus dem Indischen Ocean und der $N$. rubifera MüLler (Terh. Naturf. Ges., Basel, 8, 2, 1887, p. 480, pl. 4, fig. 4) von Ceylon im Unriss des Cephalothorax am nächsten, unterscheidet sich aber durch die Sliulptur der Oberfläche.

Thursday Island, I $\delta 2+$ (SEMON coll.).

Philyra platycheire DE HAAN.

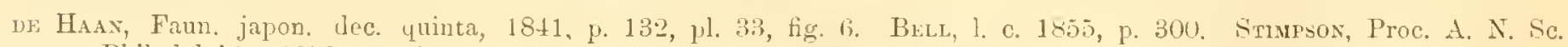
Philadelphia, 1858, p. 160. 1) Max; Journ. Linn. Soc. Zool., 22, 1888, p. 201.

Diese Art charakterisirt sich durch folgende Merkmale:

Cephalothorax oben glatt, olme Köner, fein punktirt. Vorderseitenrand nicht ausgeprägt, durch einen kleinen Höcker angedeutet. Pterygostomialkante in den Hinterseitenrand übergehend, gekörnt, die Körner nach hinten zu verschwindend. 
Merus der Scheerenfüsse an der Basis und an Vorderrande granulirt. Carpus und Hand glatt, schmal. Finger an den Schneiden zahnlos, der unbewegliche bärtig.

Dar-es-Salaam, Ras Cholir, im Sande, I d (ORTuann coll.). (DE MAN).

Verbreitung: Japan (DE HAAN); Hongkong (Stimpson); Philippinen (BelL); Mergui-InseIn

\section{Leueosia perluta DE HAAN.}

Tgl. Ortmane, Zool. Jahrb., VI, 1892 , p. 584.

Amboina, I $q$ spur.; Thursday Island, I $q$ I $q$ spur. (Semon coll.).

Verbreitung: Grosse und kleine Sunda-Inseln, Philippinen.

\section{Lelreosia maryaritacea BELL.}

BkLL, Trans. Linn. Sc., 21, 1855, p. 288, pl. 30, fig. 8.

Vollkommen mit Beschreibung und Abbildung übereinstimmend.

Kilwa, Rhede, S-10 m, I t spur. (OrTMANn coll.).

Ocean an

Verbreitung: Ein genauerer Fundort war bisher nicht bekannt: BeLL giebt den Indischen

\section{Familie: IIymenosmmidae.}

\section{Hymcuosoma orbiculare Desmarest.}

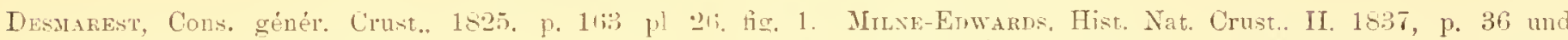

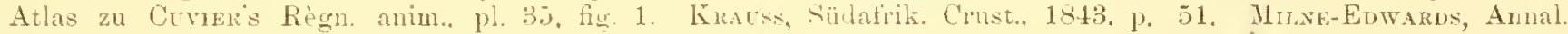
Sc. Nat. (3) Zool., 20, 1853 , 1). 22.2. Srmpros, Proceed. Acad. Sc. Philadelphia, 1858, p. 108. Miers, Chall. Brach, 1886, p. 280

Cap: Port Elisabeth, I 82 q (Mus. Strassburg).

Verbreitung: Cap der guten Hoffnung (Desmarest, Milne-Edwards) : Tafelbai (Krauss) SimonsBai (Stimpson, Miers).

\section{Familie: Inachidat.}

Stenorh!mehus brevis nor. sper. Tafel I, Fig. 5.

Cephalothorax fast dreieckig, vorn verschmälert, wenig gewölbt. Rostrum aus zwei dicht an einander liegenden Dornen bestehend, spitz dreieckig, kurz, kaum halt so lang als der freie Theil der Stiele der äusseren Antennen. Praeoculardorn und Postoculardorn fehlend. Augen frei vorragend, Stiele an der Spitze über der Cornea mit einem lileinen Dorn. Das feste Stielglied $12+3)$ der äusseren Antennen, sowie das Epistom ohne Stacheln.

Gastricalregion mit drei kleinen Höckern, der hintere etwas grösser als die vorderen. Hepaticalgegend mit'zwei kleinen Höckern. Cardiacalgegend mit einem stumpfen Höcker, der etwas höher ist, als der hintere Höcker der Gastricalgegend. Branchialgegenden gewölbt, aber ohne Höcker oder Dornen.

Scheerenfüsse ziemlich kräftig. Hand etwas geschwollen, wie die übrigen Pereiopoden etwas behaart. Letztere lang und schlank, Krallen der 2. und 3. lang, fast gerade, der der 4. und 5. kürzer und gebogen.

Durch die Kürze des Rostrums und das gänzliche Fehlen von Stacheln auf Epistom und festem Stielglied der Antennen von allen bekannten Arten unterschieden.

Kilwa, Rhede, $\mathrm{S}$-IO $\mathrm{m}$, in Seegras, I o (ORTIAN coll.).

\section{Achacus reffillis Mers.}

Miers, Rep. Zool. Coll. Alert, 188t, p. 188. De Max, Arch. f. Naturg. 53, 1, 1886, p. 218.

Auf der Cardiacalregion zeigen beide mir vorliegenden Exemplare einen an der Spitze schwach 2-teiligen Höclier, dahinter steht, wie es DE MAN beschreibt, ein ganz kleiner Höclier. Bei dem einen (kleineren) Exemplar ist die Magengegend so, wie es DE MAN beschreibt: bei dem anderen trägt sie aber im medianen hinteren Theil einen ziemlich spitzen, deutlichen Höcker, der fast so hoch ist, wie der Höcker der Cardiacalgegend. Ich lege auf diesen Unterschied kein Gewicht, da die Ausbildung der Höcker des Cephalothorax auch bei anderen Arten (vergl. tuberulatus Miers bei OrTmann, Zool. Jahrb., VII, I803, p. 34) variirt. Die Augenstiele haben in der Mitte des vorderen Randes den für diese Art charakteristischen Höcker. 
Thursday Island, 2 (SEMON coll.).

Verbreitung: Ost-Australien: Port Jackson (Miers), Port Denison, Moreton-Bay (Miers); TorresStrasse: Thursday Island und Prince of Wales Channel (Miers); West-Australien: Shark-Bay (Miers); Java: Insel Noordwachter (DE MAN).

\section{Paratymolus pubescens MiERs.}

Vgl. Ortmanx, Zool. Jahrb., VII, 1893, p. 35.

$P$. bituberculatus HASWELL unterscheidet sich nur durch den Dorn der Palma vor dem beweglichen Finger. Die mehr dreieckige Form der Scheere in der Abbildung bei Haswell (Ann. Mag. N. H. (5), V, I880, pl. I6, fig. I, 2) ist vielleicht als Sexualcharakter anzusehen, da Haswell ein o abbildet, während Miers nur ein $q$ besass. Die feinen Dornen am Merus der Scheerenfüsse sind auch bei japanischen Exemplaren vorhanden.

Kilwa, Rhede, 8-io m, i 8 I q (Ortmann coll.).

Verbreitung: Japan: Matoya (Miers), Kagoshima und Tanagava (Ortmans). - P. bituberculatus kommt in der Torres-Strasse und in Queensland vor.

\section{Camposcia retus゙ LATREILLE.}

Vgl. Ortmaxx, Zool. Jahrb., TII, 1893, p. 35. A mboina, I $q$; Thursday Island, 28 (SEmon coll.).

Verbreitung: Vom Rothen Meer und Ost-Afrika bis zu den Fidji-Inseln.

\section{Gattung: Amacinetops MIERs.}

Miers, Ann. Mag. Nat. Hist. (5), IV, 1879. p. 3.

Diese Gattung gehört in die Verwandtschaft von Eucinetops Stimpson und Camposcia Latr. (vgl. Miers, Journ. Linn. Soc., I 4, I879, p. 644). Sie würde sich durch folgende Diagnose unterscheiden:

Cephalothorax länglich. Rostrum kurz, von zwei abgeflachten Dornen gebildet, die durch einen schmalen Spalt getrennt sind. Augen lang und schlank, gegen die Seiten des Cephalothorax zurücklegbar. Kein Praeorbitaldorn. Hinter den Augen zwei hintereinander stehende Postoculardornen. Keine untere Begrenzung von Orbiten. Festes Stielglied der äusseren Antennen etwa doppelt so lang wie breit, die beiden folgenden Glieder cylindrisch. Dritter Maxillarfuss mit am distalen Ende abgestutztem und schwach ausgerandetem Merus. Gehfüsse mittelmässig schlank.

Anacinetops stimpsoni MiERs. Tafel III, Fig. 2.

Eucinetops? stimpsoni MiER\%, ibid.

Ein mir vorliegendes $\delta$ glaube ich zu dieser Art bringen zu können. Wenn auch die Beschreibung bei Miers etwas kurz ist, so widerspricht keines der angegebenen Merkmale dieser Zugehörigkeit.

Cephalothorax fast birnenförmig, von der Spitze des Rostrums bis zum Hinterrande $20 \mathrm{~mm}$ lang. Oberseite convex, ohne Dornen und Tuberkeln, nur etwas runzelig an den undeutlichen Grenzen der Regionen. Rostrum schwach abwärts geneigt, etwa bis zur Hälfte in zwei abgeflachte und spitze Dornen gespalten. Oberer Augenhöhlenrand ohne Praeorbitaldorn, hinter den Augen in einen kleinen Lappen vorspringend. Dahinter stehen zwei weitere, etwas abgeflachte, stumpfe Dornen, deren hinterster dem Postoculardorn homolog ist. Augen schlank, wenn yegen die Seiten zurückgelegt, bis unter den Postoculardorn reichend. Festes Stielglied der äusseren Antennen etwa doppelt so lang wie breit, am distalen Ende mit zwei kleinen Höckern, einem an der äusseren, einem an der inneren Ecke. Von einem unteren Orbitalrand ist keine Spur vorhanden.

Merus des 3. Maxillarfusses am distalen Ende abgestutzt und schwach ausgerandet.

Scheerenfüsse des $\delta$ ziemlich schwach und glatt. Palma etwas comprimirt, Finger dünn und spitz, an den Schneiden undeutlich gezähnt, an der Basis ein wenig klaffend. Die folgenden Pereiopoden sind mittelmässig, nehmen von vorn nach hinten etwas an Länge $a b$, und sind nur wenig länger als der Cephalo. thorax (2. Pereiopoden $27 \mathrm{~mm}$, fünfte $22 \mathrm{~mm}$ ).

Abdomen des $\delta$ aus sieben getrennten Gliedern bestehend

Körper und Beine mit zahlreichen, steifen, hakenförmigen Haaren besetzt und mit einem dichten Filz von Algen, Bryozoen und Schlamm bedecht.

Thursday Island, I $\delta$ (SEMON coll.).

V'erbreitung: Nordost-Küste Australiens (MIERs). 
Tegl. Ortmañ, 1. c. 1893, p. 40.

Iruenir proters DE HAAN.

Thursday Island, I \& (SEMon coll.).

Verbreitung: Japan (DE HAan): Kadsiyama (Ortuaxi); China (Adas et White); Philippinen (Adas et White); Torres-Strasse (HAswell): Thursday Island (Miers), Cap York (Miers); Queensland (Mrers); Norfolk-Inseln (Miers); Ceylon (MÜLler).

\section{Huenia grumblieri A. HLXE-EDWARDS.}

H. grandidieri Mnxe-EDWAids, Annal. Soc. entom. France 4, T, 1515, p. 143, pl. 4, fig. 2.

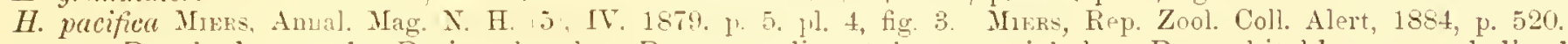

Durch das an der Basis schmalere Rostrum, die stärker entwickelten Praeorbitaldornen und die drei Höcker auf der Gastricalregion von $H$. protcus unterschieden.

Merus der Pereiopoden distal mit einem stumpfen Dorn oder Höcker. Scheeren des $\delta \mathrm{kräftig.}$ Palma etwas comprimirt, Finger viel kürzer als die Palma, klaffend, nur an der Spitze sich berührend und daselbst mit ca. + in einander greifenden Zähnen. Beweglicher Finger nahe der Basis mit einem rechteckig abgestutzten Höcker.

Das von Kilwa zeigt ein etwas längeres Rostrum als die übrigen Exemplare, geringer entwiclielte vordere Lappen des Seitenrandes und schwächere Höcker der Oberfläche. Es stimmt in der äusseren Körperform gut mit der Abbildung der $H$. prifer, welche letztere offenbar mit gramdidieri identisch ist.

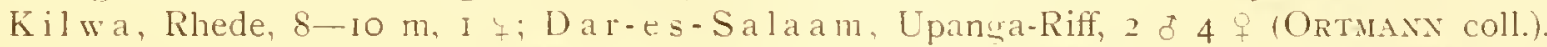

Verbreitung: Zanzibar (A. MilNe-EDwards); Seychellen (Miers); Fidji-Inseln (Miers).

\section{Henuethills momorerus (LATREILLE).}

Tgl. Ortmixi, l. c. p. 41.

Dar-es-Sal a am, Upanga-Riff, I I + (ORTuANa coll.).

Verbreit ung: Vom Rothen Meer, Zanzibar und den Mascarenen bis zu den Samoa- und SandwichInseln.

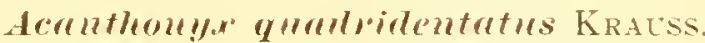

Eratss, Südafrik. Crust, 1843. p. 4: pl. 3. fig. 7 .

Mit der Beschreibung und Abbildung gut übereinstimmenć nur stehen auf der vorderen Branchialregion jederseits noch zwei ganz kleine Höckerchen.

Dar-es-Sala am, Upanga-Riff, I d (OrTMANí coll.).

Terbreitung: Natalküste (Krauss).

\section{Acamthonyex Iputatus MILNE-EDWARDS.}

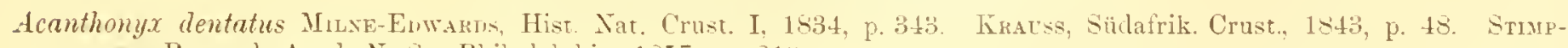
sin, Proceed. Acal. N. So. Philadelphia. 185\%. 1\% 219.

Dehaanius acanthopus Machear, Anuulosa in: Surru. Zool. S. Afric., 1838, p. 58, pl. 3.

Deharnius dentatus Mifis. Chall. Brach., 1886, 1. 39.

Ich sehe den Grund nicht ein, weshalb VIERs diese Form als Dehamius MAcLEAy von der Gattung Acanthonyx trennen will.

Cap: Port Elis abeth, 6 Ex. (Mus. Strassburg).

küste (KRAuss).

Verbreitung: Cap der guten Hoffnung (Milwe-Enwards): Simons-Bay (Stimpson, Miers); Natal-

\section{Gattung: Pseudomiriphe Heller.}

Miers (Journ. Linn. Soc. Zool., I4, 1879, p. 601) stellt diese Gattung in die Unterfamilie Micippinae der Familie Majidue. Nach der Gestalt der Augenhöhlen kann sie aber in dem System von MıEs nur zur Familie Inachidac gehören, und zwar entweder zur Tnterfamilie Lnachinae, und dann in die nächste V'erwandtschaft von Halimus (vielleicht hat HAswell diese Verwandtschaft richtig erkannt, wenn er eine von ihm beschriebene, mit Zweifel hierher gehörige Form als Microhulimus in eine Untergattung von IIalimus bringt); oder man stellt diese Gattung noch besser in die Unterfamilie Stenocinopinae, nur ist bei ihr der Praeoculardorn nicht so stark entwickelt. Da das System von Miers ein ganz künstliches ist, so halte ich es für überflüssig, zu versuchen, dieser Gattung in demselben eine feste Stellung anzuweisen: auf keinen Fall gehört sie aber in die Familie der Mrujulıe. und mit Nicipue hat sie gar nichts zu thun.

Die drei bekannten Arten lassen sich in folgender Weise zusammenstellen: 
a. Praeoculardorn als stumpfer Dorn gut entwickelt, an seiner Basis oben ohne besonderen Höcker sondern nur wulstig. Gastricalgegend in der Mittellinie nur mit zwei deutlichen Höckern.

Ps. nodosa Heller.

aa. Praeoculardorn nur als kleiner Höcker entwickelt, seine Basis bildet oben (zwischen den Augen) je einen deutlichen Höcker (Bord sus-orbitaire très-saillant et surmonté d'un gros tubercule A. M.-E.). Gastricalgegend in der Mittellinie mit vier deutlichen Höckern ${ }^{1}$ ).

b. Sternum an der Berührungsstelle der Segmente mit leicht granulirten, vorspringenden Kielen.

bb. Sternum ohne diese Kiele. Ps. tenripes A. M.-E. ${ }^{2}$ ). Ps. varians MiERs.

\section{Psendomicippe nodosa Heller.}

Heller, Sitz.-Ber. Akad. Wiss. Wien, 43, 1861, p. 303, pl. 1, fig. 3-6.

Bei dem vorliegenden $q$ sind die Rostralhörner, wie es HELler angiebt, fast senkrecht nach unten gerichtet, und sie entsprechen völlig der Abbildung. Bei den $\delta$ ist die Neigung lange niclut so stark, wie auch die Gastricalregion nicht so auffällig erhaben und gegen die Stirn zu weniger abschüssig ist. Auch sind die Rostralhörner bei den $\delta$ spitz, nur bei dem lileinsten Exemplar stumpflich.

Dar-es-Salaam, Upanga-Riff, 5 o I $q$ (ORTmann).

Verbreitung: Rothes Meer (Heller).

\section{Pscudomicime varians Mllers.}

Mifers, Amn. Mag. N. H. (5), IT, I879, p. 12, pl. 4, fig. 8. Mifers, Rep. Zool. Coll. Alert, 1884, p. 197. Mifre, Chall. Brach., 1886 , p. fis.

Wie bei der vorigen Art ist die Abschüssigkeit der Gastricalgegend nach vorn und die Neigung der Rostralhörner beim $\delta$ und $q$ verschieden. Diese Neigung ist bei meinen $q$ etwa so stark, wie bei den $\delta$ der vorigen Art, oder etwas schwächer, vgl. die Abbildung bei Miers, Fig. 8 a, bei den $\delta$ ist sie noch schwächer.

Miers vermuthet, dass Microlalimus deflexifrans Haswell (Catal. Austral. Crust., IS82, p. 7) von Port Jackson mit dieser Form identisch sei: auch ich möchte mich dieser Ansicht anschliessen, leider ist aber die Beschreibung bei HaswELL recht ungenau.

Thursday lsland, 3 ठ 3 \& (Semon coll.).

Verbreitung: Queensland: Port Denison; Torres-Strasse, Thursday Island; West-Australien: Shark-Bay (MıERS).

Familie: Hajidle.

\section{Egeria arachmoinles (Rumph).}

Vgl. Ortmann, Zool. Jahrb. VII, 1893, p. 48.

Thursday Island, ein selir schlechtes Exemplar (SEmon coll.).

Verbreitung: Von Indien (Coromandel) über die Ost-Asiatischen Inseln bis China, zu den Philippinen und Australien. Aus der Torres-Strasse von Haswell erwähnt.

\section{Maja squimado (RONDELET).}

\section{inu. capensis mor:}

Diese Form stimmt in der allgemeinen Körpergestalt völlig mit Exemplaren der M. squinado aus dem Mittelmeer überein, sie zeigt jedoch insofern eine gewisse Annäherung an M. vermucosı M.-E., als auf der Oberfläche des Cephalothorax zahlreiche gerundete, stumpfe Warzen an Stelle der kegelförmigen Stacheln der M. squinado stehen. Während aber bei $\boldsymbol{M}$. verrucosa aus dem Mittelmeer fast die ganze Oberfläche des Cephalothorax mit solchen stumpfen Warzen besetzt ist, finden sich bei der vorliegenden Form noch manche spitze, kegelförmige Dornen, z. B. auf den Branchialgegenden. Vor allen Dingen aber zeigt die var. capensis in der Mittellinie des Cephalothorax eine Reihe von hohen, liräftigen, spitzen Dornen, die bei $\boldsymbol{M}$. verrucosa nie in dieser Weise entwickelt sind, sich jedoch bei $M$. squinado finden. Aus diesem

I) A. Milne-Edwards sagt im Text mit 5 Hockern, seine Abbildung zeigt aber nur 4.

2) A. Milne-Enwards, Annal. Soc. entomol. France (4), V, 1865, p. 139, pl. 5, fig. 2. - Indischer Ocean. 
Grunde sehe ich auch die vorliegende Form als var. von squinado und nicht von verrucosa an. Jedenfalls ist sie eine vermittelnde Form zwischen beiden.

Der Fundort ist sehr auffällig. Seine Richtigkeit wird aber - abgesehen davon, dass die übrigen im Mus. Strassburg befindlichen Formen ron Port Elisabeth, die alle aus derselben Quelle stammen, durch. aus keinen Zweifel in Bezug ihres Herkommens aufliommen lassen - besonders noch dadurch verbürgt, dass sich auf den Exemplaren gewisse Bryozoën vorfinden, die für Süd-Afrika charakteristisch sind, wie Catenicella sp. und Onchoporella bombycina $L$.

Da diese Form sich eng an die beiden europäischen Arten anschliesst, die bisher ausserhalb des Mittelmeeres und der NW.-Europäischen Küsten noch nicht gefunden wurden, so wäre es interessant, wenn die letzteren sich auch an den West-Afrikanischen Küsten auffinden liessen, wodurch dann die Continuität des Verbreitungsgebietes von Europa bis Süd-Afrika hergestellt würde: ein Verhältniss, das nichts Auffallendes haben wïrde, da Formen mit einer derartigen Verbreitung schon bekannt sind.

Cap: Port El isabeth, 3 (Nus. Strassburg).

\section{Gattung: IIyrostemus.}

Versuch einer Tabelle der Hyastenus-Arten.

a. Cephalothorax oben glatt oder nur mit einzelnen Dornen (ohne Tuberkeln).

b. Nur je ein Dorn seitlich auf den Branchialgegenden, der bei einer Varietät (elongata OrTM). fehlt.

H. diacanthus (DE H.).

bb. Je ein Dorn auf den Branchialgegenden und zwei Dornen auf der Gastricalgegend.

bbb. Ausserdem noch ein Dorn am Hinterrande des Cephalothorax.

H. $\left.\operatorname{aries}(\text { Latr. })^{1}\right)$.

aa. Ceplalothorax oben uneben und höckerig (mit Dornen und Tuberkeln).

II. spinosus A. M.-E.2).

b. Cephalothorax wenig höckerig: Cardiacalgegend und obere Branchialgegend ohne Höcker und Dornen.

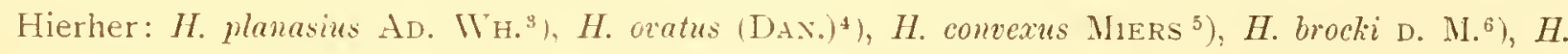
carribaeus RATHB. i\%.

bb. Branchialregion mit Dornen oder Tuberkeln. Cardiacal-und Intestinalregion höckerig oder dornig. c. Cardiacal- und Intestinalregion je nur von einem Höcker oder Dorn eingenommen (bisweilen vor dem Höclier der Cardiacalregion noch ein kleiner, nie aber neben ihm).

d. Gastrical- und Cardiacalregion in je einen stunspen Höcker erhoben.

dd. Gastricalregion in der Medianlinie mit zwei Höckern.

H. hilgendorfi D. M. ${ }^{\mathrm{s}}$.

e. Gastricalregion im Ganzen mit 6 Höckern, die in einer medianen Längsreihe (von 2) und in einer Querreihe angeordnet sind. Auf der Branchialregion mehrere dornförmige Höcker.

H. pleione (Нвsт.) ${ }^{4}$ ).

ee. Gastricalregion im Ganzen mit $\&$ Höckern, die in drei Längsreihen angeordnet sind. Auf der Branchialregion nur ein dornförmiger Höcker, die übrigen Höcker klein.

Hierher: H. japonicus Mrs. ${ }^{10}$ ), H. longipes (DAN. ${ }^{11}$ ).

ddd. Gastricalgegend in der Medianlinie mit drei Höcliern.

e. Gastricalgegend nur wit diesen drei Höckern. Branchialgegend nur mit zwei Dornen.

H. gracilirostris MRS. ${ }^{2}$ ).

ee. Gastricalgegend seitlich mit weiteren Höckern. Branchialgegend ebenfalls mit weiteren Höckern.

1) Vigl. Chorinus aries Milne-EDwards, H. N. Cr., I, I834, p. 315. - Ost-Indien.

2) A. Milne-Edwards, Nouv. Arch. Mus., S, 1872, p. 250. - Mozambique, Fidji.

3) ADA3s et White, Zool. loy. Samarang, 1S48, p. 9. pl. 2, fig. 4, 5. - China, Australien.

4) Lehana orata, DAxA. U. S. Expl. Exp. 1852 , p. 93 pl. 2, fig. I. Miers, Alert, 18S4, p. 522. - Sandwich-Ins., Amiranten.

5) Mlers, Alert, 1884, p. 196, pl. I8, B. - Australien.

6) DE MAN, Arch. f. Naturg., 53, I, I887, p. 22I, pl. 7, fig. 1. - Amboina.

7) Rathbun, Proc. U. S. Nation. Mus, 16, I893. p. 85, pl. 6, fig. 2. - Columbia: Sabanilla.

8) DE MAN, Joum. Linn. Soc., 22, 1888, p. 14, pl. 1, fig. 3. 4. - Mergui-Inseln.

9) Vgl. DE MAx, 1. c. 1887 , p. 225 , pl. 6, fig. 3, 1. c. I885, p. Is. - Mergui-Inseln, Amboina.

Io) Miers, Proc. Zool. Soc. London, I879, pl. I, fig. 2. - Japan.

11) Chorilia longipes DAxi, U. S. Expl. Exp., I852, p. 91, pl. 1, fig. 5. Strafsox, Boston Joum. Nat. Hist.. 6. 4, 1854, p. 455. Hyastenus longipes (DAx.) RathbuN, Proceed. U. S. Nation. Mus., I6, IS93, p. 85, pl. 7. - Von Alaska bis San Diego. Californien. Nach RathbiN soll $H$. japonicus hiermit identisch sein.

I 2) Miers, Ann. Mag. N. H. (5), IV, IS79, p. I2, pl. 4, fig. 7. - Fidji.

Jenaische Denkschriften VIII. 
f. Rostralhörner lang. Praeoculardorn und Dorn an der vorderen äusseren Ecke des. Basalgliedes der äusseren Antennen gut entwickelt.

H. elegans Mrs. ${ }^{3}$ )

ff. Rostralhörner kürzer. Praeoculardorn und Antennaldorn fehlend.

H. brevicornis nov. spec. cc. Cardiacalgegend mit einer Gruppe von Höckern, ebenso die Intestinalgegend mit mehreren Höckern. Höcker der Gastricalgegend sehr zahireich.

d. Rostralhörner an der Basis genähert.

dd. Rostralhörner an der Basis etwas entfernt von einander.

H. sebae WH. H. $\operatorname{oryx}$ A. M.-E. ${ }^{2}$ ).

\section{Hyastemus diaeanthus (DE HAAN).}

Vgl. Ortmann, Zool. Jahrb., I'II, 1893, p. 55. Ratheun, Proceed. U. S. Nat. Mus., 16, 1893, p. 85.

Die Rostralhörner divergiren etwas stärker als bei typischen Exemplaren.

Thursday Is land, 3 Ex. (SEMON coll.).

Verbreitung: Japan (de Haan, Stimpson, Miers, Ortmann, Rathbun); China (Stimpson, HelLER); Philippinen (Haswell, Mlers); Java (DE MaN); Singapur (Walker); Arafura-See (Miers); Australien (A. Milne-Fidwards, Haswell, Miers, Rathbli); Neu-Seeland (Haswell).

\section{Hyastenus brevicomis noc. spec. Tafel III, Fig. 3.}

Diese Art steht dem H. gracitirostris und dem H. elegans sehr nahe. Sie unterscheidet sich von ersterer durch zahlreichere Höcker auf dem Cephalothorax, von letzterer durch kürzere Rostralhörner, fehlenden Praeocular- und Antennaldorn und durch etwas andere Anordnung der Höcker der Branchialgegend.

In der Mittellinie der Gastricalgegend stehen drei kleine Höcker hinter einander, neben diesen jederseits drei kleinere Höcker, die zwei vorderen neben einander. Ferner steht je ein grösserer, dornähnlicher auf der Cardiacal- und Intestinalgegend, vor dem ersteren noch ein ganz kleiner. Auf den Branchialgegenden steht je ein grosser Epibranchialstachel, und zwischen diesem und dem Cardiacalhöcker steht noch ein kleiner Höcker. Mehr nach vorn liegen noch drei kleine Höcker, der vorderste hinter dem Postorbitalzahn, nach innen von den beiden hinteren findet sich ein undeutlicher Wulst. Unter dem Epibranchialdorn, nach den Pereiopoden zu liegt ein weiterer Höcher. Supraorbitalrand wie bei gracilirostris.

Die Pereiopoden stimmen mit gracilirostris überein, die Rostralhörner sind etwas kürzer.

$\mathrm{K}$ il wa, auf der Rhede, 8-IO m, I of I $q$ (ORTMANN coll.).

\section{Hyastemus sebae WHITE.}

White, Proceed. Zool. Soc. London, 15. 1847, p. 57. AnAms et White, Zool. Voy. Samarang. Crust., 1848, p. 11. DE MAN. Arch. f. Naturg., 53, 1, 1887, p. 223.

Mein Exemplar gehört nach der Bildung der Rostralhörner zu sebae (vergl. DE MAN und A. MiLNEEDWARDS, 1872, p. 252), die letzteren sind an der Basis genähert und divergiren etwas nach vorn. Sie sind aber kürzer als bei erwaclisenen ¿. Cephalothorax I5 mm lang, Rostralhörner 5,5 mm.

Thursday Island, I $q$ (SEMON coll.).

Terbreitung: Philippinen (AdAus et White); Amboina (DE MAN).

\section{Gattung: Naxia.}

Tabelle der Arten der Gattung Naxia:

a. In der Medianlinie des Cephalothorax auf der Gastricalgegend und Cardiacalgegend keine DornHöcker. Meren der Pereiopoden am distalen Ende oben ohne Dornen.

b. Accessorische Dornen der Rostralhörner nahe der Spitze (aufwärts gerichtet), fast so lang wie die etwas auswärts gerichteten Spitzen der Hörner selbst. Praeoculardorn stark entwickelt. Branchialgegend mit je einem Dorn.

N. sermulifera (GUÉR.).

bb. Accessorische Dornen der Rostralhörner kürzer. Praeoculardorn unentwickelt. Branchialgegend mit einem grösseren und $\mathbf{I - 2}$ kleineren Dornen.

N. hirta (A. M.-E.. $)^{3}$ ).

I) Mrers, Chall. Brach., ISS6, p. 58, pl. 6, fig. 3. - Neu-Guinea: Ki Isl.

2) A. Milne-Edwards, Nouv. Arch. Mus., 8, I872. p. 250, pl. I4, fig. I Mifers, Alert, i884, p. 395 und 522. DE MAN, Arch. f. Nat. IS87, p. 224, pl. 7, fig.2. - Westlicher Indischer Ocean: Providence Isl., Java. China-See, Philippinen, Australien. Neu-Caledonien.

3) Viaxioules hirta A. Milne-Edwards, Annal. Soc. entomol. France (4), V. IS65, p. I43, pl. 4, fig. I. Podopisa petersi, HilgenDorf, Mon. Ber. Ak. IV. Berlin. I8,8, p. 785, pl. I. fig. I -5. Naxia petersi MIERS. Alert. I854. p. 523. N. hirta MIERS, Chall. Bracls. 1586, p. 61. - Mozambique. Zanzibar, Amiranten. Philippinen. 
aa. In der Medianlinie des Cephalothorax eine Anzahl spitzer Dornhöcker auf Gastrical-, Cardiacalund Intestinalgegend. Meren der Pereiopoden am distalen Ende oben mit einem Dorı.

b. Praeoculardorn schrïg nach vorn und oben gerichtet.

c. Rostralhörner kürzer als der halbe Cephalothorax. Accessorischer Dorn nahe der Spitze. Vier kräftige Dornen in der Medianlinie des Cephalothorax. Drei kräftige Dornen auf den Branchialgegenden.

N. hystrix MRs. ${ }^{1}$ ).

cc. Rostralhörner länger àls der halbe Cephalothorax. Accessorischer Dorn entfernter von der Spitze. In der Mittellinie des Cephalothorax und auf den Branchialgegenden treten noch weitere Dornen hinzu.

d. Rostralhörner stärker divergirend. Die Dornen des Cephalothorax schwächer und weniger zahlreich.

N. mammillata ORTH. ${ }^{8}$ ).

dd. Rostralhörner weniger divergirend. Dornen des Cephalothorax sehr stark entwickelt und zahlreich.

N. robillardi $\mathrm{MRS}^{3}{ }^{3}$.

bb. Praeoculardorn aufwärts gerichtet und gegen die Spitze schwach rückwärts gebogen, sehr kräftig. In der Mittellinie des Cephalothorax vier, auf den Branchialgegenden drei Dornen. Rostralhörner lang.

N. cerastes nov. spec.

\section{Naxia serpuliferr (GUÉRIN).}

Mrlie-Emwakds, H. N. Cr., I, 1834, p. 313. Haswell. Catal. Austral. Crust., 1882, p. 21. Miers, Rep. Zool. Coll. Alert, 1884, p. 196.

Der Höcker der Intestinalregion ist bei jungen Exemplaren kräftiger, fast dornförmig. Bei dem grössten mir vorliegenden Exemplar $(q)$ ist er stumpf und gerundet. Hinter ihm liegt bei allen Exemplaren dicht am hinteren Rande des Cephalothorax ein kleinerer Höcker.

Thursday Island, 9 Ex. (SEyon coll.).

Verbreitung: Australien (Muxe-Enwarns): Raffles Bay (Miers), Port Essington (Haswell), Thursday lsland (Haswell, Mers), Shark Bay (Miers).

\section{Naxia cerrstes nov. spec. Tafel 111, Fig. 4.}

Rostralhörner über halb so lang als der Cephalothorax (Rostralhörner: I2 mm, Cephalothorax: I9 $\mathrm{mm}$ ), stark divergirend. Accessorischer Dorn etwas entfernt von der Spitze. In der Mittellinie des Cephalothorax stehen vier kräftige Dornen, davon zwei auf der Gastricalgegend, je einer auf der Cardiacalund Intestinalgegend. Auf der Gastricalgegend steht neben dem vorderen Dorn jederseits ein $k l e i n e r e r$, ausserdem noch je drei kleine Höcker. Branchialgegend mit drei spitzen Dornen und einem Höcker neben dem Cardiacaldorn. Pterygostomialgegend mit zwei über einander stehenden Dornen, der obere in der Höhe des Auges. Vordere Ecke des Mundfeldes mit einem Dorn. Von diesem ziehen sich fünf Höcker über der Basis der Pereiopoden nach der Branchialgegend hin. Praeoculardorn aufwärts gerichtet und leicht rückwärts gekrümmt, etwa so lang wie die vorderen Dornen der Gastricalgegend. Festes Stielglied der äusseren Antennen an der vorderen äusseren Ecke mit einem spitzen Dorn, ein weiterer, nach aussen und abwärts gerichteter Dorn stelut nahe der Basis desselben.

Meren der Pereiopoden am distalen Ende oben mit einem schlanken Dorn.

Die Bedornung des Cephalothorax erinnert einigermaassen an N. hystrix. Die eigenthümliche Entwickelung des Praeoculardornes unterscheidet aber die vorliegende Art von allen bereits bekannten.

Thursday Island, I ठ (SEMON coll.).

\section{Schizophrys aspera (MLNE-EDWARDS).}

Vgl. Ontmann, 1. c. 1893, p. 5ī. Rathbux, Proceed. U. S. Nat. Mus., 16, 1893, p. 91.

Amboina, 3 juv. (Senox coll.).

Verbreitung: Durch das ganze Indo-Pacifische Gebiet, rom Rothen Meer und Madagascar bis Japan, Australien und Samoa.

Tgl Ortuaxi, l, c. p. 59

Vicippe philyra (HERBST).

Meine Exemplare stimmen völlig mit dem von Rıchters (Beitr. Meeresf. Mauritius und Seychellen, I880, p. I42, pl. I5, fig. I-5) beschriebenen o der Micippe philyra latifions überein.

1) Miers, Chall. Brach., I\$86, p. 60, pl. 6, fig. 4. - Amboina.

2) Ortuaxw, Zool. Jahrb., VII, 1893, p. 56, pl. 3, fig. 7. - Japan.

3) Miers, Proceed. Zool. Sc., 1852, p. 33 h pl. 20, fig. 1. - Mauritius. 
Dar-es-Sal a a m, Upanga-Riff, 3 (ORTMAnN coll.).

Verbreitung: Rothes Meer bis Tongatabu. Von der ost-afrikanischen Küste bisher noch nicht bekannt; die nächsten Fundorte sind: Rothes Meer (RüPPELl, Heller, A. Milne-Edwards); Cap (?) (A. Milne-Edwards); Madagascar: Nossi Bé (Lenz und Rıchters); Mauritius (Milne-Edwards, Richters).

\section{Miciple thalia (HERBSt) var.}

Vgl. Ortuani, 1. c. 1893 , p. 60 .

Das vorliegende Exemplar würde in den sonstigen Charakteren mit der Micippe thalia caledonica bei Kossmann (Erg. Reis. Roth. Meer, I, I877, p. 8) übereinstimmen: jedoch ist das Rostrum ganz eigenthümlich gebildet. Dasselbe ist zunächst senkrecht nach unten geneigt und biegt dann wieder, etwa von der Mitte ab, schräg nach rückwärts und unten, d. h. gegen die Unterfläche des Körpers zu. Es verschmälert sich gegen die Spitze, die kurz zweilappig ist, der Rand besitzt keine Dornen. Die Enddornen sind also nicht, ivie sonst bei thalia, divergirend oder fast parallel, sondern convergiren offenbar, indem sie dabei auf eine längere Strecke median verwachsen bleiben. Bei den vielfachen Variationen, die die Arten der Gattung Micippe zeigen, scheue ich mich, diese Form als besondere Art einzuführen.

Thursday Island, I $q$ (SEmon coll.).

Verbreitung der MI. thalia: Vom Rothen Meer und Natal bis Japan und Neu-Caledonien.

Familie: Periecridae.

Tinrinia gracilis DANA.

Vgl. Ortmann, 1. c. p. 62 .

Amboina, I $\delta$ (SEMON coll.).

Verbreitıng: Singapur (Ortmann); Sulu-See (Dana); Neu-Guinea (Ortmann).

Tylocareinus styx (HERBST).

Vgl. Ortmani, l. c. p. 62 .

Amboina, I $q$ (SEuon coll.).

Dar-es-Sal a am, Upanga-Riff I $\delta$ (OrTmann coll.).

Verbreitung: Vom Rothen Meer bis zu den Fidji-Inseln. - Neu für Ost-Afrika, die nächsten Fundorte sind: Rothes Meer (A. Milne-Edwards, de Man); Mauritius (Milne-Edwards, Adans et White). - Von Amboina von DE MAN erwähnt.

\section{Familie: Platyonychidae.}

Hortumuns pulchcllus (MACLEAY).

Xaiva pulchella Macl. Krauss, Südafrik. Crust., 1843, p. 27.

Platyonychus pulch. (Mach.) A. Hilne-Ebwards, Arch. Mus., X, 1861, p. 412.

Cap: Port El isabeth, 2 I $q$ (Mus. Strassburg).

Verbreitung: Süd-Afrika (Macleay, A. Milne-Edwards).

Platyonychus bipustulatus MrLnE-EDwards.

Vgl. Ortmann, Zool. Jahrb., VII, 1899, p. 65.

Cap: Port Elisabeth, 3 (Mus. Strassburg).

Verbreitung: Chile, Patagonien, Neu-Seeland, Süd-Australien, Cap. - Ausserdem Japan (DE HaAn, Ortuane) und Indien (A. Milne-Edivards).

\section{Familie: Carupidae.}

Carupa lacviuscula HELLER.

Vgl. Ortmann, 1. c. p. 68 .

Amboina, I $q$ juv. (SEMon coll.).

Verbreitung: Rothes Meer (DE Man); Japan (Ortuann); Amboina (DE Man); Timor (DE Man);

Samoa (Ortmann); Tahiti (Heller). 
Familie: Portunidae.

Neptume (Aehclons) gramulatus (Milne-Edwards).

Tgl. Ortmanx, 1. c. p. i2.

Dar-es-Sala am, im Hafen, I o (OrTMAns coll.).

Verbreitung: Vom Rothen Meer bis Japan und Tahiti. - Von der ost-afrikanischen Küste noch nicht erwälnt; die nächsten Fundorte sind: Rothes Meer (A. Milne-Edwards); Madagascar: Nossi Bé (Lenz und Richters); Mascarenen (Milne-Edwards, Hoffmann, Richters, Ortmann); Seychellen (Miers).

Noptumus pelagiens (LINNÉ).

Vgl. OrTuax, 1. c. p. T4.

Auch die vorliegenden beiden jugendlichen Exemplare von der Thursday-Insel entsprechen vielleicht dein N. armatus A. MiLNE-EDWARDs.

Amboina, 3 Ex., Thursday Island, 2 juv. (Semon coll.).

Verbreitung: Ueberall im Indo-Pacifischen Gebiet.

Vgl. Ortuass, 1. c. p. 75 .

\section{Neptumus somgnimolemtus (HERBst).}

A mboina, 2 juv. (SMON coll.).

Dar-es-Salaam, im Hafen, I o 2 juv. (Ortmann coll.).

Verbreitung: Indischer Ocean bis Japan, Australien und zu den Sandwich-Inseln. - Wird vom Cap (Heller) und Réunion (Hoffunan) angegeben, von Amboina noch nicht bekannt.

\section{Scylla semerta (FORSKAL).}

Tgl. Orthasi, 1. c. p. To.

Dar-es-Salaam, im Hafen, I d (ORTMAN coll.).

Verbreitung: Rothes Meer bis Natal, Japan, Australien und Tahiti.

\section{Familie: Thalamitidae.}

\section{Geniosoma sexdentatum (HERBST).}

Vgl. A. Milne-Edwards, Arch. Mns., X, 1861, p. 372. De Max, Not. Leyd. Mus., I, 1879, p. 59.

Thursday Island, I juv. (SEMon coll.).

Verbreitung: Rothes Meer (Röppell); Mayotte (A. Milne-Edwards); Bombay (A. MilneEdwards); Sumatra (A. Milne-Edwards); Amboina (de Man).

\section{Gomiosoma affine (DANA).}

Charybdis affunis DAxA. U. S. Expl. Exp., 1852, p. 286, pl. 17, fig. 12.

Goniosoma affue (Dex.) A. MLxe-EdWAlus, Arch. Hus., X, 1861, p. 384. we Max, Journ. Linn. Soc. Zool., 22, 1888, p. 80 , pl. 5, fig 2 .

Dar-es-Sala am, im Hafen, i q (ORThANn coll.).

Verbreitung: Singapur (DANA); Mergui-Inseln (DE MAN).

Goniosoma (Thalamomyx) danae A. Milne-EDwards.

Vgl. Ortmaxi, 1. c. 1893 , p. 83 .

Meine Exemplare unterscheiden sich von typischen dadurch, dass der vierte Anterolateralzahn etwas kleiner ist als der folgende. Die Dornen der Scheere sind sehr variabel, meist sind drei gut entwickelt, häufig aber auch der obere dicht vor dem beweglichen Finger gelegene. Granulirung des Cephalothorax ebenfalls variabel.

Diese Form ähnelt ausserordentlich jungen Exemplaren von Thalamita sima. Letztere, die mir in gleicher Grösse vorliegen (vgl. unten), unterscheiden sich nur durch deutlichen Dorn über der Basis des beweglichen Fingers und durch stärker gekielte Hände. Vielleicht ist Thalamonyx überhaupt nur als Jugendform von Thalamita aufzufassen.

K ilwa, Rhede, 8-Io m, 9 J 4 f; Dar-es-Sala am, im Hafen, I I 7 (ORTMAN coll.). ORTMANN).

Verbreitung: Neu-Caledonien (A. Milne-Edwards); Samoa-Inseln (A. Milne-Edwards, 


\section{Thalamita admete (HERBST).}

Vgl. Ortmans, 1. c. p. 83 .

Amboina, I $\delta$ (SEMon coll.).

Verbreitung: Vom Rothen Meer bis Natal und Tahiti. - Von Amboina noch nicht erwähnt.

Thalamita savignyi A. MILNE-EDWARDS.

A. Mrlne-Edwards, Arch. Mus. Paris, X, 1861, p. 357. A. Milne-Enwards, Nouv. Arch. Mus., IX, 1873, p. 163. De Man, Not. Leyd. Mus., II, 1880 , p. 180 ve MaN, ibid., III, 1881, p. 99. Miers, Alert, 1884, p. 230. DE MaN, Journ. Linn. Soc. Zool., 22, 1888, p. 73.

Hände oben zwischen den Kielen mit zahlreichen Körnern. Innenseite, wie auch der untere Theil der Aussenseite glatt und hierdurch von der typischen savignyi abweichend und sich der admete nähernd. Bei letzterer fehlen jedoch die Granulationen auch auf dem oberen Theil der Aussenseite.

Dar-es-Sala am, im Hafen, I J (OrTMANn coll.).

Verbreitung: Rothes Meer (A. Milne-Edwards): Golf von Suez (Miers), Djiddah (DE Man); Mergui-Inseln (DE MAN); Nordwest-Australien (Miers); Neu-Caledonien (A. Milne-Edwards).

Thalamita sima MiLne-EDwards.

Vgl. Ortmane, 1. c. p. 84 .

Thursday Island, I \& 8 juv. (SEMON coll.).

Verbreitung: Rothes Meer, Ost-Afrika bis Japan und Australien.

\section{Thalamita intermedia MiERS.}

Miers, Chall. Brach., 1886, p. 196, pl. 16, fig. 1.

Thursday Island, I $\delta$ (SEMON coll.).

Verbreitung: Von dieser Art wurde bisher nur ein einziges Exemplar (ठ) bekannt, das vom Challenger in der Torres-Strasse in 8 Fad. Tiefe gedredgt wurde.

Vgl. Ortmann, l. c. p. 84 .

Thalamita mymma (HERBST).

Amboina, I o (Semon coll.).

Verbreitung: Vom Rothen Meer und Natal bis Japan und Tongatabu.

Thalamita stimpsoni A. MILNE-EDWARDS.

Vgl. Ortmann, l. c. p. 85 .

Thursday Island, I ¿ (SEmon coll.).

Verbreitung: Von Ceylon bis zu den Samoa-Inseln.

\section{Thalamita crenata (LATREILLE).}

Vgl. Ortmann, 1. c. p. 86 .

Thursday Island, I $\delta$ I $q$ (SEMON coll.).

Kilwa, an Strande, I $\delta$; Dar-es-Sala a m, im Hafen, zahlreiche Ex. (OrTMANn coll.).

Verbreitung: Vom Rothen Meer bis Natal, zu den Liu-Kiu- und Fidji-Inseln.

\section{Familie: Parthenopidae.}

\section{Lambrus pelagicus RÜPPELL.}

Vgl. Ortmann, Zool. Jahrb., VII, 1893, p. 414.

K ilwa, Rhede, 8-IO m, I $\delta$ (ORTMANn coll.).

Verbreitung: Vom Rothen Meer und Zanzibar bis zu den Samoa-Inseln.

Tabelle der Arten der Untergattung: Aulacolambrus.

a. Meren der vier hinteren Beinpaare dornig.

L. whitei A. M.-E.').

aa. Meren der vier hinteren Beinpaare nicht dornig.

b. Cephalothorax hinter den Augen etwas eingeschnürt. Aussenrand der Scheere mit 5 grösseren Dornen.

1) L. carinatus AdAms et White, Zool. Voy. Samarang. Crust., 1848, p. 27, pl. 5, fig. 3. L. whitez A. Milne-EdWardS, Nouv. Arch. Mus. Paris, S, 1872 , p. 260, Anmerk. - Borneo, China. 
c. Zwischen den 5 grossen Dornen des Aussenrandes der Scheere stehen keine weiteren Dornen oder Höcker. Hinterrand des Cephalothorax jederseits mit drei Dornen (der grosse Seitendorn eingerechnet).

L. diacanthus D. H.').

cc. Zwischen den 5 grossen Dornen stehen noch kleinere Dornen oder Höcker. Hinterrand des Cephalothorax jederseits mit zwei Dornen.

d. Dornen des Aussenrandes der Hand und des Hinterrandes des Merus der Scheerenfüsse kräftiger als die übrigen Dornen der Scheerenfüsse. Körper und Scheeren weniger dicht granulirt, Cephalothorax im Verhältniss breiter.

L. pisoides $\mathrm{AD}$. WH.

dd. Dornen der Handkanten und des Vorder- und Hinterrandes des Merus nicht auffällicr verschieden. Körper und Scheeren dichter granulirt. Cephalothorax im Verhältniss länger.

L. sculptus A. M.-E. ${ }^{2}$ ),

bb. Cephalothorax hinter den Augen nicht eingeschnürt.

c. Oberfläche des Cephalothorax mit einer Anzahl grosser, scharfrandiger, oben abgeflachter Warzen besetzt. Aussenrand der Palma mit 6 grossen Dornen. Vordere Unterfläche des Merus und Unterfläche der Palma mit grossen, gerundeten Warzen.

L. lecanora n. sp.

cc. Oberfläche des Cephalothorax nicht mit solchen grossen WVarzen, sondern ungleichmässig granulirt. Aussenrand der Palma mit 6-8 grossen Domen. Unterfläche des Merus und der Palma nicht warzig, sondern granulirt oder glatt.

L. hoplonotus AD. $\mathrm{WVH}_{\text {. }}$

Lambrus (Aulacolambmus) pisoides AdAns et IVHite.

Lambrus pisoides ADAns et Mn1TE, Zool. Voy. Samarang. Crust., 184s, 1. 28, pl. 5, fig. 4.

L. (Aul.) diacanthus OrTmaxi, l. c. p. 415 (non de Hadi).

Mein Exemplar stimmt mit der Beschreibung und Abbildung in allen Einzelheiten überein. Das von mir 1. c. als L. diacanthus angeführte Exemplar gehört ebenfalls hierher. Vielleicht aber sind die drei Arten: diacanthus, pisoilles und sculptus zu vereinigen.

Dar-es-Sala am, im Hafen, 5 m, I d (ORTMANn coll.).

Verbreitung: Philippinen (ADAms et White); Japan, Kadsiyama (OrTaAn).

\section{Lambrus (Aulacolambrus) lecamoru nov. spec. Tafel Ill, Fig. 5.}

Cephalothorax im Umriss dreiseitig (Länge $23 \mathrm{~mm}$, Breite ca. $32 \mathrm{~mm})^{3}$ ), hinter den Augen nicht eingeschnürt. Rostrum spatelförmig, mit stumpfer Spitze, wenig vorspringend, schwach abwärts geneigt, seine obere Fläche glatt. Oberfläche des Cephalothorax mit groben Warzen besetzt, die auf der Cardiacalund den Branchialgegenden gerundet sind. Die Warzen der Gastricalgegend und oberen Hepaticalgegend sind oben abgeflacht und haben scharfe Ränder. Eine zusammenfliessende Gruppe derartiger Warzen steht dicht hinter jeder Orbita. Auch der Unterrand der Orbita ist mit kleinen Gruppen solcher abgeflachten Warzen besetzt. Zwischen den grösseren Warzen stehen kleinere und kleinste. Vorderseitenrand mit einer Reile ron sechs Zähnen, deren vorderste warzenförmig sind, während die hinteren allmählich kurz dorn. förmig werden. Seitendorn stark, schräg nach hinten und aussen gerichtet, seine obere Fläche mit Körnern besetzt. Am Hinterrand des Cephalothorax steht neben dem Seitendorn noch je ein kleinerer Dorn, sonst finden sich dort nur gröbere und feinere Körner. Pterygostomialrinne sehr deutlich.

Merus der linken ersten Pereiopoden am Hinterrande mit 5 Dornen (ein kleinerer sechster steht nocl am proximalen Ende, auf diesen folgt noch eine Warze). Oberer Vorderrand des Merus mit sieben Körnern, die abwechselnd grösser und kleiner sind. Unterer Vorderrand stumpf, wie die vordere UnterHäche mit einer Anzahl grosser, gerundeter Warzen besetzt. Carpus am Aussenrand mit fünf Dornen, der vorletzte am distalen Ende der grösste, Oberseite mit einigen Höckern, untere Kante mit einer Reihe kleiner Warzen. Aussenkante der Hand mit sechs grossen, lang-dreieckigen, abgeflachten Dornen (der vorderste ist abgebrochen), zwischen diesen stehen kleine Höcker, von denen der zwischen dem 3. und 4. Dorn kurz dornförmig wird. Obere Innenkante der Hand mit acht Dornen, die lileiner sind als die des Aussenrandes und von der Basis zur Spitze etwas an Grösse zunehmen. Auf der oberen Fläche der Hand stehen vier grössere und einige kleine Tuberkeln, der vorderste derselben (über der Basis des beweglichen Fingers) zeigt eine ähnlich abgeflachte Fläche wie die vorderen IVarzen des Cephalothorax. Unterkante der Hand mit einer Reihe grober, gerundeter WVarzen, und ähnliche Warzen finden sich auf der vorderen und hinteren. Unterfläche. Beweglicher Scheerenfinger an der Basis mit drei Dornen, einer auf der Aussenkante, zwei auf der Innenkante. Spitzen beider Finger schwarz.

1) DE HAAN. Faun. japon. Crust. dec., 4, 1839, p. 92, pl. 23, fig. 1. - Japan.

2) A. Milne-Edwards, Nouv. Arch. 8, i872, p. 258, pl. I4. fig. 3. - Neu-Caledonien.

3) Da der Seitendorn der rechten Seite abgebrochen ist, Iässt sich die Breite nicht genau messen. 
Uebrige Pereiopoden mit ungezähntem, glattem Merus. Krallen ziemlich gerade.

Dem vorliegenden Exemplar fehlt der rechte Scheerenfuss und auch die rechte Branchialgegend ist verletzt, da der grosse Seitenstachel abgebrochen ist.

Thursday Island, i o (Senon coll.).

\section{Lambrus (Aulucolambrus) hoplonotus ADAMS et VWHTE.}

Adans et White, Zool. Toy. Samarang Crust., 1848, p. 35, pl. 7, fig. 3. A. Milxe-Edwards, Nouv. Areh. Mus., 8, 1872, p. 258. Miers, Ann. Mag. N. H. (5), IV, 1879, p. 22 u. 24, pl. 6, fig. 6, 7. Haswell, Catalog. Austral. Crust., 1882, p. 33. Miers, Rep. Collect. Alert, 1884, p. 201. Mieks, Chall. Brach., 1886, p. 98, pl. 10 , fig. 5.

Miers hat eine Reihe von Formen dieser Art beschrieben, denen ich eine weitere hinzufügen muss:

var. tentifrons nov. Tafel III, Fig. 6.

Die Körner der Oberfläche des Cepholothorax stimmen mit der var. granulata Miers (vgl. Chall. pl. 10, fig. 5) völlig überein, und auch sonst hat mein Exemplar mit dieser Varietät grosse Aehnlichkeit. Das Rostrum ist jedoch an der Spitze fünfzähnig, der mittlere Zahn der längste, die seitlichen kornförmig. Auch der obere und untere Rand der Orbita zeigt Körner, von denen besonders zwei nahe der äusseren Ecke fast dornförmig werden. Der Vorderrand des Merus der Scheerenfüsse trägt nicht gerundete Körner, sondern spitze, ungleiche Zähnchen, von denen $3-4$ grösser sind. Der Aussenrand der Hand hat acht spitze, lange Dornen, von denen der 2. und 4. kürzer sind. Zwischen diesen Dornen steht je ein spitzer Höcker. Die Basis des beweglichen Fingers trägt oben drei Dornen, einen auf der äusseren, zwei auf der inneren Kante.

A mboina, I \& (SEMON coll.). gefunden :

Verbreitung: Die verschiedenen Formen des L. hoplonotus wurden an folgenden Localitäten

hoplonotus typicus: Neu-Caledonien (A. Milne-Edwards); Cap York (Miers); Torres-Strasse; Darnley Isl. (Haswell); Cap Grenville (Haswell); Albany-Passage (Haswell); Port Molle (HASWELL).

var. gramulosa Miers: Philippinen (Miers); Torres-Strasse (Miers); Queensland (Miers).

var. longioculis Miers: Australien: Percy Islands, $2 \mathrm{I}^{0} 50^{\circ} \mathrm{S}$., $150^{\circ} 20^{\circ}$ O. (Miers); Neu-Guinea (Miers). var. planifrons Miers: Ceylon (Miers).

var. curvispinus Miers: Java-See (MiERs).

\section{Cryptopodia spatulifions MIERS.}

Mifrs, Aun. Mag. N. H. 5), IV, 1879, p. 26, pl. 5, fig. 10. Haswell, Catal. Austral. Crust., 1882, p. 37. Mitrs, Rep. Zool. Coll. Alert, 188t, p. 203.

Thursday Island, I $\delta$ I $q$ (SEMon coll.).

Verbreitung: Australien: Port Jackson (HAswell), Thursday Island und Prince of Wales Channel (Miers), Shark Bay (Miers); Borneo (Miers, var. laevimana).

\section{Familie: Eumedonidae.}

\section{Gonctomotus pentagonus WHITE.}

White, Proceed. Zool. Londou, 15, 184 $\overline{7}$ p. 58. Adans et White, Zool. Voy. Samaraug Crust., 1848, p. 33, pl. 6, fig. 7. Miers, Proceed. Zool. Soc. London, 1879, p. 29. Haswell. Catal. Austral. Crust., 1882, p. 38. Miers, Alert, 1884, p. 204.

Thursday Island, I $\delta$ (SEMON coll.).

Verbreitung: Borneo (AdAns et White); Java-See: Billiton Isl. (Miers); Thursday Isl. (MIERs); Nordostküste Australiens (Miers): Port Denison (HASWELL).

Familie: Jenippidae.

\section{Myomenippe panope (HERBST).}

* Cancer harlwoichi Grar, Zool. Misc., 1831, p. 40.

Menippe granulosa Strahi, Arch. f. Naturg., 27, 1861, p. 105. A. Mrlaxe-Edwards, Annal. Soc. entom. France (4), VII, 1867 , p. 275.

Menippe panope (Нват.) v. Martens, Arch. f. Naturg., 38, 1872, p. 87.

Myomenippe duplicidens Hugexdorf, Mon. Ber. Akad. Wiss. Berlin, 1878, p. 796, Anmerk.

Menippe (Myomenippe) panope (Нвsт.) Miers, Aunal. Mag. N. H. (5), V, 1880, p. 232. 
Dar-es-Salaam, im Hafen, I $q$; Upanga-Riff, I of (ORTMAnn coll.).

boina (MIERS).

Verbreitung: Java (Miers): Batavia (A. Milne-Edwards); Celebes (Hirgendorf); Am-

\section{Pseudozius caystrus (ADAMS et WhITE).}

Tgl. Ortuax, Zool. Jahrb., VII, 1893, p. 434.

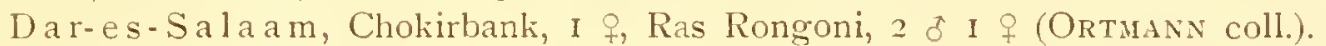

Verbreitung: Mauritius (Rrchters); Bonin-Inseln (Stimpson); Wake-Insel (DANA); SamoaInseln (OrTuAN); Paumotu-Inseln (DANA).

\section{Eurycarcims matalensis (KRAUSS).}

Galene natalensis Erauss, Südafrik. Crust., 1843, p. 31, pl. 1, fig. 4. Hofraxis, Crust. Echinod. Madagascar, 1874, p. 4. Kussuaxi, Zool. Ergebn. Reis. Roth. Meer, 1, 1877, p. 37.

Eurycarcinus grandidieri A. Milne-Eirwards, Annal. Soc. entomol. France (4), Tit, 1867, p. 27t. A. Milxe-Edrards Nouv. Arch. Mus. Paris, IV, 1868, p. 80, pl. 19, fig. 13-113.

Eurycarcinus natalensis (Ku.) Hibgexdorf, Mon. Ber. Akad. Wiss. Berlin, 1878, p. 792. Lexz u. Richrers, Beitr. Crustaceenfaun. Madagascar, 1881, p. 2.

Dar-es-Sala a m, im Hafen bei Mtoni, I f (ORTMAn coll.).

Verbreitung: Natal (Krauss); Inhambane (Hilgendorf); Ibo (Hilgendorf); Nossi Faly (HoffMans); Nossi Bé (Lenz u. Rıchters); Zanzibar (A. Milne-Edwards); Rothes Meer (Kossuans).

\section{Pilumulus vespertilio (FABRICIUS).}

Tgl. Orruaxi, 1. c. p. 438.

Die Exemplare vom Upanga-Riff sind bräunlich gefärbt, die längeren Haare gelblich, die von der Chokirbank sind mit grauen Haaren besetzt. Vielleicht ist dies eine Anpassung an den Aufenthaltsort, da auf dem Upanga-Riff die braunen Farbtöne des lebenden Riffes vorherrschen, während auf der Chokirbank hellere Farben auftreten, bedingt durch Korallendetritus und Sandflächen.

D a r-es-Sa l a a m, Chokirbank, $2 \delta 2$, Upanga-Riff, I 83 (ORTMann coll.).

Verbreitung: Rothes Meer bis Mozambique, zu den Liu-Kiu-Inseln, Australien und Samoa.

Pilumnus infrocilirris nox. spec. Tafel III, Fig. 7 .

Cephalothorax dicht mit kurzen, fast filzigen Haaren besetzt. Stirnrand zweilappig, vom oberen Augenhöhlenrand nicht durch eine Kerbe getrennt. Aeussere Orbitalecke von einem kleinen, stumpfen Höcker gebildet. Unterer Orbitalrand aus einem dicken, etwas zweilappigen Wulst bestehend. Vorderseitenrand mit drei kräftigen Zähnen, die nicht abgeflacht sind, sondern stumpflich-kegelförmig. Hinter dem letzten steht noch ein ganz kleiner, undeutlicher, accessorischer Höcker. Auf der Pterygostomialgegend stehen zwei stumpfe, aber deutliche Höcker, der eine unter dem Zwischenraum zwischen der äusseren Orbitalecke und dem ersten Seitenrandzahn, der andere unter der Lücke zwischen dem ersten und zweiten Seitenrandzalın. Oberfäche des Cephalothorax gegen den Vorderseitenrand zu hinter dem ersten Seitenrandzahn mit einem Höcker und ferner ebenda und gegen den Supraorbitalrand und Stirnrand mit einigen wenigen Körnern.

Carpus der Scheerenfüsse mit grossen, gerundeten Warzen besetzt, deren Oberfläche fein granulirt und unbehaart ist, die Zwischenräume zwischen den Warzen sind filzig. Hände auf der oberen Aussenfläche mit ebensolchen Warzen, die nach unten kleiner werden und unter dem Filz verschwinden. Bei der grossen Hand ist die untere Hälfte der Aussenfläche naclit und glatt. Die Scheerenfinger sind schwarz, an der kleinen Hand stark, an der grossen schwach gefurcht. Basis des beweglichen Fingers oben granulirt und behaart.

Hintere Beine dicht behaart. Carpus und Propodus oben mit ähnlichen Warzen wie die Scheerenfüsse.

Diese Art steht dem Pil. calculosus Dand (U. S. Expl. Exp., 18j2, p. 238, pl. 13, fig. 12) von Madeira (?) sehr nahe, unterscheidet sich aber vornehmlich: I. durch den unteren Orbitalrand, der bei calculosus dreizähnig ist, 2. durch die rundlichen, nicht länglichen Warzen der Beine und Scheeren, 3. durch etwas ungleiche Scheeren.

Cap: Port Elisabeth, $3 \delta 2 q$ (Mus. Strassburg).

Familie: Kanthidae.

Cymo andreossyi (SAvigny).

Vgl. Orminas, Zool. Jahrb., VII, 1893, p. 443.

Jenaische Denkschriften VIII. 
Dar-es-Sal a a m, Chokirbank, I o (OrTMAnN coll.).

Verbreitung: Rothes Meer bis Tahiti. - Von Ost-Afrika bisher noch nicht erwähnt, die nächsten Fundorte sind: Rothes Meer (Heller); Mergui-Inseln (DE MAN).

Tantho exaratus (MILNE-EDwARDs).

var. typica ORTMANN.

Vgl. Ortmann, 1. c. p. 445 .

Kilwa, am Strande, I I I $q$, Dar-es-Salaam, Ras Rongoni, 2 (ORTMann coll.).

Verbreitung: Vom Rothen Meer an der Ostküste Afrikas bis Natal, ferner bis Neu-Caledonien und zu den Sandwich-Inseln.

\section{Actrea tomentosa (MILnE-EDivards).}

Tgl. Ortulani, 1. c. p. 453.

Dar-es-Sala a m, Upanga-Riff und Chokirbank, 5 \& 5 क (Ortuann coll.).

Verbreitung: Rothes Meer bis Mozambique und Tahiti.

Actaea rufopunctata (MiLne-Edwards).

Vgl. Ortmann, 1. c. p. 454.

D a r-es-S a la a m, Upanga-Riff, I $q$, Chokirbank, I $q$ (ORTMANN coll.).

Verbreitung: Rothes Meer, Indischer Ocean, Pacifischer Ocean, nördlich bis Japan. Ferner im Atlantic auf der amerikanischen und afrikanischen Seite. - Wurde bisher von Ost-Afrika noch nicht erwähnt, dagegen von den Mascarenen (Milne-Edwards, HoffMans) und Seychellen (Miers).

\section{Actrea cavipes (DANA).}

Vgl. OrtaAns, l. c. p. 456.

Die vorliegenden Exemplare stimmen mit der von mir 1. c. erwälınten-japanischen Form überein, d. h. die Oberfläche des Cephalothorax ist zwischen den Körnern etwas klein-grubig und der Carpus der Scheerenfüsse zeigt eine besonders grosse, gerundete Grube.

Dar-es-Sal a am, Chokirbank, an lebenden Korallen, I of i q (ORTMann coll.).

Verbreitung: Neu-Caledonien (A. Milne-Edwards); Fidji-Inseln (Dana); Samoa-Inseln (Dana, Ortmann); Liu-Kiu-Inseln (Ortmann); Süd-Japan: Kagoshima (Ortuann). - Aus dem Indischen Ocean bisher noch unbekannt.

\section{Lophozozymus lodome (HERBST).}

Vgl. Ortmana, 1. c. p. 457.

Das vorliegende Exemplar ist typisch.

Cap: Port Elisabeth, I $q$ (Mus. Strassburg).

Verbreitung: Ibo (Hilgendorf); Mozambique (Miers); Mauritius (Milne-Edvards, Adas et White); Amboina (DE MAN); Neu-Caledonien (A. Milne-Edwards); Tahiti (Heller); Fidji-Inseln (ORTMANN).

\section{Zoะymus ceneus (LINNË).}

Vgl. Ortuani, 1. c. p. 458 .

Da r-es-Sal a a m, Upanga-Riff, unter lebenden Korallblöcken, 4 I I $q$ (OrTMAnN coll.).

Verbreitung: Rothes Neer bis zu den Paumotu-Inseln. - Von Ost-Afrika bisher noch nicht erwähnt, die nächsten Fundorte sind: Rothes Meer (Heller, Miers); Mascarenen (Hoffmann, Richters).

\section{Lophactael cristata A. MiLnE-EDwards.}

A. Minne-Edwards, Nouv. Arch. Mus. Paris, I, 1865, p. 246, pl. 16, fig. 1. de Man, Not. Leyd. Mus., III, 1881, p. 95. DE MaN, Arch. f. Naturg., 53, 1, 1887, p. 246.

Kiel der Hand granulirt, wie es A. Milne-EDwards angiebt, und mit dessen Beschreibung meine Exemplare völlig übereinstimmen. Exemplare von L. granulosa, die mir von Neu-Caledonien und den Samoa-Inseln vorliegen, zeigen keine Spur eines Kieles.

Dar-es-Sal a m, Chokirbank, 2 (ORTMann coll.).

Verbreitung: Rothes Meer: Djiddah (DE MAN); Zanzibar (A. Milne-Edwards); Java: Insel

Edam und Noordwachter (DE MAN); Cochinchina (A. Milne-Edwards). 
Tgl. Ortuax, l. c. p. 460.

Amboina, $3 \delta$ I $q$, Thursday Island, I $\delta$ (SEMox coll.).

Verbreitung: Vom Rothen Meer und den Mascarenen bis Japan und zu den Paumotu-Inseln.

Plymolins muglatus (MILNE-EDwards).

Vgl. Ortuax, 1. c. p. 454 .

Amboina, I juv. (SEMON coll.).

Dar-es-Salaam, I $\delta+q$ I juv. (Ortmann coll.).

Verbreitung: Vom Rothen Meer bis Natal und Tahiti. - Vom Amboina noch nicht erwähnt.

Chlorodins niger (FORSKAL).

Tgl. Ormaxx, 1. c. p. 465.

Dar-es-Sal a a m, Chokirbank, 203 (OrTMLAxi coll.).

Verbreitung: Rothes Meer und Ost-Afrika bis zu den Paumotu- und Sandwich-Inseln.

\section{Hypocoelus sculptus (MILNE-EDWARDS).}

Cancer sculptus Iruxe-Edwards, Hist. Nat. Crust, I, 1834, p. 37 乃.

Hypocoelus scutptus (M.-E.) Heller, Sitz. Ber. Akad. Wiss. Wien, 43, 1, 1861, p. 322. A. Milde-Edwards, Nouv. Archiv. Mus., I, 1565 , p. 295 . Kossuaxs, Zool. Ergebn. Reis. Roth. Meer, 1, 1877, p. 29. Hilgexporf, Mon. Ber. Ak. Wiss. Berlin, 1578, p. Tis.

D a r-es-Sala am, Upanga-Riff, I J (ORTMAxi coll.).

Ve rbre it un g: Rothes Meer (Milne-Edwards, Heller, Kossuans); Ibo (Hilgendorf); Madagascar:

Nossi Faly (Hoffmann); Cochinchina und Japan (A. Milne-Edwards).

\section{Carpiloules tristis DANA.}

Tgl. Ortulax, 1. c. p. 467.

Dar-es-Sal a am, Chokirbank, I 7 , Ras Rongoni, I $\delta$ (ORTMANx coll.).

Verbreitung: Ceylon (MÜller); Singapur (Ortmans); Australien (Haswell); Neu-Caledonien (A. Milde-Edwards); Paumotu-Inseln (DANA).

\section{Carpiloles vaillantiams A. MILNE-EDWARDS.}

A Milie-Edwards, Nour. Arch. Mus., I, 1865, p. 231, pl. 11, fig. 3. HAinelu, Catal. Austral. Crust., 1882, p. 57. Mifrs, Alert, 1884, p. 529. DE Max, Arch. f. Naturg., 53, 1, 1887, p. 235.

Nach Heller (Crust. Novara, I 865, p. I7) ist diese Art mit Carpilodes rugipes (Helcer) identisch. Nach A. Milxe-EDWARDS sind beide rerschieden. Meine Exemplare stimmen mit vaillantianus, nicht mit rugipes nach der Fassung bei A. MILNE-EDWARDS.

Amboina, I $\delta$ (SEMION coll.).

Dar-es-Salaam, Chokirbank 1 z I $q$ (Ortann coll.).

Verbreitung: Rothes Neer (A. Milis-Edwards); Seychellen (MIERs); Mauritius und Bourbon (A. Milne-Edwards); Java: Ins. Edam und Noordwachter (DE MAN); Amboina (DE Man); Cap Grenville (Haswell); Samoa-Inseln (A. Milne-Edwards).

Crupilodes monticulosus A. MILNE-EDWARDS.

A. Milate-Edwards, Nour. Arch. Mus., 9, 1873, p. 181, pl. 5, fig. 1. de Max, Arch. f. Naturg., 53, 1, 1887, p. 233.

Cephalothorax gelblich, Beine und Scheeren roth, Fingerspitzen weisslich, mit der Beschreibung bei A. Milxe-Edwards übereinstimmend. Dagegen zeigt die Abbildung (1. c.) das ganze Thier dunkelviolett.

Dar-es-Sal a am, Upanga-Riff, I q (ORtuAxN coll.).

Verbreitung: Neu-Caledonien (A. Milne-Edwards); Amboina (de MaN).

Cropilius convexus (FORSKAL).

Vgl. Ortacass, 1. c. p. 469.

Amboina, 4 juv. (Semon coll.).

Dar-es-Sala a m, Chokirbank, $2 \delta$ (Ortuan coll.).

Terbreitung: Rothes Meer und Ost-Afrika bis Tahiti und Sandwich. 
Chlorodopsis melanochirus A. MILNE-EDwaRDS.

Vgl. Ortuann, 1. c. p. 471.

A mboina, 2 o (Semon coll.).

Verbreitung: Java (DE Man); Amboina (DE MAN); Neu-Guinea (Ortmann); Ost-Australien (Hastuell); Neu-Caledonien (A. Milne-Edwards); Fidji-Ins. (Ortmann).

\section{Familie: 0ziislac.}

\section{Daira perlata (HERBST).}

Vgl. Ortmanx, l. c. p. 474 .

A mboina, i $q$ (SEmon coll.).

Verbreitung: Von Mauritius bis zu den Samoa-Inseln. - Von Amboina noch nicht angegeben.

\section{Gattung: Actumuıs.}

Uebersicht der bisher bekannten Arten:

a. Vorderseitenrand in breite Zähne oder Lappen getheilt, nicht granulirt. Cephalothorax oben mit mehr oder weniger deutlich begrenzten Regionen.

b. Vorderseitenrand mit 4 Zähnen hinter der äusseren Orbitalecke. Oberfläche des Cephalothorax fast kahl.

bb. Vorderseitenrand mit drei Zähnen. Oberfläche des Cephalothorax filzig.

c. Scheeren mit dicht gedrängten, schuppenförmigen Tuberkeln besetzt.

cc. Scheeren mit etwas von einander entfernten Höckern besetzt.

bbb. Vorderseitenrand mit zwei Zähnen. Oberfläche des Cephalothorax fein filzig.

A mudus A. M.-E. ${ }^{1}$ ).

A. squamosus (D. H.) ${ }^{2}$ ). A. setifer (D. H.).

A. globulus HeLLER ${ }^{3}$ )

aa. Vorderseitenrand schwach in Lappen getheilt, gleichmässig granulirt, ohne Dörnchen. Oberfläche des Cephalothorax dicht granulirt, Regionen schwach angedeutet.

A. obesus DAN. ${ }^{4}$ ), A. miliaris A. M.-E. ${ }^{5}$ ).

aaa. Vorderseitenrand nicht in Lappen getheilt, granulirt oder mit Dörnchen besetzt. Regionen der Oberfläche völlig unbegrenzt.

b. Vorderseitenrand dornig-granulirt und mit 3-4 grösseren Dörnchen. Oberfläche des Cephalothorax gleichmässig und dicht granulirt, wie die Beine mit langen steifen Haaren besetzt. Stirnlappen von der inneren Orbitalecke durch eine Kerbe getrennt. A. pulcher (MiERs).

bb. Vorderseitenrand mit sechs paarweise stehenden Dörnchen. Cephalothorax nur auf den Anterolateralgegenden mit dornförmigen Körnern. Oberfläche mit kurzen, Beine mit längeren Haaren besetzt. Stimlappen ohne Kerbe in den oberen Orbitalrand übergehend. A. elegans D. M. ${ }^{6}$ ).

Actumnus setifer (DE HAAN).

Vgl. Orturann, Zool. Jahrb., VII, 1893, p. 474.

Thursday Island, I $\delta$ I + (SEMon coll.).

Verbreitung: Mauritius und Seychellen bis Japan und Tahiti.

\section{Actummus pulcher (MIIERS).}

Pilummus pulcher Mises, Rep. Zool. Coll. Alert, 1884, p. 219, pl. 22, fig. A.

Das zweite Glied der äusseren Antennen erreicht den Stirnfortsatz, die Gaumenleiste ist deutlich, reicht bis an den Vorderrand des Mundfeldes, bildet dort aber keine Kerbe, das Abdomen des ơ ist siebengliedrig: also gehört die Art nicht zu Pilummus, sondern zu Actummus.

Thursday Island, I $\delta$ I $q$ (SEmon coll.).

Verbreit ung: Torres-Strasse: Warrior Reef, und Queensland: Albany Island (MIERs).

I) A Milne-Edwards, Ann. Soc. entomol. France (4) VII, IS67, p. 265. DE Man, Journ. Linn. Soc. London, Zool., 22, 1888, p, 49, pl. 2, fig. 4. 5. - Pondichery, Mergui-Inseln.

2) Vgl. Ortmann, Zool. Jahrb., VII, 1893, p. 475. - Japan.

3) Heller, Sitz. Ber. Ak. Wien, 43, I861, p. 54I, pl. 3, fig. 23. A. Milne-Edwards, Nouv. Arch. Mus., I, IS65, p. 286, pl. I8, fig. 4. - Rothes Meer, Nozambique.

4) Dana, U. S. Expl. Exp. I852, p. 244, pl. I4, fig. 3. - Sandwich-Inseln.

5) A. Milne-Edwards, Nouv. Arch. I, 1865, p. 288, pl. 18, fig. 7. - Seychellen. Ist wahrscheinlich mit A. obesus identisch.

6) DE MAN, Journ. Linn. Soc. Zool., 22, I888, p. 47. - Mergui-Inseln. 
Osius rugulosus Stmipson.

Vgl. Ormani, 1. c. 1. 477.

Dar-es-Sa la a m, Cholirbank, I o (ORTMANN coll.)

Verbreitung: Nicobaren bis Tahiti: für Ost-Afrika neu.

Epixanthus corrosus A. MILNE-EDWARDS.

Epixanthus corrosus A. Mulwe-Enwards, Nouv. Arch. Mus., 9, 1873, p. 241, pl. 9, fig. 1. De Man, Arch. f. Naturg., 53, 1887, p. 29', pl. 11, fig. 3. DE Max, Zool. Jahrb., IV, 1889, p. 421. De Mar, Not. Leyd. Mus., 13, 1891, p. 13. Epixanthus nugosus Kossmaxx, Zool. Erg. Reis. Roth. Meer, I, 1877, p. 36. Kossmaxw, Arch. f. Naturg., 44, 1878, p. 253.

Mein Exemplar ist $16 \mathrm{~mm}$ breit und 9,5 $\mathrm{mm}$ lang. Es steht also genau in der Mitte zwischen den Exemplaren von A. Milne-Edwards und DE MAN.

Dar-es-Sal a am, Ras Rongoni, I o (ORTMANN coll.).

Verbreitung: Rothes Meer (Kossmanv); Nadagascar (DE MAN); Sumatra: Padang (DE MaN); Java: Insel Noordwachter (DE MAN); Neu-Caledonien (A. Milne-Edwards).

\section{Epixanthns dentritus (WHTE).}

Vgl. Ortusix, l. c. p. 478.

Dar-es-Salaam, im Hafen bei Mtoni, I (ORTMAnn coll.).

Verbreitung: Mergui-Inseln über die Sunda-Inseln bis zu den Philippinen, Australien und den Fidji-Inseln. - Von Ost-Afrika noch nicht bekannt.

\section{Gattung: Cycloblepas nov. gen.}

Zweites Glied der äusseren Antennen die Stirn erreichend, aber von der Orbita völlig getrennt, da diese letztere völlig kreisfömig durch die Vereinigung des oberen und unteren Randes geschlossen ist. Gaumen mit deutlicher Leiste, die aber nicht ganz bis zum vorderen Rand des Mundfeldes reicht und daselbst auch lieine Kerbe bildet.

Cephalothorax etwas gewölbt, verbreitert, Vorderseitenrand gekerbt, länger als der concave Hinterseitenrand. Oberfläche dicht und deutlich gefeldert.

Gehört zur Unterfamilie Eriphiinae der Ozïdae (vgl. Ortann, l. c. p. 429 und 479). Die zu dieser Unterfamilie gehörigen Gattungen unterscheiden sich folgendermaassen.

a. Gaumenleiste nicht bis zum Vorderrand des Mundfeldes reichend. Cephalothorax quer stark verbreitert, stark gefeldert. Vorderseitenrand gelierbt.

aa. Gaumenleiste bis zum Vorderrand des Mundfeldes reichend und daselbst eine Kerbe bildend. Cephalothorax weniger stark gefeldert.

b. Aeussere Antennen von der Orbita nur wenig entfernt. Cephalothorax etwas quer verbreitert. Vorderseitenrand mit Zähnen.

Eurïppellia MiERs.

bb. Aeussere Antennen von der Orbita weit entfernt. Cephalothorax nicht verbreitert. Vorderseitenrand mit Zähnen oder Dornen.

Eriphic LATR.

Cycloblepas semoni nov. gen. nov. spec. Tafel III, Fig. 8.

Cephalothorax verhältnissmässig wenig gewölbt, nur gegen die Stirn und die Vorderseitenränder abschüssig, I2 mm lang, $20 \mathrm{~mm}$ breit. Stirn leicht gebogen, in der Mitte eingekerbt, die beiden Lappen etwas ausgeschweift. Augenhöhlen völlig kreisrund, oben mit je zwei Fissuren andeutenden Furchen, Ränder sonst ganzrandig. Aeussere Antennen von der Orbita völlig getrennt, ihre freien Glieder fast um $\frac{1}{3}$ des Orbitadurchmessers von den Orbiten entfernt, ihr zweites Glied fest zwischen dem unteren Orbitalrand, dem Stirnrand und den inneren Antennen eingekeilt.

Oberfläche des Cephalothorax durch tiefe Furchen in zahlreiche Felder getheilt. Vorderseitenrand durch kerbenartige Einschnitte in vier Hauptlappen getheilt, diese Lappen sind aber z. Th. wvieder durch schwächere Furchen eingekerbt. Furchen mit dichtem, kurzem Filz besetzt. Die Felder sind auf der Oberfläche dicht granulirt. Hinterseitenrand stark concav.

Erste Pereiopoden gleich gross. Carpus durch unregelmässige Furchen warzig und wulstig, die Warzen und Wülste granulirt. Hand oben ebenso sculptirt, auf der Aussenfläche mit 2-3 Längsreihen von scharfen Körnern. Finger mit Längsreihen von Körnern, schwarz mit stumpflichen Spitzen. Carpus und Hand in den Furchen ebenso wie der Cephalothorax filzig behaart, an den Fingern mit kurzen, steifen Borsten besetzt. 
Hintere Pereiopoden kurz und plump, etwas granulirt und kurz und dicht behaart. Auch die Pterygostomialgegenden sind granulirt. Dritter Maxillarfuss mit fast quadratischem Merus. Abdomen des $\delta$ mit deutlichen Trennungslinien der Glieder, doch sind das 3., 4. und 5. Segment kaum gegen einander beweg. lich. Sternum und Abdomen ebenfalls etwas granulirt und behaart.

Färbung (in Alkohol) ausser dem schmutzig-braunen Filz weisslich mit unregelmässigen, röthlichen Marmorirungen.

Diese Art ähnelt im äusseren Habitus einigermaassen der Actaea rufopunctata, unterscheidet sich aber auf den ersten Blick durch zahlreichere Felder des Cephalothorax, und ferner durch die Gattungsmerkmale.

A mboina, I o (SEMON coll.).

\section{Eurïppellia anmulipes (A. MILnE-EDwards).}

Vgl. Rüppellia annulipes M.-E., ORtmanx, 1. c. p. 479.

Dar-es-Salaam, Ras Rongoni, 3 ơ 2 , Ras Upanga, $\mathrm{I} q$, in altem, mürbem Korallkalk (ORTMANN coll.).

Verbreitung: Seychellen und Amiranten bis Tahiti. - Von der ost-afrikanischen Küste noch nicht erwähnt.

Tgl. Ortmane, l. c. p. 480.

\section{Eriphia laevimana LATREILLE.}

A mboina, I o 2 ? (Senon coll.).

Dar-es-Sala a m, Upanga-Riff, I $q$ (ORTMANN coll.).

Verbreitung: Von Ost-Afrika bis zu den Paumotu-Inseln.

\section{Eriphia smithi MACLEAY.}

E. smithi Macl. Kradss, Südafrik. Crust., 1843, p. 36. Horfuaxx, Crust. Echin. Madagascar, 1874, p. 6, pl. 1, fig. 1.

E. laevimana var. smithi Hılgendorf, Mon. Ber. Ak. Wiss. Berlin, 1878, p. 797. Mrers, Ann. Mag. N. H. (5) T, 1880, p. 237. DE MAN, Arch. f. Naturg., 53, 1, 1887, p. 327.

Kilwa, Amanabank, viele Ex., Dar-es-Salaam, Ras Ndege, 1 q, Ras Chokir, 2 o 3 , Upanga-

Riff, I $\delta$ I $q$ (ORtmann coll.). Ich sah diese Form auch auf der Todteninsel bei Zanzibar.

Port Elisabeth, I o 2 \& (Mus. Strassburg).

Verbreitung: Zanzibar: Ins. Baui (Pfeffer); Mozambique (Hilgendorf, Miers); Ibo (HilgenDORF); Natal (Krauss); Madagascar: Nossi Bé (Hoffmanx); Mauritius (Hilgendorf); Glorioso-Gruppe (Miers); Singapur (Dana, Walker); Java: Ins. Edam und Noordwachter (De Man); Hongkong (Stimpson); Neu-Guinea (Mrers).

Familie: Trapeziidae.

\section{Trapesia cymodoce (HERBST).}

v'(u). typica OrTManN.

Vgl. ORTuan, 1. c. p. 481.

A mboina, $2 \delta 5 q$ (Semor coll.).

Dar-es-S la a m, Upanga-Riff, I $q$, Chokirbank, 4 o 5 , Ras Rongoni, 2 o $2 q$ (Ortmann coll.).

Verbreitung: Die typische Form der Tr. cymodoce ist vom Rothen Meer bis zu den Liu-KiuInseln, Australien und den Marquesas bekannt. - Von Ost-Afrika wird sie angeführt von Zanzibar (PFEFFER) und vielleicht auch von Ibo (HiLGENDORF).

Togl. Ortmann, 1. с. 1. 484.

Tirpesia mofopuctata (HERBST). Form dar.

Meine Exemplare stellen in der Gestalt des Cephalothorax und der Stirnzähne die typische

Amboina, 2 o (Senion coll.).

Dar-es-Salaam, Ras Ndege, I o (ORTMann coll.).

Verbreitung: Bisher nur aus dem Pacifischen Ocean mit Sicherheit bekannt: Java, Philippinen, Neu-Caledonien, Samoa, Tahiti, Marquesas, Sandwich, und bis zur Westküste Mexicos: Socoro-Inseln (A. Milne-Edwards). - Miers (Alert, I884, p. 536) giebt eine Reihe von Fundorten aus dem Indischen Ocean an, doch ist es unsicher, ob dieselben zur typischen rufopunctata oder zur var. maculata gehören. 
Tetralia glaberrima. (HERBST).

Vgl. Ortmann, l. c. p. 485.

Dar-es-Sala am, Upanga-Riff, $2 \delta 2$ (Ortmann coll.).

Verbreitung: Rothes Meer bis Natal und zu den Paumotu-lnseln.

Familie: 'Telphusidae.

Ygl. Ortman, 1. c. p. 487.

\section{Paratelphusa trillentata (MILNE-EDWARDS).}

$P$. convexa DE MAN und $P$. maculata DE MAN möchte ich als Varietäten hiervon auffassen.

Java, Buitenzorg, $3 \delta 5$ (SEmon coll.).

Verbreitung: Sumatra, Java, Borneo, Timor, Bavian, Solor-Insel.

Telphusa (Geotelphusa) kWhli DE MAN.

Vgl. Ortmaxn, l. c. p. 490.

Java, Tjibodas, I of I $q$ (Semon coll.).

Verbreitung: Bisher nur von Java bekannt.

\section{Familie: Grapsillae.}

\section{Metopoyrapsus messor (ForskaL).}

Vgl. Ortuane, Zool. Jahrb., TII, 1894, p. 701.

Die mir vorliegenden Exemplare bilden den typischen MI. messor.

Thursday Island, I $\delta 2$ \& (Semon coll.).

Dar-es-Sala am, im Hafen, I o 2 ( ORTMAN coll.).

Verbreitung: Vom Rothen Neer bis Natal, Tahiti und Sandwich.

Metopograpsus oceanicus (JACQUINOT et LUCAS).

Vgl. De MaN, Arch. f. Naturg., 53, 1, 1887, p. 364 (daselbst die übrige Literatur). - Ferner: Heller, Novara, 1865, p. 44. DE Max, Not. Leyd. Mus., 5, 1883, p. 158.

Dar-es-Salaam, im Hafen, I o (ORTMann coll.).

Verbreitung: Nicobaren (Heller); Java: Pulo Edam und Noordwachter (DE Man); Gebeh und Amboina (DE Man); Pulo Han (Jacqunot et LuCAS).

Grapsus grolısus (LINNÉ).

Vgl. Ortmax, Zool. Jahrb., ViI, 1894, p. 703.

Dar-es-Salaam, Ras Rongoni, i q (Ortuann coll.).

Verbreitung: Im tropischen Indo-Pacifischen und Atlantischen Gebiete, überall. Die Verbreitung ist echt circumtropisch.

\section{Guripsus strigosus (Herbst).}

Tgl. Ortalane, l. c. p. 705.

Dar-es-Sala a m, im Hafen, $2 \delta 6$; Ras Chokir, $6 \delta 4 q$ (Ormann coll.).

Verbreitung: Vom Rothen Meer und Ost-Afrika über das ganze Indo-Pacifische Gebiet bis Chile.

Vgl. Ortuani, 1. c. p. 713.

I'amena litterrata (FABRICIUS).

Dar-es-Salaam, am Eingange des Hafens, an Bimstein, I $q$ (Ortuann coll.).

Verbreitung: Ost-Afrika (Ibo) bis Neu-Caledonien und Neu-Seeland.

\section{Heterograpsus erythrueus (KosSMANN).}

Pseudograpsus erythraeus Kossmanx, Ergebn. Reis. Roth. Meer, 1, 1877, p. 61, pl. 1, fig. 5. Kossmany, Arch. f. Naturg., 44,1878 , p. 255 .

Ist mit $H$. barbimanus HELLER $=$ cremulatus GUÉRIN sehr nahe verwandt: er unterscheidet sich nur durch verhältnissmässig breitere Stirn und völlig glatte Oberseite des Cephalothorax. 
Mein Exemplar trägt nur auf der Innenseite der rechten, grösseren Scheere an der Basis der Finger ein Haarbüschel.

Dar-es-Sala am, im Hafen, I ठ (OrThann coll.).

Verbreitung: Rothes Meer (Kossmann).

\section{Sesarma gracilipes MILne-Edivards.}

Sesarma gracilizes Milme-Edwards, Annal. Sc. Nat. (3), Zool., 20, 1853, p. 182. Jacquinot et Lucas, Yoy. Pole Sud etc., Zool. Crust., III, 1853, pl. 6, fig. 5 (S. impressa juv. in tabulis). Heller, Crust. Novara, 1865, p. 65. DE Max, Not. Leyd. Mus., II, 1880, p. 21. DE Max, Zool. Jahrb., II, 1887, p. 645 u. 663.

S. schiittei Hrss, Decap. Krebs. Ost-Austral., 1865, p. 24, pl. 6, fig. 11*.

Das vorliegende Exemplar, ein + , zeigt keinen deutlichen Höcker auf der Aussenfläche der Hand, sondern nur eine undeutliche, glatte Anschwellung. Im Uebrigen stimmt es vollkommen.

N e u-Guinea, I $q$ (SEMron coll.).

Verbreitung: Madagascar (DE Man); Nicobaren (Heller); Amboina (DE MaN); Neu-Guinea (de Man); Sydney (Hess); Tonga-Inseln, Vavao (Mllne-EdWards, Jacquinot et LuCaS).

\section{Sescrina elongata A. MILNE-EDIVARDS.}

A. Minne-Edwards, Nouv. Arch. Mus,, T, 1869, p. 30. in Mfax, Zool. Jahrb., II, 1887, p. 645. de Mlax, Not. Leyd. Mus., 14, 1892, p. 256.

In die erste Gruppe bei de Man gehörig. Der Oberrand der Palma der Scheeren des o mit einer gezahnten Leiste, die aber genau dem Rande folgt und nicht schräg verläuft wie bei den Arten der dritten Gruppe. Beweglicher Finger oben mit einem fein granulirten Kiel. Meren der hinteren Pereiopoden stark verbreitert. Cephalothorax länger als breit, bei meinem o $25 \mathrm{~mm}$ lang, $23 \mathrm{~mm}$ breit, beim $q 28 \mathrm{~mm}$ lang, $25 \mathrm{~mm}$ breit: die Breite ist an den äusseren Orbitalecken gemessen.

Dar-es-Sala am, am Hafen, i o I q (OrTMAN coll.).

Verbreitung: Westküste von Madagascar (A. Milne-Edwards).

Ygl. Ortmana, 1. c. p. T20.

Sesarma meimerti DE MAN.

Das eine $\delta$ aus dem Upanga-Thal zeigt Spuren eines zweiten Epibranchialzahnes.

Dar-es-Salaam, am Hafen, oberhalb der Flutlgrenze, $2 \delta$ I $q$, in den Süsswassersümpfen des Upanga-Thales, 2 (OrTMary coll.).

Verbreitung: Von Ost-Afrika (Zanzibar bis Natal) bis Neu-Caledonien.

Sescrma nodulifera DE MAN.

Sesarma (Geosesarma) nodulifera Ie Max, in: Werer, Zool. Erg. Reis. Niederl. Ost-Indien, II, 1892. p. 34:, pl. 20, fig. 16 .

Die Untergattung Geosesarma wird sich kaum halten lassen.

Java, Buitenzorg, I o (SEMron coll.).

Sesarma (Geosesurma) sp. DE MAa, ibid., p. 840.

var. conferta not:

Die vorliegenden Exemplare sind sicher mit der von DE MIAN nicht näher benannten Form identisch. Auch sämmtliche mir vorliegenden Exemplare sind klein und unterscheiden sich von typischen Exemplaren durch etwas zahlreichere und dichter gedrängte Körnchen auf dem Rücken des beweglichen Scheerenfingers. Ich fasse diese Form als Varietät von nodulifera auf.

Java, T jibodas, 4 đ 5 ? (Semon coll.).

Verbreitung: Die typische Form stammt von Buitenzorg (DE MaN). Von dieser finden sich im Mus. Strassburg Exemplare von Java: Tjisurupan, 4000 Meereshöhe. Die var. conferta findet sich in Java bei Tjibanas und Tjibodas ( $\mathrm{DE}$ MAN).

\section{Sesarma erythrodactyla HESs. \\ var. africana nov.}

S. quadrata Hilgendorf, v. d. Decken's Reisen. III, 1, 1869, p. 90, pl. 3, fig. 3c; pl. 4, fig. 3. Hoffmanx, Crust. Echinod. Madagascar, 187t, p. 23.

Stimmt mit den mir vorliegenden Exemplaren der S. erythrodactyla von Sydney und Japan recht gut überein, besonders was die Anzahl der Querwülste auf dem beweglichen Scheerenfinger, und was die

Gestalt und Grösse des Cephalothorax und die Gestalt der Beine anbelangt. 
Sie unterscheidet sich jedoch durch die schwache Entwickelung der gekörnten Leiste auf der Innen. seite der Palma, die nur beim $\delta$ noch erkenubar, aber da viel weniger ausgebildet ist als bei typischen Exemplaren.

Der Merus der Scheerenfüsse besitzt keinen Dorn am inneren Rande, sondern zeigt dort nur einen gerundeten, gezähnten, schwach vorspringenden Lappen. Bei der typischen erythrodactyla findet sich dort ein dreieckiger, gezïhnter Lappen. Alle diese Merkmale werden von HilgENDorf ausdrücklich angegeben.

Ferner sind die Krallen etwas länger als bei den typischen Exemplaren. fast so lang als der Propodus.

Färbung der Hand röthlich, der Finırer weisslich, bei erythrodactyla typ. umgekehrt. Die von DE MAN (Not. Leyd. Mus., I2, I890, p. I00) für erythrodactyla angegebene granulirte Linie in der Mitte der Aussenfläche der Hand finde ich ebenfalls bei typischen of Exemplaren von Sydney; bei einem (jüngeren) ठ von Japan ist sie kaum angedeutet. Den typischen $q$ von Sydney fehlt sie, und bei der afrikanischen Form fehlt sie dem $\delta$ und dem ?.

Auf dem Oberrand der Hand findet sich bei den afrilianischen Exemplaren nur e in e braune Kammleiste, nahe der Basis des beweglichen Fingers, aber in derselben Richtung laufend, wie bei typischen Exemplaren.

Mikindani, im Sumpf hinter dem Stationsgebäude, I +. Dar-es-Salaam, am Hafen, $2 \delta 2$ 웅 (ORTMAnN coll.).

Verbreitung: Die typische erythrodactyla ist bekannt von Sydney (Hess) und Japan (OrTmann) die var. africana wird von Zanzibar (HLlgENdorf) angegelen, sowie von Madagascar: Nossi Faly, Nossi Bé und Sakatia (HoffMans). - Vielleicht gehört auch das von DE MAN (Zool. Jahrb., IV, I889, p. 434) von Madagascar erwälınte q zu dieser Varietät.

\section{Helice lertelli Hess.}

Melice leachi Hess, Decap.-Krebse Ost-Austral. 1sij. p. 27. ne Max, Znol. Jahrb., II, 1887, p. 702.

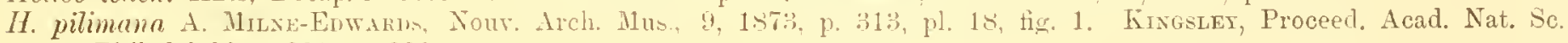
Philadelphia, 1880 , p. 2:0.

Dar-es-Sala am, am Hafen, I 7 (ORTMANn coll.).

Verbreitung: Sydney (HEss); Neu-Caledonien (A. Milne-Edwards).

I'gl. Ortmans, l. c. p. 729.

Cyclo!gulusus pumetutus MiLnE-EDWARDS.

Die vorliegenden Exemplare sind typisch.

Port El is a beth, $3 \delta$ (Nus. Strassburg). Hongkong.

Verbreitung: Süd-Afrika, Tasmanien, Australien, Neu-Seeland. Ferner: Neu-Guinea und

\section{Playmsia immenculuto LAMARCK.}

Tgl. Ontmain, 1, c. p. 730.

Mikindani, an einer Boje vor dem Eingang zum inneren Hafen, I of I $q$ (OrTMANN coll.).

Verbreitung: Ost-Indien bis zur Westküste Amerikas. - Neu für Ost-Afrika, die nächsten Fundorte sind: Malabar MilnE-Edwards) und Ceylon (Miers).

\section{Leiolopleles abbreviatus (DANA).}

Tgl. Miers, Annal. Mag. N. H. (5), I, 1s78, p. 154 (daselbst die ältere Literatur) dr Man, Arch. f. Naturg., 53, 1887, p. 372. Thaldwitz, Abh. Mus. Dresden, 1891, p. 36.

A mboin a, I $\delta$ (SEMON coll.).

Milindani, mit der vorigen Art, I $\delta 2$; Dar-es-Salaam, Upanga-Riff, 2 q (ORTMANN coll.).

Verbreitung: Mauritius (Mrens); Molukken (Miers: Dj_lolo (Miers), Amboina (DE MaN); Philippinen (Miers): Guimaras (Miers); Flores (Thallwitz); Timor (Thallwitz); Allor (Thallwitz); Fidji-Ins. (MIERs); Tahiti (DANA).

Familie: Gecarcinidae.

\section{Cardisoma calnifex (HERBst).}

Tul. Ortmans, Zool. Tahrb., VII, 1894, p. 735.

Lindi. oberhalb der Mangroven, I Dar-es-Sala am, oberhalb der Flutgrenze, I $\delta 2 \delta$ (ORTMANN coll.).

Verbreit ung: Von Ost-Afrika bis zu den Paumotu-Inseln.

Jenaische Denkschriften VIII. 
Familie: Oeypodidar.

\section{Euplax boseii (AUdoun).}

Macrophthalmus boscii Acn., Krauss, Südafrik. Crust., 1843, p. 40, pl. 2, fig. 5.

Euplax boscii (Aud.) Milne-Edwarus, Annal. Sc. Nat. 3, Zool., 18, 1852, p. 160. A. Mulate-Edwards, Nont. Arch. Mus., 9, 1873, p. 281. me Mas, Not. Leyd. Mus. II. 1880, p. 71. Miers, Alert, 1884, p. 540. Miers, Chall.

Brach. 1886, p. 252. DE MAx, Arch. f. Naturg., 53, 1887, p. 357.

Chaenostoma orientale Strmpsos, Proceed. Acad. Nat. Sc. Philadelphia, 1858, p. 97.

Dar-es-Salaam, am Aussenstrande, auf felsigem, von todtem Korallkalk gebildetem Grunde, viele Ex. (ORTMANn coll.)

Verbreitung: Rothes Meer (MILne-Edwards); Zanzibar (Milne-Edwards); Mozambique (Miers);

Natal (Kratss); Madagascar: Nossi Bé (Lenz und Richters); Java: Insel Noordwachter und Edam (DE MAN): Celebes (DE MAN); Amboina (DE MaN); Liu-Kiu-lnseln (Stimpson); Neu-Caledonien (A. MıneEDwards); Fidji-Ins. (DE MaN, Miers).

\section{Macrophthalmels telescopicus (OWEN).}

Tgl. Ortmann, 1. c. p. 74.

Thurs day Is land, I $q$ (SEMON coll.).

Verbreitung: Sandwich-Inseln (Randall, Milne-Edwards, Dana, Eydoux et Souleyet); LiuKiu-Ins. (Stimpson); Carolinen (Ortmann); Torres-Strasse (Miers); Port Denison (Haswell).

\section{Macrophthalmurs gramlialieri A. MLNE-EDWARDs.}

M. grandidieri A. Muse-Edwards, Nonv. Arch. Mis., IT, 1868, p. 84, pl. 20. fig. 8-11. Lexz und Richters. Beitr. Krust.-Faun., Madagascar, 1881, p. 3.

M. brevis Hiliexmorf, v. d. Decken's Reisen, 1869 , p. 68, pl. 3. fig. 4. De Mas, Not. Leyd. Mus., II, 1880, p. 70.

M. carinimanus Hilgextorf, Mon. Ber. Akad. Wiss. Berlin, 1878. p. 806.

Mit Hilgendorf's Art völlig übereinstimmend und ebenso mit M. grandidieri bei A. Milne-EDwards.

Die Beschreibung des Letzteren ist insofern etwas unklar, als die Zähne an der äusseren Orbitalecke abweichend gedeutet werden: ihre Anordnung ist aber genau dieselbe wie bei HiLGEndorf's Art und für diese Art charakteristisch.

Nach Hilgendorf (I878) ist diese Art verschieden von $M$. brevis Herbst. Nach de Man ist sie auch mit carimimanus nicht identisch.

Kilwa, am Strande, I $d$. Dar-es-sala am, im Hafen bei Itoni und an Aussenstrande, viele Ex. (ORTMANN coll.)

Lebt innerhalb der Ebbezone, wo er sich in schlammig-sandigem Grunde Löcher gräbt.

Verbreitung: Rothes Meer (Hilgendorf); Zanzibar A. Mllne-Edwards, Hilgendorf); Mozambique (Hilgendorf); Madagascar: Pasandava-Bai (DE MAN), Nossi Bé Lenz und Richters).

\section{Dotilla fencstrata HILGENDORF.}

Tgl. Ortmaxi, 1. c. p. 748

Kilwa, am Strande, 7 Ex.; Dar-es-Sala am, am sandigen Strande des Hafens, viele Ex. ORTMANN coll. .

Verbreitung: Zanzibar Pfeffer); Ibo (Hilgendorf); Mozambique (Hilgendorf, Miers ; lnhambane (HılgendrF); Natalbai Krauss).

\section{Hyctiris longicorpus LATREILLE.}

Ygl. Ortann, 1. c. p. 748.

A mboin a, 16 \& 7 (SEMON coll.).

"Diese Krabbe wirft zur Ebbezeit den Seesand am Strande in kleine Haufen auf, dass der Strand stellenweise ein Aussehen bekommt, als sei er gepflügt" (SEMON).

Verbreitung: Australien (Mllne-Edwards, Daxa, Stimpson, Heller, Haswell, Miers; NeuCaledonien (A. Milwe-Edwards); Neu-Guinea (Mlers); Timorlaut Miersı; Amboina (de Max); Philippinen (Miers); Liu-Kiu-Ins. (STiupson); China (Stmpson, Ortuane); Singapur (Ortuann).

Gelesimus cultrimanels WHHTE.

Firl. Ortmans, J. c. p. 753

Auch unter diesem Naterial liegen mir verschiedene Formen der Ausbildung der Zähne am un- 
beweglichen Scheerenfinger vor, wie sie Thallwitz geschildert hat. Die in der Tabelle bei DE MaN und von mir angegebenen Merkmale sind aber auch hier constant.

Li ind, unterhalb der Mangroven, $9 \delta 5 \%$ Kilwa, am Strande, 7 I $q$; Dar-es-Sala am, am Hafen, I o (ORTMANn coll.).

Verbreitung: Ost-Afrika bis zu den Liu-Kiu- und Samoa-lnseln.

\section{Gelasimus meillei MuLne-EDWARds.}

G. urvillei Milse-Emwards, Annal. Sc. Nat. (3, Zool., 18, 1853, p. 148, pl. 3, fig. 10. Kixgsler, Proceed. Acad. Nat. S̈. Philadelphia, 1880, p. 145. we Max, Not. Leyd. Mus., 13, 1891, p. 34.

G. dussumieri Hilgeninurf, v. d. Decken's Reisen, III, 1, 1869, p. 84, pl. 4, fig. 1. Hoffmans, Crust. Echinod. Madagascar, 1874, p. 17 (z. Th.) ${ }^{1}$ ). Pfeffer, Jahrb. Hamburg. wiss. Anstalt, TI, 1889, p. 30.

Meine Exemplare sind identisch mit HulgendorF's dussumieri und mit urvillei nach der Fassung bei DE $M$ AN

Farben im Leben: Cephalothorax lebhaft blau, grosse Scheere roth oder gelb.

Lindi, in den Mangroven, im schwarzen Schlamme, I J; Dar-es-Salaam, in der Bucht bei Mtoni, im schwarzen Mangrovenschlamme, 9 d 2 q (Ortmann coll.).

Verbreitung: Zanzibar (Hılgendorf); Bagamoyo: Kingani (Pfeffer); Madagascar: Nossi Bé (HoffmanN) ${ }^{2}$ ); Mergui-Ins. (DE MaN); Vanikoro (Milne-EdWards).

\section{Gelasimms imerems HoFFmanN.}

(t. inversus Hofranx, Crust. Echinod. Madagascar, 1874, p. 19, pl. 4, fig. 23-26. De Man, Not. Leyd. Mus., 13, 1891, 1. 44, pl. 4, fig. 12 .

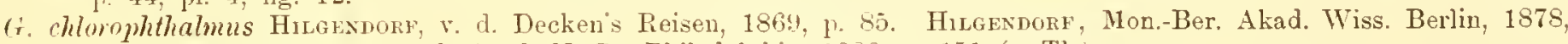
p. 803. Kixqsuly, Proceed. Acad. N. Sc. Philadelphia, 1880, p. 151 (z. Th.).

Gel. inversus ist eine gut charakterisirte Art.

Lindi, oberhalb der Mangroven, I $\delta$; Dar-es-Salaam, in der Bucht am alten Sultanspalast, I $\delta$; ferner wurden mir 3 \& $q$ mit l'enaeus-Arten, Leander longicarpus, Caridina wycki etc. gebracht, wohl von der Upanga-Mündung.

Verbreitung: Mozambique (Hilgendorf) ${ }^{3}$ ); Madagascar: Nossi Faly (Hoffmann). - Weitere Daten sind nicht bekannt, da die übrigen Angaben (bei KINGSLEr) sich nicht mit Sicherheit auf diese Art beziehen lassen.

\section{Gelasimus ammulipes MuLne-EDWARDS.}

Trgl. Ortuaxi, l. c. p. 758 .

Farbe im Leben grau, mit dunkleren Flecken, grosse Scheere weisslich oder röthlich.

Zanzibar, in der Lagune hinter der Stadt, 3 j; Lindi, oberhalb der Mangroven, im Sande, 4 ; Kilwa, am Strande in den Mangroven, an sandigen Stellen, viele $\delta$ und $q$; Dar-es-Sala a m, an der evangelischen Mission, zwischen Sand und Steinen, I2 84 ; in der Bucht am alten Sultanspalast, im Sande, $9 \delta 9$ \% Ortmann coll.).

Verbreitung: Vom Rothen Neer und Ost-Afrika über das Indo-Pacifische Gebiet bis Tahiti und Samoa.

\section{Oeypode lillli DE HAAN.}

I): HaAN, Faun. Japon., 1850, p. 58. DE MaN, Not. Leyd. Mus., III, 1881, p. 250. Miers, Ann. Mag. N. H. (5), I, 1882, p. 384, pl. 17, fig. 8, Sa. Minrs, Alert, 1884, p. 237. Prefrer, Jahrb. Hamburg. wiss. Anstalt., 1889, p. 30. De Man, Not. Leyd. Mus., 15, 1893, p. 286.

Steht am nächsten der 0 . pygoides Ortmans. Stimmleiste vorhanden, nur aus Körnern gebildet, bei meinen Exemplaren aus 8-I3 Körnern bestehend (DE MAN giebt 8-IO, MiERs deren I7 an). Unterer () rbitalrand mit äusserer und mittlerer Einkerbung, beide jedoch nicht so tief wie bei pygoides. Von letzterer unterscheidet sie sich wesentlich durch das Fehlen der Haarbürsten auf den 2. und 3. Pereiopoden.

Songa-Songa-Insel (bei Kilwa), 3 ¿ 3 ; Kilwa, am Strande, 2 o; Dar-es-Salaam. am Aussenstrand, I 3 (ORTMANN coll.).

Port Elisabeth, $5 \delta 2$, alle klein oder mittelgross (Mus. Strassburg).

1) Die von Hoffmans erwähnten Exemplare gehörell z. Th. auch zum echten $G$. dussumitri, vgl. DE MAN, 1. C. p. 26.

2) Nossi Faly bei DE MAN wohl per errorem, da es dieselben Exemplare sind, die Hoffmans vorlagen.

3) Hilgend orf giebt nirgends ,Zanzibar" an, wie KingsLeY behauptet. 
Verbreitung: Zanzibar: Ins. Changu und Baui (Pfeffer:; Madagascar (Miers); West-Australien: Shark Bay (Miers); Java (DE MAN); Japan (Miers); Torres-Strasse: Thursday Island (MiEns); Neue Hebriden (MIERs); Sandwich-Inseln (Miers).

\section{Ocypode ceratophthalma (PALLAS).}

Vgl. Ortmann, 1. c. p. 767 .

Thursday Island, 1 juv. (SEMon coll.).

Songa-S onga-Insel, I $q$ ad. (mit Augenhörnern); Kilwa, am Strande, 2 juv. (mit kaum angedeuteten Augenhörnern); Dar-es-Sala am, Strand am Eingange des Hafens, viele Ex. (nur die erwachsenen mit gut ausgebildeten Hörnern) (ORTMANN coll.).

Port Elisabeth, I $q$ juv. (Mus. Strassburg).

Verbreitung: Vom Rothen Meer bis Natal und zu den Sandwich-Inseln.

\section{Stomatopoda.}

\section{Lysiosquilla maculata (FABRICIUS).}

Vgl. Mrers, Ann. Mag. Nat. Hist. (5) T, 1880, p. 5, pl. 1, fig. 1, 2.

Amboina, I $q$ (SEMON coll.).

Verbreitung: Indo-Pacifisches Gebiet, Mascarenen bis Sandivich und Samoa.

Pseudosquilla om mer MIERS.

Vgl. Miers, ibid. p. 111, pl. 3, fig. 5-6.

Amboina, I o (Semon coll.).

Verbreitung: Philippinen (Mlers); Amboina (DE MaN); Süd-Japan: Kagoshima (Mus. Strassburg); Samoa-Inseln (Mus. Strassburg).

\section{Gomodretylus scyllarus (LINNE).}

Vgl. Mrens, ibid. p. 115.

Amboina, I o (SEMon coll.).

Verbreitung: Indo-Pacifisches Gebiet von Zanzibar und Madagascar bis Samoa.

\section{Gonodactylus chiraghen (FABRICIUS).}

Vgl. Mrers, l. c. p. 118. Brooks, Challeng. Stomatop., 1886, p. 56, p1. 15, fig. 4. A mboina, 4 o 6 (Semon coll.).

Dar-es-Salaam, in Korallkalk, 3 i i juv. (OrTMann coll.).

Verbreitung: Ueberall im Indo-Pacifischen Gebiete. - Die atlantische Form, die hierher gehören soll, ist, wie mir Herr Dr. HANSEN in Kopenhagen mittheilte, hiervon verschieden.

\section{Gonorlretylus glriber (Brooks) ${ }^{1}$ ).}

G. ylabrous Brooks, Chall. Stomatop., 1886, p. 62, pl. 14, fig. 5: pl. 15, fig. $7,9$.

Da r-es-Sal a m, Upanga-Riff, I $q$, Chokirbank, 2 juv. (ORTMAns coll.).

Verbreitung: Häufig vom Rothen Meer bis Nord-Australien (Hansen).

\section{Gomorlactylus grophurus WHITE ${ }^{1}$ ).}

Vgl. Miens, Ann. Mag. (5) T, 1880, p. 120, pl. 3, fig. 9.

Miers hat, wie mir Hansen mittheilt, diese Art nicht klar erkannt.

Thursday I sland, I of I \& (SEMION coll.).

Verbreitung: Australien (Hansen).

I) Die Bestimmung der beiden Arten G. glaber und graphurus wurde mir ermöglicht durch die Angaben. die mir Herr Dr. HANSEN in Kopenhagen, der eine Revision der Stomatopoden vorbereitet, auf meine Anfrage bereitwilligst übersandte. lch nehme hier die Gelegenheit wahr. Herrn Dr. HANSEN für seine liebenswürdige Unterstützung öffentlich meinen Dank auszusprechen. Auch die Angaben über die Verbreitung dieser beiden Arten verdanke ich seinen Mittheilungen. 
Conorluetylus nor: spec.

Diese Art wird von Herrn Dr. Hansen beschrieben werden.

Dar-es-Salaam, Upanga-Riff und Chokirbank, $4 \delta 4$ (ORTMann coll.).

Gonorlactylus trispinosus WHITE.

var. mulchrlla MiERs.

G. trispinosus mulchellus Miess, 1. c. p. 122.

Protosquilla trispinosa (Wн.) Broors, 1. c. р. 71.

Mittlerer Dorn der Rostralplatte gut entwickelt, wie beim Typus. Das Exemplar, das Miers von dieser var. vorlag, zeigte diesen Dorn abgebrochen.

Dar-es-S al a a m, Upanga-Rift, I q (ORTMANn coll.).

Verbreitung: typ.: West-Australien: Swan River und Shark Bay (Miers); Mauritius HoffmanN); Amboina (Miers); Auckland (Heller); Fidji-Inseln (DAnA); - var. pulchella: Ceylon (Miers).

Gomoductylus stolim?s (MÜLLER).

Protosquilla stoliura Múlder, Terh. Naturf. Gesellsch. Basel, Bd. 8, Heft 2, 1887, 1'. 477, pl. 4, fig. 2. IIE Mran, Arch. f. Naturg., 53, 1, 1887, p. 576 .

Amboina, 2 (SEMon coll.).

Verbreitung: Bisher nur von Amboina bekannt.

\section{Cirripedia.}

Familie: Lepadidae.

\section{Lcpas anatiferor LINNÉ.}

Vgl. Darwix, Monogr. Cirripedia, I, 1851. p. 73, pl. 1, fig. 1. Hoek, Challeng. Cirriped, 1883, p. 38, pl. 1, fig. 2. Amboina, 2 Ex., mit der folgenden Art (SEmon coll.).

Mikindani, an der Boje, viele Ex., Dar-es-Salaam, an schwimmendem Holz, viele junge Ex. (ORTMANN coll.).

Verbreitung: An schwimmenden Gegenständen, kosmopolitisch.

\section{Lepres alserifera LINNÉ.}

Tgl. Darwis, l. c. p. 81, pl. 1, fig. 4. Hokr. 1. c. p. 39.

Amboina, viele Ex.'1, S.W.Celebes, Palostai, an Holz, viele Ex. SEmon coll.'.

Verbreitung: Kosmopolitisch.

Familie: Balanidae.

Balanus tintimnabulum LINNÉ).

"ar. oecator" DARWIN.

T'gl. Darwis, Monogr. Cirriped., II, 1854, p. 196, pl. 1, fig. k; pl. 2, fig. $1 \mathrm{~b}$.

Mikindani, an der Boje, viele Ex. (Ortmann coll.).

Verbreitung: $B$. tintimabulum ist kosmopolitisch mit Ausnahme der kälteren Meere, die var. occator giebt DaRwin aus der Südsee an.

\section{Tetraclita porosa (LINNÉ).}

Tgl. Darwix, l. c. p. 329, pl. 10, fig. 1 .

Amboina, viele Ex. SEMON coll.)

Mikindani, an der Boje, viele, meist junge Ex.; Dar-es-Salaam, Chokirbank, an altem Korallkalk in der Ebbezone, viele Ex. (ORTMANN coll.).

Verbreitung: Circumtropisch.

I) Eine Anzahl auf und in Sptrula-Schalen. 
Tetraditu coemulescens (SPENGLER).

Vgl. Darwin, l. c. p. 342, pl. 11, fig. 4. Ноек, Chall. Cirrip., 1883, p. 161, pl. 13, fig. 34.

Thursday Island, viele Exemplare (SEmon coll.).

Verbreitung: Pacific, Philippinen (Darwin), Samboangan (Hock).

\section{Pyigoma cancellatum LEACH.}

Vgl. Darwin, l. c. p. 362, pl. 12, fig. 5 .

Thursday Island, mehrere Exemplare, eingewachsen in eine Steinkoralle, Turbinaria crater (SEMON coll.).

Verbreitung: Ost-Indien (bei DARwis, l. c. p. 363 per errorem West-Indien).

Pyrgoma milleporue DaRWIN.

Vgl. Darwin, l. c. p. 367, pl. 13, fig. 2.

D a r-es-S a la m, Upanga-Riff, viele Exemplare, eingewachsen in Millepora platyphylla var. verucosa (OrTMlann coll.).

Verbreitung: Philippinen (DARWIN).

\section{Pyenogonida.}

Phoxichilus meridionalis Вӧнм.

Bӧнм, Mon. Ber. Akad. Wiss. Berlin, 1879, p. 189. pl. 2, fig. 4.

Meine Exemplare weichen von der Beschreibung und Abbildung bei BöHs nur dadurch ab, dass der Körper keine ,perlartigen Wärzchen“ zeigt, und dass die kräftigen Stacheln in der Mitte der 4. Glieder der Beine, die Böнm fig. 4b zeichnet, fehlen.

Mikindani, an der Boje, zahlreiche Exemplare (OrTMann coll.

Verbreitung: Singapur und Tur am Sinai (Böнм). 


\title{
Biologisehe und bionomische Beobachtungen.
}

\author{
Gattung: Penaeus.
}

Die Arten dieser Gattung leben rein nectonisch, scheinen echt marin und litoral zu sein, dringen aber häufig in Aestuarien ein. Die Exemplare, die ich selbst sammelte, stammen alle aus reinem Salzwasser. Interessant ist es, dass ich zusammen mit P. monoceras und indicus einige Süsswasserformen (Caridina wycki und Palaemon-Arten) und Strand- Brackwasser-Formen (Gelasimus) erhielt neben anderen Salzwasserformen, wie Leander longicarpus). Die Localität, von der diese alle stammen, wird wohl die Mündung des UpangaFlusses sein, etwas nördlich von Dar-es-Salaam, wo Süsswasser und Salzwasser zusammentreffen, etwa in ähnlicher Weise, wie in der Bucht bei Mtoni. An letzterer Stelle sah ich in $\mathrm{I}-2 \mathrm{~m}$ tiefem, reinem Salzwasser zahlreiche Penaeen schwimmen, von denen ich einen ( $P$. monodon) erbeutete. Ganz dicht dabei findet sich Brackwasser und Süsswasser, in den Mangrovesümpfen.

Familie: Atyidae.

Sind durchweg, wie schon längst bekannt, echte Süsswasserformen.

Familie: Alpheilae.

Besteht im Wesentlichen aus Korallriff bewohnern. In dem fein verzweigten Astwerk lebender Korallen fand ich: Alpheus macrochirus, A. laevis, A. gracilipes. Andere Arten, wie auch macrochirus und laevis fand ich in todtem, porösem Korallkalk. A. edwrerdsi wie auch der vielleicht als ơ zu edwardsi gehörige, A. lobidens kommen auch auf anderen Facies vor vgl. oben). Mit den Scheerenfingern verursachen die Arten der Gattung einen knipsenden Laut. Athanas dimorphus fand ich in todtem Korallkaik.

Saron marmoratus lebt zwischen lebenden und todten Korallen, in grösseren Löchern und Höhlungen.

Pontonia pimae schmarotzt im Innern einer Pinna-Art.

Coralliocuris und Anchistia leben zwischen todten Korallen.

Leander longicarpus ist eine nectonische Form: ich fand sie auf felsigem Grunde in grösseren Wasserlöchern schwimmend.

Die Arten der Gattung Palaemon sind, wie längst bekannt, Süsswasserformen, finden sich jedoch bisweilen in Brackwasser und selbst in reinem Salzwasser '1.

Die Gattung Stenopus lebt nach Broors und Herrick (Mem. Nat. Acad. Sc., Y, t, I892) nectonisch auf und in der Nähe von Korallriffen.

Thalassina anomala lebt nach MIERS (vergl. oben) in Mangrovesümpfen, nach GRÄFFE2) hält sie sich in Schlamme grosser Brackwassersümpfe auf.

I) Vgl. Ortmanx. Zool. Jahrb., V. I891, p. 744. - ORtmann, Decap. u. Schizopod. d. Plankton-Exp., 1893. p. 48.

2) GRÄFFE, Notizen über die Fauna der Viti-Inseln. - Verh. Zuol. bot. Gesellsch. Wien, 16, I866, p. 587. - Vgl. auch StUder, Gazelle, III, I889, p. I93. 


\section{Familie: Polcellanidae.}

Alle von mir gesammelten Porcellaniden sind Riffbewohner und halten sich in den Löchern und Höhlungen besonders des todten Korallkalkes auf: man erhält sie gewöhnlich beim Zerschlagen des letzteren. Sie scheinen aber nicht an die Korallfacies selbst gebunden zu sein, sondern an einen felsigen, höhlenreichen Untergrund.

Die Arten der Gattung Pagurus leben auf Korallriffen, scheinen aber nicht an die Korallfacies sich zu binden, sondern an die Tang- und Seegraswiesen, die sich dort finden.

Die Clibanarius- und Calcinus-Arten kommen auf verschiedenen Facies vor. Calcinus herbsti ist für felsigen Strand charakteristisch.

\section{Familie: Coenobitidae.}

Die Coenobitidae sind echte Landkrebse, von der Gattung Birgus und auch von Coenobita ist das längst bekannt ${ }^{1}$ ). So viele Exemplare der letzteren Gattung ich erbeutete, so habe ich doch niemals eines im Wasser gesehen, und ich bezweifle überhaupt, dass sie je auf längere Zeit das Wasser aufsuchen. Man findet sie häufig am Strande, wo sie ihre Nahrung zur Ebbezeit unter den zurückbleibenden organischen Stoffen suchen. Bemerkenswerth war es mir, dass ich nur zur Regenzeit (Ende December und Anfang Januar) Coenobiten umherlaufen sah. Zur trockenen Jahreszeit vermisste ich sie: es wurden mir jedoch zahlreiche Exemplare von Negern gebracht. die sie, nach ihrer Aussage, unter todtem Laube unter Mangobäumen hervorgesucht hatten. Diese Angabe schien mir anfangs etwas zweifelhaft, bis ich selbst eine Anzahl derselben fand, und zwar unter einem grossen Stein am Fusse eines Mangobaumes in Gesellschaft von Iuliden und Scorpionen ${ }^{2}$ ). Es wird mir dadurch wahrscheinlich, dass sie die trockene Jahreszeit, in derartigen Schlupfwinkeln ruhend, überdauern.

Die beiden Formen von Dromiidre, die ich erbeutete, kriechen langsam und träge zwischen Seegras und Tang auf den Riffen herum. Da sie ihren Rücken mit Schwämmen u. dergl. bedecken, sind sie schwer aufzufinden. Sie scheinen an das Vorkommen von Tang- und Seegraswiesen gebunden zu sein.

Calappa hepatica und Matuta victrix kommen bei Dar-es-Saiaam unter denselben Bedingungen vor. Beide halten sich im Hafen auf sandigem Grunde in der Nähe des Strandes auf, wo sie sich durch ihr lebhaftes und rasches Hin- und Herschwimmen bald bemerkbar machen. Während die Bewegung der Calapu mehr ein seitliches Gleiten ist, indem die Füsse eben noch den Sandboden berühren, arbeitet sich Matuta durch kräftige Schwimmbewegungen der beiden hinteren Beine weiter, indem sie jedoch ebenfalls stets dicht über den Sandgrund hinhuscht. Bei drohender Gefahr graben sich beide mit grosser Schnelligkeit in den Sand ein, aus dem sie dann nur die Augen herausstrecken. Gefangen lässt Matuta durch abwechselndes Reiben der Scheeren an der Pterygostomialgegend ein schnarrendes Geräusch ertönen. Ausserhalb des Wassers sterben beide ziemlich schnell ab. Für die indo-pacifische Region sind sie äusserst charakteristische Formen.

I) Vgl. u. a. z. B. Brock, Zool. Jahrb., II, 1857, p. 979. - In der „Bionomie des Meeres", p. 95 und r74, scheint es IVALTHER unbekannt zu sein, dass es unter den "Bernhardkrebsen" echte Landkrebse giebt, während er in der "Lebensweise der Seethiere", p. 526 u. 527 von „Landanomuren" spricht und auch die Gattung Coenobua als am Strande des Rothen Meeres lebend erwähnt.

Die Stelle p. 174 in der "Bionomie" ist noch besonders deshalb interessant, weil dort eine ganz eigenthümliche Charakterısirung des marinen Lebensbezirkes der „oceanischen Archipele“ gegeben wird. Um diesen 1. c. p. r5 aufgestellten Lebensbezirk von anderen zu unterscheiden, führt WALTHER besonders p. 173 u. 174 eine Reihe von Eigenthümlichkeiten auf: alle sind aber Erscheinungen, die sich an jeder beliebigen Continentalküste gleicher Weise und oft besser beobachten lassen. Um dann zu belegen, „dass gerade auf Inseln litorale Thiere leicht festländische Lebensweise annehmen“, führt er drei Beispiele an. Erstens die Bernhardskrebse auf Neu-Hannover, die auf dem Lande herumlaufen. Ausser an der citirten Stelle wird diese Thatsache in Gazelle, III, p. 540 erwähnt, wo die Gattung roenobita genannt wird, die nicht nur auf Inseln, sondern auch an festländischen Küsten auf dem Lande lebt. Was die beiden weiteren Beispiele für den obigen Satz beweisen sollen, ist mir völlig unklar geblieben: wie kann das Laichen der Fische im Kratersee von St. Paul (vgl. Gazelle, I. p. I34, und III, p. I77) und das Vorkommen von Krebsen daselbst (nicht das „Laichen" derselben, l. c. ist lasus lalandei gemeint) genannte Thiere veranlassen aufs Land zı gehen? Schliesslich was die Meeresschildkröten betrifft, so besuchen diese Inseln und continentale Küusten (z. B. Portugal Afrika, Brasilien zur Eiablage, es fällt ihnen aber gar nicht ein, sich deshalb an festländische Lebensweise zu gewölınen. Auch dürfte die Annahme einer eventuellen Ableitung der Landschildkröten von Seeschildkröten nicht den phylogenetischen Thatsachen entsprechen.

2) Vgl. KenNeL, Arb. Zool. Instit. Würzburg, VI, 1853, p. 267. 
Die meisten der von mir erbeuteten Majoidea, Huenia grandidieri, Menaethius monoceros, Acanthonyx quadridentatus, Pseudomicimpe nodosa, Micippe philyra, Tylocarcimus styx, sind Bewohner von Korallriffen, scheinen aber nicht an Korallen, sondern an die dortigen Tang- und Seegraswiesen gebunden zu sein. Sie leben dort in gleicher Weise wie die Dromiiden, und ihre Bewegungen sind äusserst träge. Ihr Rücken ist stets mit kleinen Algen und anderen Seegewächsen besetzt, so dass sie leicht übersehen werden. Auch ihre Färbung ist durchweg ihrem Aufenthalt angepasst, meist im Leben ein trübes Grün. Bei Kilwa schleppte ich in 8-10 m Tiefe auf sandig-schlammigem Grunde, auf dem ebenfalls Seegras vorhanden war: Huenia grandidieri, Stenorhynchus brevis, I'aratymolus muescens, Hyasthenus brevicornis. Huenia protens lebt bei Tokio zwischen Algen ${ }^{1}$ ). Die genannten Formen werden demnach wohl alle, nebst vielen Verwandten, die Seegras- und Algen-Facies bevorzugen.

Den grössten Theil der Schwimmkrabben, Portınidae und Thalamitidae, erlangte ich im Hafen von Dar-es-Salaam, und zwar leben die kleineren Formen: Neptunus gramulatus, Goniosoma affine und danae, Thalamita savignyi, wie die jungen Exemplare von Neptumus sanguinolentus an eben den Stellen, wie Calappa und Mratuta, d. h. dicht am Strande in ganz flachem Wasser. wo sie in derselben Weise dicht über dem Sandgrund hinschwimmen und sich in demselben verbergen. Die grösseren Formen, Neptunus sanguinolentus ad., Scylla serrata, ziehen etwas tieferes Wasser vor. Thalamita crenata ist eine der häufigsten Formen und hält sich an Orten auf, die ihr gute Verstecke bieten, wo z. B. Baumstämme oder einzelne grössere Steine im Wasser liegen. Beunruhigt, setzt sie sich muthig zur Wehre, indem sie möglichst ihren Rücken zu decken sucht und nach vorgehaltenen Stöcken etc. mit beiden Scheeren kräftig kneipend zuschlägt.

Die Formen der Tanthini, Familie: Menippidae, Tunthidae, Oziidue, Trapeziidae, erreichen ihren grössten Formenreichthum in den tropischen Meeren, wo sich besonders auf den Korallenriffen zahlreiche Vertreter von ihnen finden. Nur wenige Stücke habe ich an anderen Localitäten erbeutet: ein Exemplar von Myomcnippe panope, Eurycarcinus natalensis, Epixanthus dentatus, und zwar fanden sich diese am äussersten Ende der südlichen Bucht des Hafens von Dar-es-Salaam auf sandig-schlammigem Grunde. Die auf Korallenriffen lebenden Formen zeigen zunächst eine Anzahl solcher, die lebende Korallen bewohnen, wo sie sich in dem Geflecht reichverzweigter Formen (Pocillopora, Ysammocora, Lophoseris u. a.) aufhalten. Als solche sind zu nennen: Cymo andrcossyi. Actaea cavipes, Thymodius ungulatus, Chlorodius niger, und besonders Trapezia cymodoce, rufopunctatu und Tetralia glaberrima. Die drei letzteren sind ganz besonders als Bewohner der lebenden liorallstöcke aufzuführen, und die ïbrigen Trapeziulae werden sich ihnen anschliessen. In Löchern und Höhlungen an der Basis lebender Korallen, sowie auch im todten Koralkalk kommen vor: Myomenipme panope (auch auf anderer Facies), Pseudozins caystrus, Pilumns vespertilio, Xantho exaratus (auch an felsigen Strand bei Kilwa), Actaea tomentosa, Actuea mfopunctata (diese Art wird in der Wahl der Facies wenig sich beschränken, da sie eine weite Verbreitung besitzt), Zozymus aeneus, Lophactaea cristatu, Hypocoelus sculptus, die Carpilodes-Arten, Carpilius convexus. Ozins mgnlosus, Epixanthus corrosus. Viele dieser Arten mögen nicht die Korallen, sondern überhaupt felsigen, höhlenreichen Grund bevorzugen. Eurïppellia ammlipes, Eriphia laevimana und smithi fehlen fast ganz auf dem lebenden Riff, finden sich dagegen auf dem dicht am Strande liegenden abgestorbenem Korallkalk. Besonders Eriphia smithi ist an solchen Stellen des Strandes, wo sich todter Korallkalk findet, in den Löchern des letzteren äusserst gemein. Zur Ebbezeit findet man sie oft ausserhalb des Wassers. Nach Art der Thalamita crenatı setzt sie sich bei Angriffen zur Wehre, indem sie mit beiden Scheeren gleichzeitig kräftig zuschlägt. Auffallend ist es, dass ich bei der Häufigkeit der E. smithi nur ein Exemplar von E. laevimana erbeutete.

Ueber das Vorkommen der einzelnen Grapsidae kann ich folgende Angaben machen. Die beiden Metopograpsus-Arten und den Heterograpsus erythraeus erbeutete ich in Hafen von Dar-es-Salaam, wo sie an dem Holzwerk der Landungsbrücken sich aufhalten. Grapsus grapsus ist ganz charakteristisch für die steilen, von der Brandung bespritzten Uferfelsen, wo er mit unglaublicher Gewandtheit und Schnelligkeit herumklettert. Ich sah ihn fast überall an derartigen Localitäten, konnte ihn aber nur schwer erbeuten. In Bezug auf Färbung und Lebhaftigkeit der Bewegungen gehört er zu den schönsten Krebsen. Grapsus

I) Vgl. Ortmann, Zool. Jahrb, VIl, I893, p. 40.

Jenaische Denkschriften. VIII.

S emon, Zoolog. Forschungsreisen. V. 
strigosus hält sich an ähnlichen Orten, besonders in und an den Strudellöchern am Ras Chokir auf, doch fand ich ihn auch im Hafen an Holzwerk. Seine Färbung ist in der Hauptsache trüb-grün. Varuna litterata, Plagusia immaculata und Leiolophus abbreviatus scheinen, nach dem, was über sie bekannt wurde, in gewvisser Beziehung pelagische Formen zu sein, die sich an schwimmenden Gegenständen aufhalten ${ }^{1}$ ).

Die Arten der Gattung Sesarma und Helice sind keine marinen Krebse. Ihr Aufenthaltsort scheint an Süsswasser gebunden zu sein. Die am Hafen von Dar-es-Salaam erbeuteten Exemplare halten sich dort am Fusse des steilen Uferabhanges auf, der überall kleine Süsswasserquellen aufweist, und wo sie sich oberhalb der Fluthgrenze ihre Löcher graben. Ich sah sie niemals im Seewasser selbst. Dagegen laufen sie vielfach auf dem Lande umher. Ses. meinerti kommt selbst weit von der See entfernt vor, z. B. im Upanga-Thal, zwei Kilometer von der Mündung des Flusses entfernt, in den (auch zur trockenen Jahreszeit) völlig von Süsswasser gebildeten Sümpfen. Sie gräbt sich ebenfalls tiefe Löcher und läuft, wie die anderen Arten, auf dem Trocknen umher. Am ganzen Aussenstrand von Dar-es-Salaam, wo Süsswasser fehlt, fand ich niemals eine Sesarma ${ }^{2}$ ). Die Scheeren von S. meinerti sind nicht zu verachtende Waffen, die sie auch geschickt und in empfindlicher Weise zu gebrauchen versteht.

Cardisoma carnifex lebt unter ähnlichen Bedingungen, wie Sesarma meinerti, und oft in deren Gesellschaft. Sie entfernt sich vielleicht noch weiter von der See und bewohnt trockenere Orte als diese, wo sie sich tiefe Löcher gräbt. Bei Dar-es-Salaam ist sie sehr häufig, jedoch sah ich während der trockenen Jahreszeit selten ein Exemplar dieser Art.

Dotilla fenestrata ist im Hafen von Dar-es-Salaam der gemeinste Krebs. Sie lebt am sandigen Strande innerhalb der Ebbezone zu Millionen. Zur Fluthzeit sieht man von ihr nichts, da sie in ihren zugeschwemmten Löchern sitzt, sobald aber bei Ebbe ihre Wohnplätze von Wasser frei werden, beginnt sie zunächst ihre Löcher zu säubern und dann auf Nahrung auszugehen, indem sie den feinen Sand durchkaut. Der durchgekaute Sand wird in Form kleiner Würstchen aus den Nundtheilen herausgepresst, und da sie immer ab und zu wieder in ihr Loch schlüpft und hervorkommt, um in einer anderen Richtung ihre Fressthätigkeit fortzusetzen, bilden sich auf dem glatten Sandboden ganz charakteristische sternförmige Figuren: in der Mitte ein Loch, von dem eine Anzahl, $10-20 \mathrm{~cm}$ langer Furchen ausstrahlen, deren jede auf einer Seite von einem Wall kleiner Würstchen begrenzt ist. Bei zurückkehrendem Wasser zieht sie sich vor demselben strandaufwärts zurück, so dass dicht am Rande des steigenden Wassers in der Breite von mehreren Metern der Sand mit zahllosen Exemplaren bedeckt ist, die oft nicht neben einander Platz haben, so dass sie in mehreren Schichten über einander herumlaufen. Nähert man sich diesem lebenden Strandwall, so beginnt die ganze Gesellschaft zu flüchten und sich in den Sand einzugraben, was den oberen aber erst gelingt, wenn die unteren unter dem Sande verschwunden sind. Das Geräusch, das ihr Laufen hervorbringt, gleicht dem eines starken Platzregens. Auch bei Kilwa lebt diese Art in grossen Colonien in ähnlicher Weise zusammen. Ihre blass-gelbliche oder blass-grünliche Farbe passt sich gut der Farbe des Sandes an.

Nach der oben gegebenen Notiz SEMon's scheint Myctiris longicarpus bei Amboina unter ähnlichen Verhältnissen zu leben.

Die vier von mir gesammelten Arten der Gattung Gelasimus sind sicher gut von einander geschieden, was schon daraus hervorgeht, dass sie niemals unter einander vorkommen. Selbst wo dieselben dicht bei einander gefunden werden, sucht sich jede ihre gesonderten Wohnplätze aus. Am besten konnte ich dies bei Lindi beobachten. Die rechte Seite des dortigen Flussufers wird von einem nicht sehr breiten Man-

I) Keine einzige Form der Grapsinae, noch weniger der Gattung Grapsus selbst gehört zu den Landkrebsen, Walther (Bionomie des Meeres, p. 95) führt Grabsus (sic!) unter den Landkrebsen auf; wem er diese Notiz entnommen hat, ist mir unbekannt, vielleicht F. MülLER, Für Darwin, 5. Capitel, aber auch hier spricht F. MüLler nur von einer nicht näher definirten Grapsus-Art, bei der er gelegentlich Luftathmung beobachtet haben will. Die Identification dieser Form ist aber völlig unmöglich. Die Unterfamilie der Sesarminae unterscheidet sich von den marinen (sehr selten süsswasserbewohnenden) Grapsinae durch gewisse Merkmale, die in Zusammenhang stehen mit den terrestrischen Gewohnheiten der ersteren. Ueber die Sesarminae vergl. F. MUller, 1. c., ferner Kingsley, Synopsis of the Grapsidae, Proceed. Acad. Nat. Sc. Philadelphia 1SSo, und DE MAN, Zool. Jahrb., II, I887, p. 64I. Hätte WaLTHER die citirten Stellen nachgesehen, so würde er wohl auf den Gedanken gekommen sein, dass ein irgendwo als Grapsus angeführter Landkrebs zu den Sesarminae zu rechnen ist.

2) Vgl. auch Bürger, Zool. Jahrb., VII, 1893, p. 613. 
grovengürtel begleitet. Unterhalb desselben, auf schlammigem Grunde, der mit Steinen und Geröll vermischt ist, findet sich G. cultrimamus, in den Mangroven selbst, in dem charakteristischen, zähen, schwarzen Schlamme findet sich G. urvillei, an der oberen Grenze der Mangroven, an reinen Sandstellen lebt G. anmulipes. G. inversus erbeutete ich mit diesem letzteren zusammen, doch machte ich damals beim Sammeln noch keinen Unterschied zwischen beiden Arten, so dass sie in ein Gefäss geriethen. Ich glaube aber bestimmt, dass auch von ihnen die einzelnen Colonien sich getrennt von einander halten. Bei Kilwa erbeutete ich nur zwei Arten: auch hier leben beide dicht benachbart mit einander, jedoch finden sich die Colonien von G. cultrimamus wieder auf Stellen, die ein Gemisch von Sand, Schlamm und Steinen zeigen, während G. ammutipes auf ganz reinem Sande auftritt. Bei Dar-es-Salaam leben ebenfalls alle vier Arten an den entsprechenden Stellen, nur nicht so dicht bei einander: G. urvillei findet sich erst am Südende der Hafenbucht im schwarzen Mangrovenschlamme. In der Lagune bei Zanzibar fand ich nur den G. annulipes, hier aber an Oertlichkeiten, wie sie sonst von G. cultrimanus bewohnt werden.

Die Arten der Gattung Gelasimus sind durchaus keine echten Landkrebse, sondern typische Strandbewohner, die sich nur innerhalb der Ebbezone aufhalten. Ihre Wohnplätze, auf denen sie in Schaaren zusammenleben, werden bei jeder Fluth vom Wasser bedeckt, und ich habe sie nur an solchen Stellen gefunden, bis zu denen das Seewasser reicht, wenn ich auch glaube, dass gewisse Arten (z. B. urvillei) häufig mit Brackwasser vorlieb nehmen müssen. Während der Fluth werden ihre Löcher zugeschwemmt, zur Ebbezeit beginnt ihre eigentliche Thätigkeit. Niemals habe ich gesehen, dass sie sich weit von ihren Löchern entfernen. Die eine enorm entwickelte, meist lebhaft gefärbte Scheere des $\delta$ dient keineswegs dazu, den Eingang zu den Löchern zu verschliessen (wie z. B. in BREHм's Thierleben zu lesen ist), sondern ist offenbar ein sexueller Zierrath, mit dem die $\delta$ die $q$ anlocken, und dieser Zweck wird noch dadurch gefördert, dass die $\delta$ die lebhaft gefärbte, hoch erhobene Scheere in eigenthümlicher Weise hin und her bewegen, was durch den Namen „Winkerkrabben" gut bezeichnet wird. Nur die Bewegung dieses auffallenden Anlockungsmittels erregt die Aufmerksamkeit der $q$, ruhende Gegenstände werden nicht beachtet. - An der Luft vermögen sie nicht lange zu leben, sobald sie aus ihrer feuchten Umgebung entfernt werden: die Exemplare, die ich in der Lagune ron Zanzibar fing und lebend mit mir nahm, waren nach einer halben Stunde alle abgestorben. Der Boden ihrer Wohnplätze ist aber stets feucht, und ich bin überzeugt, dass das Haarpolster, das sie unten zwischen den Basen des 3. und 4. Beinpaares tragen, keinen anderen Zweck hat, als die Bodenfeuchtigkeit gleich wie mit einem Schwamm aufzusaugen und den Kiemen zuzuführen.

Die Gattung Ocypode lebt ähnlich wie Gelasimus nur innerhalb der Ebbezone, und zwar an Strandstellen, die von reinem Dünensand gebildet werden, in welchem die Arten tiefe Löcher graben. Sie sind an reines Salzwasser gebunden und finden sich deshalb z. B. bei Dar-es-Salaam nur am Aussenstrande und Hafeneingang. Im Laufen leisten sie ganz Ausserordentliches, während sie ungeschickte Schwimmer sind und, ins Wasser getrieben, sich möglichst schnell in den Sand eingraben. "Ausschliessliche Landthiere" (wie in Brenn's Thierleben steht) sind sie durchaus nicht. Das Haarpolster zwischen dem 3. und 4. Beinpaar hat offenbar dieselbe Bedeutung, wie bei der Gattung Gelasimus. Ocypode ceratophthalma lässt, wenn sie in ihren Löchern sitzt, einen tief brummenden Ton hören, der offenbar von der auf der Innenseite der Hand befindlichen Stimmleiste durch Reibung gegen die Leiste des Ischium hervorgerufen wird. 


\section{Versuch einer Charakterisirung und Umgrenzung der Indo- pacifischen Region.}

Wenn ich im Folgenden den Versuch mache, die Indo-pacifische Region einer näheren Betrachtung zu unterziehen, so muss ich vorausschicken, dass ich speciell durch das vorliegende Material dazu veranlasst wurde, und deshalb auch mich völlig auf die Berücksichtigung der Decapoden-Krebse beschränke. Das Heranziehen anderer Thiergruppen erscheint theils deshalb nicht thunlich, weil dieselben vielfach anderen Verbreitungsgesetzen unterworfen sind, theils würde die Auswahl derselben schwer werden, da viele nur ungenügend bekannt sind.

Auch in Bezug auf die geographische Verbreitung der Decapoden weist unsere Kenntniss noch wesentliche Lücken auf. Doch halte ich das, was wir wissen, für genügend, um allgemeine Gesichtspunkte - wenigstens für die Indo-pacifische Region - aufzustellen. Durch neue Funde werden meinem Erachten nach wohl kaum die folgenden Ausführungen wesentlich abgeändert werden: dagegen ist zu erwarten, dass sie durch solche sich im Allgemeinen werden bestätigen lassen.

Die Indo-pacifische Region erstreckt sich, wie der Name sagt, über Theile des Indischen und Pacifischen Oceans, umfasst aber in ihrer charakteristischen Ausbildung nur das Litoral'1), d. h. den den Küsten unmittelbar anliegenden Theil der Oceane bis zu einer gewissen Tiefe. Man nimmt ziemlich willkürlich die Continentalstufe ${ }^{2}$ ), Io० Faden, als untere Grenze der Litoralregion an. Diese Grenze ist in Wirklichkeit nicht so scharf, aber ich halte es für den vorliegenden Zweck für überflüssig, auf diese Frage einzugehen. Jedenfalls sind von der Indo-pacifischen Region in ihrer typischen Ausbildung alle die Theile des Oceans ausgeschlossen, die als offenes Meer von Plankton und Nekton, oder als Tiefsee vom abyssalen Benthos und Nekton bewohnt werden: für diese gelten besondere Gesetze. Die Indo-pacifische Region um. fasst somit nur Theile des Litoralgebietes der Erde mit seinem Benthos, Nekton und Plankton. Unter den Decapoden ist besonders die erstere Gruppe, das Benthos, vertreten. An dieses echt litorale Benthos reihen sich Formen an, die die Schorre bewohnen, die Strandbewohner, und ferner fügen sich ihnen an, allerdings oft mit Modificationen in ihren Verbreitungsverhältnissen, eine Anzahl Süsswasser- und Landbewohner.

Einen allgemeinen Charakter der litoralen Indo-pacifischen Decapoden-Fauna kann man nicht mit ein paar Worten wiedergeben. Wesentlich ist ein ungeheurer Formenreichthum, die einzelnen Formen sind selten local beschränkt, sondern verbreiten sich meist über das ganze Gebiet. Von diesen Formen sind als charakteristisch vor allen diejenigen hervorzuheben, die in Folge ihrer Körpergrösse, ihrer Häufigkeit und anderer Ursachen auffallen, und der Indo-pacifischen Fauna an jeder beliebigen Stelle unter entsprechenden Verhältnissen ein besonderes Gepräge aufdrücken. Bisher wurde auf diesen Punkt wenig Aufmerksamkeit verwendet. Nach meinen Beobachtungen in Ost-Afrika giebt es aber eine ganze Reihe von Formen, die derartig ins Augen fallen, dass selbst der Laie auf sie aufmerksam wird.

1) Vgl. oben p. 3 Anmerk.

2) Vgl. Walther, Bionomie des Meeres, IS93, p. 4, II, 14. Die Definition der „Flachsee“, p. 14, ist zum mindesten unklar ausgedrückt. Wie will er eigentlich diesen Lebensbezirk begrenzen? Durch die roo-Fadenlinie oder durch die untere Grenze $(400 \mathrm{~m})$ des diaphanen Gebietes? 
Bei Dar-es-Salaam sind dies vor allen die Formen, die man am Strande auf Schritt und Tritt antrifft. Als solche nenne ich vor allen Dingen die Ocypoden, in den beiden Arten O. ceratophthalma und kuhli, die an sandigen Stellen des Strandes in Menge leben. Ihnen schliessen sich die an anderen Strandstellen vorkommenden Gelasimus-Arten an, besonders G. cultrimanus, die durch ihre Menge imponiren. Ferner sind die grossen Schwimmkrabben zu nennen, Neptunus sanguinolcntus, Scylla servata, die im Wasser selbst, auf sandigem Grunde, auffallen. Mit ihnen zusammen lebt Calappa hepatica und Matuta victrix. An felsigen Strandstellen fällt Eriphir smithi durch ihre Grösse und Wehrhaftigkeit, Calcinus horbsti durch seine Massenhaftigkeit auf. Das reichste Krebsleben entwickelt sich auf den Riften, wo sich gewisse Formen auch durch von ihnen hervorgebrachte Geräusche dem Ohre wahrnehmbar machen. Bei einem Gang über ein Riff wird man stets auf ein eigenthümliches knipsendes Schnalzen aufmerksam, das überall und ununterbrochen ertönt, ohne dass man die Urheber erblickt: beim Nachsuchen findet man, dass es von AlpheusArten (und Gonodactylus-Arten) herrührt, die in Unmengen im Korallkalk leben, und von denen viele für die Indo-pacifische Region charakteristisch sind. Von sonstigen Riffkrebsen fallen einige durch ihre Grösse auf, wie Prgurus punctulatus und Aeformis, Carpilius convexus, Zonymus aeneus, die übrigen entziehen sich meist dem Auge. Sehen wir uns unter den Landformen um, so treffen wir auch hier einige sehr auffallende Formen, wie die Coenoliten, Cardisoma carnifex, Sesarma meinerti.

Alle die genannten Formen, die bei Dar-es-Salaam so sehr auffallen, sind über das Indo-pacifische Gebiet ganz allgemein verbreitet, während einige andere bei Dar-es-Salaam zwar auch recht auftällig sind, aber entweder nicht alløemein an anderen Orten wiedergefunden werden oder mehr oder weniger kosmopolitisch sind (Dostilla fenestrata, Grapsus grapsus).

Fügt man noch die Formen hinzu, deren allgemeines Vorkommen, deren Häufigkeit und Körpergrösse vermuthen lässt, dass sie sich, entsprechend ihrer Verwandtschaft mit den genannten, ähnlich verhalten, so würden wir die folgende Liste von Charakterformen der Indo-pacifischen Decapoden-Fauna aufstellen können.

I. Rein marine Formen (nie ausserhalb des Wassers).

a) Riffbewohner, sowie Bewohner der bei Dar-es-Salaam an die Riffe gebundenen Tang- und Seegraswiesen.

Alpheus laevis, machrochirus und viele andere Arten. Pagurus punctulatus, deformis u. a. Arten, Zozymus ueneus, Carpilius convexus und maculatus.

b) Auf Sandgrund:

Calappa hepatica, Matuta victrix nebst ihren Varietäten. Neptunus pelagicus, sanguinolentus, Scylla serrata, Thalamita crenata und andere Arten.

II. Schorrenbewohner.

a) An felsigem Strand:

Calcinus herbsti, Eriphic laevimana und smithi.

b) Dünenbewolner, an Salzwasser gebunden:

Ocypode kuhli, ceratophthalma und andere Arten.

c) Brackwasserbewohner, meist wohl euryhalin.

Gelasimus cultrimanus, annulipes, urvillei und viele andere Arten.

III. Süsswasser- und Landformen.

Coenolita clypeatus, rugosus, compressus, perlatus, Birgus latro, Sesarma meinerti und andere Arten, Cardisoma carnifex.

Diese Liste macht durchaus keinen Anspruch auf Vollständigkeit; sie würde sich leicht um eine ganze Reihe von Arten vermehren lassen, besonders wenn man auch die weniger auffallenden und die in tieferem Wasser lebenden, die sich der directen Beobachtung entziehen, hinzuzieht. Die genannten Arten sind solche, bei denen sich aus den bisher bekannten Daten ihres Vorkommens schliessen lässt, dass sie sich wohl stets an jeder beliebigen Stelle des Indo-pacifischen Gebietes an den entsprechenden Localitäten werden nachweisen lassen. 
Die Grenzen der Indo-pacifischen Region lassen sich im Allgemeinen rein empirisch feststellen. Es ist eine Thatsache, dass die dieser Region angehörenden Decapoden meist eine ganz eigenthümliche und gemeinsane Verbreitung haben, die über gewisse Grenzen nicht hinausgeht. Formen, die sich anders verhalten, bilden nur Ausnahmen. Die Grenzen, bis zu denen die Hauptmasse dieser Formen vordringt, sind zum Theil sehr scharf und durch die geographischen Grenzen von Meer und Continent bedingt. So bildet im Norden des Indischen Oceans vom Rothen Meere bis Hinter-Indien der asiatische Continent die Nordgrenze für die Indo-pacifische Fauna, in Westen der afrikanische Continent. Die Grenzen nach Süden und Osten, sowie östlich von Asien nach Norden sind nicht durch vorliegende Landmassen bestimmt. In diesen Richtungen können wir dieselbe Fauna nördlich bis Japan, westwärts über die ganze pacifische Inselwelt, bis zu den Sandwich- und Paumotu-Inseln verfolgen. Im Süden begleitet die Indo-pacifische Fauna die Ost- und Westküste Australiens und erstreckt sich an der Ostküste Afrikas fast bis zum Cap (vgl. unten).

Aus der oben gegebenen Gruppirung der Charakterformen geht hervor, dass die Mehrzahl derselben sich an eine bestimmte Facies ${ }^{1}$ ) des Meeresgrundes bindet. Die genannten Formen der Facies sind nicht die einzigen, die im Litoral vorkonmen: es reihen sich an sie noch weitere an, die besonders tieferem Wasser angehören. Die Summe aller der Formen, die an die verschiedenen Facies des Litorals gebunden sind, bildet die Indo-pacifische Fauna in der Weise, dass diese Formen sich über ein bestimmtes Gebiet, wie es eben in grossen.Zügen begrenzt wurde, verbreiten, und dass sie sich innerhalb dieses Gebietes an jeder beliebigen Stelle finden können. Es ist leicht einzusehen, dass eine gewisse Continuität dieser Facies über das ganze Gebiet hin der wesentlichste Factor für dessen Gleichartigkeit ist. Wo irgend diese Facies auftreten, und wo sie in irgend welcher Verbindung stehen mit Localitäten, die Indo-pacifische Fauna zeigen, da ist dieselbe Fauna zu erwarten. Diese Continuität der Facies ist aber durchaus keine vollständige in räumlicher Beziehung, im Gegentheil, sie ist sehr häufig unterbrochen. Diese Unterbrechungen dürfen natürlich nicht so ausgedehnt sein, dass sie die Communication der Bewohner benachbarter Localitäten gleicher Facies verhindern: die Bewohner müssen über die Lücken hinübergelangen können. Das ist nun bei den Decapoden-Krebsen, die ja vielfach ein benthonisches Leben führen, für die erwachsenen Formen vielfach erschwert, aber in diesem Falle wird die Verbindung durch die pelagisch lebenden Larvenformen derselben ermöglicht. Im Indo-pacifischen Gebiet haben wir nun die Verbältnisse so liegend, dass wir zunächst eine ununterbrochene Küstenlinie von Süd-Afrika bis China haben, an der sich die verschiedenen Facies in fortwährender Wiederholung ablösen: dieser Wechsel ist so reich und tritt auf ganz kurze Strecken immer wieder auf, oft gehen die einzelnen Facies neben einander her, dass auf dieser Küstenlinie nirgends eine erhebliche Schwierigkeit der Verbreitung entgegentritt. Ferner aber sind einerseits die Inselgruppen des Indischen Oceans, andererseits die übrigen Gebiete der Indo-pacifischen Fauna östlich von Asien, unter sich und von dieser Küstenlinie durch so geringe Entfernungen getrennt, dass auch hier ein wesentliches Hinderniss für die Verbreitung der Decapoden-Krebse nirgends vorhanden ist ${ }^{2}$ ).

Sehr charakteristisch für das Indo-pacifische Litoralgebiet sind die Korallriffe. Da viele Decapoden an diese Facies gebunden sind und diese Form des Meeresgrundes auch gewisse andere Facies bedingt, so könnte man versucht sein, die Grenze des Indo-pacifischen Gebietes nach der Verbreitung der Korallriffe zu bestimmen ${ }^{3}$ ). Es würde das aber durchaus nicht correct sein, da die riffbewohnende Krebsfauna zwar einen beträchtlichen Theil der Indo-pacifischen Fauna ausmacht, aber neben ihr noch zahlreiche echt Indo-pacifische Elemente existiren, die nicht an Korallen gebunden sind und ihre Verbreitung weiter auf angrenzende Litoralgebiete ausdehnen, in denen Riffkorallen fehlen: die Riffkorallen haben eben andere

1) Vgl. Walther, Bionomie des Meeres, p. $25 \mathrm{ff}$.

2) Ueber die Verbreitung der Larvenformen litoraler Krebse und ihren weiten Transport durch Meeresströme etc. vgl. Ortmann, Decapoden und Schizopoden der Plankton-Expedition, I893, p. 97-99, p. 105-110 u. pl. 10. - Daselbst ist zu ersehen, dass die Entfernungen, bis zu denen der Transport solcher Larven erfolgt, vielfach viel bedentender sind, als irgend eine Entfernung zwischen beliebigen benachbarten Punkten des Indo-pacifischen Litoralgebietes. - Vgl. auch STUDER, Gazelle, III, I889, p. 32.

3) Vgl. Walther, Bionomie des Neeres, p. 3o. Walther identificirt die "Indo-pacifische Provinz" geradezu mit der F a cies der Riffkorallen. Er vergisst dabei, dass auch in West-Indien Korallriffe vorkommen. Interessant ist die Vergleichung einer Provinz mit einer Facies. 
Verbreitungsgesetze ${ }^{1}$ ). An gewissen Stellen fallen aber die Grenzen des Verbreitungsgebietes der Riffkorallen und der litoralen Decapoden-Krebse zusammen: Zunächst im Osten des Gebietes, wo der offene Pacifische Ocean beiden Thierklassen eine Schranke entgegensetzt, die sie nicht überwinden können. Die grossen Tiefen machen ein Weitervordringen der benthonischen Litoralformen unmöglich, und ebenso scheinen für die planktonischen Larven die weiten Wasserflächen mit ihren verschiedenen Verbreitungsmitteln nicht günstig zu sein für eine weitere Ausbreitung nach Osten. Dieselben Verhältnisse treten auf in der Südrichtung, zwischen Afrika und Australien und östlich von Australien, wo die Tiefsee und die weiten Wasserflächen des südlichen Indischen Oceans und des südlichen Pacific gleichfalls Grenzen ziehen.

Wir hätten somit die topog raphischen Ursachen der Umgrenzung des Indo-pacifischen Gebietes nach den hauptsächlichen Richtungen hin festgestellt. Nach IVesten bildet das Festland von Afrika, nach Norden zu einem grossen Theil das Festland von Asien, nach Osten und zum Theil nach Süden der offene Pacific und freie südliche Indische Ocean ein Verbreitungsh indern iss für die litoralen Indo-pacifischen Decapoden. Innerhalb dieser Grenzen haben wir durchweg eine Continuität der Facies, die nur durch kleine Lücken unterbrochen wird, die sich durch die Verbreitungsmittel der Decapoden überwinden lassen, während an den genannten Grenzen weite und unübersteigbare Schranken dadurch gebildet werden, dass die Facies sich durchgreifend und auf grössere Strecken hin ändern. Das eine Mal tritt an die Stelle der marinen Litoral-Facies, wenn man so sagen will, eine continentale Faciesgruppe, das andere Mal werden die ersteren von denen der Tiefsee und durch den offenen Ocean abgelöst.

Die litoralen Facies, die im Indo-pacifischen Gebiete beobachtet werden (mit Ausnahme der RiffFacies), setzen sich aber an drei Stellen noch weiter mit einer ähnlichen Continuität wie sonst in Gegenden fort, die nach unserem empirischen Material nicht mehr die Indo-pacifische Fauna zeigen, nämlich an der südlichen ost-afrikanischen Küste, gegen das Cap zu, an der ost-asiatischen Küste über Japan in den nördlichen Pacific und drittens an der Ost- und Westküste Australiens nach Süden zu. Untersuchen wir diese drei Stellen genauer, und versuchen wir festzustellen, wie weit sich empirisch die Indo-pacifische Fauna hier über das Gebiet der Riffkorallen hinaus erstreckt, und welchen Ursachen ihre Begrenzung zuzuschreiben sei.

Was den südlichen Theil von Ost-Afrika anbetrifft, so liegt uns von Specialarbeiten besonders ein Aufsatz von KraUss ${ }^{2}$ ) vor, und ferner finden sich auch sonst in der Literatur zerstreute Angaben über die Cap-Fauna. Das eigentliche Cap selbst (Tafelbai, Simonsbai) besitzt faunistische Elemente, die ihm eigenthümlich sind, die im Indo-pacifischen Gebiet fehlen, und andererseits sind echte Indo-pacifische Formen ebenda kaum vorhanden. Zur eigenthümlichen Cap-Fauna gehören z. B.: Pseudodromia latcns, Mursia cristata, Hymenosoma orliculare, Acanthonyx dentatus, Portumnus pulchellus, während andere sich z. B. in SüdAmerika und Süd-Australien wiederfinden, wie Iasus lalandei, Mlatyonychus bipustulatus, Cyclograpsus punctatus (die beiden letzteren mit Vorstössen in die Indo-pacifische Region).

Die Natalfauna (nach KRAUss) besitzt von diesen echten Cap-Formen, soweit sie bekannt wurden, nur den Acanthonyx dentatus. Dagegen treten eine Reihe echt Indo-pacifischer Formen auf, wie z. B.: Calcimus herbsti, MIatuto victrix, Neptunus pelagicus, Thalamita admete, prymna, crenata, Scylla serrata, Atergatis floridus, Xantho exaratus, Eriphia smithi u. a. Da ferner bei Natal noch Korallriffe vorkommen, so haben wir die Natal-Fauna als einen Theil der Indo-pacifischen anzusehen.

Die vorliegende Sammlung von Port Elisabeth, einer noch weiter südlich liegenden Localität als Natal, enthält nun schon folgende echte Cap-Formen: Psendodromia latens, Mursia cristata, Hymenosoma orliculare, Acanthonyx dentatus, Portumnus pulchellus, Platyonychus bipustulatus, Cyclograpsus punctatus, während als Indo-pacifische Arten nur: Lophozozymus dodone, Eriphia smithi, Ocypode kuhli und ceratophthalma anzusprechen sind. Hier in der Nähe muss also die Südgrenze der Indo-pacifischen Region liegen: wie sich die zwischen Natal undPort Elisabeth liegenden Küstenstriche verhalten, ist leider unbekannt. Die Strecke, auf der der Uebergang der beiden Faunen stattfindet, ist eine verhältnissmässig ksurze ${ }^{3}$. Auf alle Fälle ist aber

1) Vgl DANA, Corals and Coral Islands.

2) KiRauss, Südafrikanische Crustaceen, 1843 .

3) Jedenfalls reicht die Indo-pacifische Fauna bis nahe an Port Elisabeth heran, einzelne Elemente gehen selbst bis dorthin Walther (Bionomie des Meeres, p. S2) hält es, wie sein Gewährsmann Buckland, für ein ausserordentliches Ereigniss, dass bei 
als empirische Thatsache festzuhalten, dass die Indo-pacifische Fauna südlich sich nicht bis zur Südspitze Afrikas erstreckt ${ }^{1}$ ). An dieser Stelle liegt also ein Verbreitungshemmniss, das die Communication mit dem Atlantischen Litoralgebiet an der Westseite Afrikas verhindert. Eine topographische Ursache, ein plötzlicher Wechsel der Facies liegt hier nicht vor, der Grund ist in anderen Verhältnissen zu suchen. (Vgl. unten.)

Untersuchen wir nummehr die empirischen Grundlagen, nach denen wir die Grenze der Indopacifischen Region an der Ostseite Asiens nach Norden feststellen können. Dieselbe ist jedenfalls in der japanischen Inselgruppe zu suchen. Ueber die japanische Fauna liegt uns zunächst das grosse Werk DE HAAN's vor, das jedoch in Bezug auf diese Frage wenig Anhaltspunkte giebt, da die japanischen Fundorte nicht genauer angeführt sind, und gerade die genaue Angabe der Localität hier von grösster Wichtigkeit ist. Abgesehen von den in anderen Werken ${ }^{2}$ ) vorliegenden detaillirten Angaben, bin ich in der Lage, in diesem Gebiete genauere Angaben zu machen, da mir die reichen Sammlungen vorliegen, die Herr Professor Döderlein in Japan zusammenbrachte, und die ich zum Theil schon publicirt habe ${ }^{3}$ ). Diese Sammlungen sind deshalb besonders interessant, weil sie an verschiedenen Punkten Japans gemacht wurden, von den Liu-kiu-Inseln bis Tokio ${ }^{4}$ ), also sich über eine grosse Strecke des japanischen Inselreiches erstrecken. Eine ganze Reihe von Krebsformen ist für dieses Gebiet charakteristisch, jedoch ist zu bemerken, dass die Zahl dieser Arten seit DE HAAN's Zeiten fast stetig abnimmt, da der Nachweis geführt wird, dass sie im Indo-pacifischen Gebiet weiter verbreitet sind.

Die Liu-Kiu-Inseln sind rein Indo-pacifisch, was schon aus dem Vorhandensein von Korallriffen daselbst hervorgeht. Die Riffe verschwinden dicht vor der Südspitze des eigentlichen Japan. Ebenda liegt ein Ort, an dem Herr Professor DöDERLEıN sammelte, Kagoshima.' Unter dem von dort stammenden Material befinden sich folgende echt Indo-Pacifischen Formen ${ }^{5}$ ):

$\begin{array}{cl}\text { Alpheus crinitus, } & \text { Petrolisthes japonicus, } \\ \text { " parvirostris, } & \text { Pagurus sculptipes, } \\ " \quad \text { collumanus, } & \text { Dorimpe dorsipes, } \\ \text { " laevis, } & \text { Oreophorus rugosus, } \\ " \quad \text { pachychims, } & \text { Arcania undecimspinosa, } \\ \text { Hippolyte (Saron) giblerosa, } & \text { Myra fugax, } \\ \text { Coralliocuris superbu, } & \text { Paratymolus pubescens, }\end{array}$

Oncinopus aranca,

Hyastenus diacanthus,

Thalamita sima.

Actaea cavipes,

Actumus setifer.

Eine Anzahl hiervon haben wir oben als Korallenbewohner kennen gelernt: es sind also entweder bei Kagoshima Korallriffe in der Nähe vorhanden, was sehr gut möglich sein kann, oder die betreffenden Formen: Alpheus laevis etc., Saron gibberosus (affin. marmoratus, vergl. oben p. I3), Coralliocaris superba, Actaea cavipes sind nicht streng an Korallen gebunden.

Ausser diesen echten Indo-pacifischen Formen treten aber bei Kagoshima eigenthümliche Formen auf, die theils für Japan bezeichnend sind, theils sonst nur über die Liu-Kiu-Inseln und China sich verbreiten: es mag in der in Rede stehenden Sammlung ihre Zahl etwa ein Dutzend betragen.

Betrachten wir die Döperlein'sche Sammlung von Tokio, dem nördlichsten Fundpunkte, der in diesem Material vertreten ist, so hat sich die bei Kagoshima vorhandene Zusammensetzung nicht wesentlich verändert. Bei Tokio wurde, da dort längere Zeit gesammelt wurde, ein viel reicheres Material erbeutet,

Port Elisabeth im Jahre 1887 "4 Stïck von Pelamis bicolor, einer giftigen Schlange von Sum a tra" (!) ans Land getrieben wurden. Dem gegenüber ist zu bemerken, dass Pelamis bicolor die gemeinste Seeschlange ist, die von Madagascar bis Panama vorkommt, häufig auf hoher See beobachtet und häufig verschlagen wird.

1) Vgl. auch STuner, Gazelle, III, 1889, p. 51 .

2) Besonders Stimpson, Proceed. Acad. Nat. Sc. Philadelphia, 1857-60, und die Challenger-IVerke: Mers, Brachyura, 1886, Bate, Macrura, 1888, Henderson, Anomura, 1885.

3) Zool. Jahrb., V, ISgo, - VII, 1893.

4) Vgl. die Localitätsangaben bei ORTMANN, Japanische Cephalopoden, Zool. Jahrb., III, ISSS.

5) Die Liste ist nicht vollständig. Icl nenne nur echte Litoralformen und davon nur solche, deren Verbreitung eine grössere ist, oder die sonst charakteristisch sind. 
und deshalb enthält die hier folgende Liste mehr Arten, als die von Kagoshima. Viele der in letzterer felılenden werden aber wohl nur durch Zufall dem Sammler entgangen sein.

Bei Tokio wurden folgende echt Indo-pacifischen Formen erhalten:

\begin{tabular}{|c|c|}
\hline Penaens canaliculatus, & Arcania undecimspinosa, \\
\hline semisulcatus, & Myra fugax, \\
\hline Alpheus laevis, & Huenia proteus, \\
\hline Panulirus longipes (var. japonica), & Hyastemus diacanthus, \\
\hline Petrolisthes japonicus, & Schizophrys aspera, \\
\hline Pagurus sculptipes, & Micippe thatia, \\
\hline Aniculus aniculus, & Carupa laeviuscula, \\
\hline Dromia rumpli, & Neptunus gladiator, \\
\hline Dorippe dorsipes, & hastatoides, \\
\hline Matuta victrix, & pelagicus, \\
\hline
\end{tabular}

Neptunus sanguinolentus,
Goniosoma ornatum,
Thalamita sima,
$\quad$ ", mryma,
Cryptopodia fornicata,
Xantho exaratus,
Atergatis floridus,
$\quad$ "integerrimus,
Actumnus setifer,
Ocypode ceratophthalma.

Auch diese Liste macht keinen Anspruch auf Vollständigkeit. Die von mir in Ost-Afrika an Korallen beobachteten Formen treten ganz zurück (nur Alpheus laevis ist hier noch vorhanden, der demnach sicher nicht auf die Korallenfacies beschränkt ist), dagegen wurde von einer Anzahl ihr Vorkommen auf anderer Facies innerhalb der eigentlichen Tropen nachgewiesen. Auch bei Tolio tritt zu diesen Elementen des Indo-Pacific eine grössere Anzahl (mindestens 70) eigenthümlicher Formen, die auf das japanische Gebiet und die benachbarten Gegenden beschränlst sind, während wieder andere weitere Beziehungen erkennen lassen.

Zu den letzteren gehören zunächst solche Formen, die sowohl in Japan als auch im Nord-Pacific und auf der IVestseite Nord-Amerikas (bis Californien) vorkommen. Ich nenne folgende:

Eupagums samuelis.

Pugettia qualividens (und var. gracilis).

Hyastenus japonicus und longipes (die identisch sein sollen).

Cheiragonus cheiragonus.

Pachygrapsus crassipes.

In diese Kategorie ist auch das Vorkommen von Lithodes- und Cuncer-Arten in Japan, Nord.Pacific und an der Westküste Amerikas zu rechnen.

Ferner finden sich in Japan circumpolare Arten, wie z. B. Crangon crangon.

Ganz merkwürdiger Weise sind einzelne Arten Japan und dem Mediterrangebiete gemeinsam, wie Nika edulis, Pagurus striatus; jedoch sind diese beiden die einzigen mir bekannten Beispiele. Von anderen Arten, die unter diese Kategorie früher gerechnet wurden, sind neuerdings weitere Fundpunkte bekannt geworden, so dass ihre Verbreitung eine weitere zu sein scheint.

Selbstverständlich fehlen in Japan auch kosmopolitische oder nahezu kosmopolitische Formen nicht.

Die Crustaceen-Fauna Japans, nördlich bis Tokio, setzt sich demnach wesentlich zusammen aus eigenthümlichen Formen und aus echten Indo-pacifischen Elementen. Andere Beimischungen treten zurück. Aus diesen Thatsachen lässt sich der empirische Satz aufstellen, dass die japanischen Inseln bis Tokio eine Indo-pacifische Fauna zeigen, die eine reich entwickelte locale Ausbildung erlangt hat. Sie bilden ein ziemlich selbständiges Grenzgebiet, das sich aber von der Indo-pacifischen Region nicht als gleichwerthige Region abtrennen lässt. Bei Tokio ist eine scharfe Nordgrenze der Indo-pacifischen Region noch nicht erreicht.

Die Fauna des Nord-Pacific ist aber il ihrer speciellen Zusammensetzung noch wenig beliannt, und ebenso fehlen uns reichhaltigere Sammlungen aus dem nördlichen Japan, so dass man die empirische Nordgrenze der Indo-pacifischen Region in diesem Gebiete noch nicht sicher bestimmen kann. So viel steht aber fest, dass sich die Indo-pacifische Fauna nicht in den nördlichen Pacific fortsetzt und noch weniger die Westküste von Nord-Amerika erreicht, die einen ganz anderen Charakter aufweist. 
An der dritten Stelle, an der die Indo-pacifische Region nicht durch topographische Grenzen abgeschlossen wird, an der Ost- und Westseite Australiens lassen uns die vorliegenden Nachrichten fast ganz im Stich, da gerade von den interessanten Punkten an (Sydney und Shark-Bay) in südlicher Richtung detaillirte Angaben fehlen. Sydney auf der Ostküste ${ }^{2}$ ) und Shark-Bay auf der Westküste ${ }^{2}$ ) scheinen noch rein Indo-pacifische Fauna zu besitzen. Jedoch treten dann in Süd-Australien und Tasmanien andere Elemente auf ${ }^{3}$ ), so dass wir hier vielleicht ein analoges Verhalten haben, wie in Süd-Afrika.

Es ist also höchst wahrscheinlich, dass an den besprochenen drei Localitäten die Indo-pacifische Region entweder nach Süden oder Norden abgegrenzt wird: die Ursachen dieser Begrenzung sind nicht in topographischen Verhältnissen zu suchen. Es liegt nichts näher, als hier das Klima mit seinen Wirkungen heranzuziehen, und jedenfalls dürfte es nicht ïbereilt sein, der Abnahme der Meerestemperatur die wesentlichste Rolle zuzuschreiben. Es würden also hier klimatische Grenzen vorliegen, und die nach den klimatischen Verhältnissen des Litorals an besagten Stellen th eoretisch gezogenen Grenzen dürften wohl mit den empirisch festzustellenden zusammenfallen.

Betrachten wir auf der Karte der Meeresströmungen von KRÜMıEL (Oceanographie, II, I887) die Verhältnisse in Süd-Afrika, so sehen wir, dass an der Stelle, wo sich die ost-afrikanische Kïiste schärfer nach Westen umbiegt (bei der Algoa-Bai und Port Elisabeth), der Agulhas-Strom, der von Norden warmes Wasser nahe der Küste südwärts und südwestswärts führt, sich von der Küste, vom Gebiete des Litorals entfernt, und dass an dieser Stelle sich kaltes Wasser, von der Westwind-Trift herrührend, dicht an der Kiiste nach Osten und Norden vorschiebt. Nach den neuesten Untersuchungen von ScHotT ${ }^{4}$ ) liegen die Verhältnisse etwas complicirter. Schотт fand nahe der süd-afrikanischen Küste von Port Natal an bis fast zur Capstadt warmes, nach Süden und Westen strömendes Wasser, das jedoch südlich von Port Natal starke Schwankungen der Temperatur (zwischen $20,7^{\circ}$ und $14, I^{\circ}$ ) zeigte, erst von $2 I^{0} \mathrm{O}$. L. an, also nahe bei Capstadt, wurde diese Strömung verlassen und gleichmässig kaltes Wasser getroffen. Mag nun die Agulhas-Strömung näher oder weiter vom Lande verlaufen, jedenfalls constatirte auch ScHotT auf der in Frage kommenden Strecke, also jedenfalls von der Algoa-Bai an, Streifen von kaltem Wasser, bis nahe der Capstadt das IVasser durchweg kalt wurde. Gleiches kaltes IVasser, von der süd-atlantischen WestwindTrift aus höheren Breiten hergeführt, dringt als Benguela-Strom der Westküste Afrikas entlang aequatorwärts vor ${ }^{5}$ ). Durch diese Verhältnisse tritt eine starke Abkühlung der Gewässer an der Südspitze Afrikas und an der Westküste weit gegen den Aequator hin ein ${ }^{6}$ ), so dass an der ununterbrochenen Küstenlinie von der Algoa-Bai an, um das Cap herum, bis ziemlich zur Congo-Mündung eine breite klimatische Schranke von kaltem Küstenwasser liegt, das ansserdem, im Gegensatz zu den ziemlich gleichmässigen Temperaturen der tropischen Küstenwässer an der Ostseite Afrikas, starke Temperaturschwankungen zeigt. Diese breite Zone bildet offenbar das Haupthinderniss für die Verbreitung der Indo-pacifischen Litoralfauna um das Cap herum, und die Südgrenze derselben ist gemäss diesen Verhältnissen da zu ziehen, wo die Einwirkung der kalten Wasserstreifen beginnt, nach unseren bisherigen Kenntnissen etwa in der Gegend der Algoa-Bai.

Aehnlich scheinen die Verhältnisse in Japan zu liegen. Wenn sich hier in Folge von bestimmten topographischen Verhältnissen, auf die ich unten zurückkommen werde, an der Nordgrenze des Indo-pacifischen Gebietes eine besondere, reich entwickelte japanische Fauna herausbilden konnte, so wird schon dadurch ein mehr altmählicher Uebergang von der tropisch Indo-pacifischen Fauna zu der des Nord-paci-

I) Vgl. Hess, Decapoden-Krebse Ost-Australiens, IS65. Haswell, Catalog. Austral. Crust., 1882.

2) Vgl. die vereinzelten Angaben bei Mrers, Ann. Mag. N. H., (5) IV, I879, und Rep. Zool. Coll Alert, 18S4, ferner StUder, Gazelle, III, IS89, p $182 \mathrm{ff}$. - Die Fauna des Mermaid-Str. und des Dampier-Arch. ist echt Indo-pacifisch (ibid. p. I93).

3) Vgl. Pfeffer, Nied. Thierwelt d. antarct. Ufergebietes, Internat. Polarforsch. Die deutsche Exped., II, 1890, p. 462.

4) Wiss. Erg. Forschungsreise zur See etc., Peterdians's Mittheilungen, Ergänzungsheft, Io9, i893, p. 56 ff., fig. 5 auf. p. 57 und pl. 6.

5) Krünate, Oceanographie, II, p. 400.

6) Ausserdem wirkt daselbst ein kalter Auftrieb mit, vgl. KRÜMMEL, ibid. p. 308. 
fischen Litoralgebietes hergestellt. Wie wir aber gesehen haben, ist die japanische Fauna bis Tokio noch mit vielen echten Indo-pacifischen Elementen durchsetzt. Es liegt nichts näher, als dass man dies Verhältniss auf die Wirkung des durch den Kuro-Siwo-Strom nach Norden geführten warmen Wassers zurückführt. Doch liegt die Sache hier wesentlich complicirter, da z. B. der eigentliche Kuro-Siwo in einigem Abstande von der Ostliüste Japans verläuft, während das Küstenwasser selbst kälter ist ${ }^{1}$ ). In nördlichen Japan macht dann offenbar die von Norden kommende kalte Strömung ihren Einfluss geltend, und die Begrenzung des Indo-pacifischen Litorals wird wohl wesentlich von der Wirkung der kalten oder warmen Strömungen auf die Küstenwässer abhängig sein. Im Japanischen Neer, wo an der Ost- und Westseite verschieden gerichtete und verschieden temperirte Strömungen herrschen, werden jedenfalls besondere locale Unregelmässigkeiten zu erwarten sein.

Was schliesslich die australischen Verhältnisse anbetrifft, so giebt die KRÜMneL'sche Karte der Heerestrümungen im Wesentlichen an, dass sowohl an der West- als auch besonders an der Ostküste (südlich des IVendekreises) warme, nach Süden gerichtete Strömungen vorhanden sind, die eine Ausbreitung der Indo-pacifischen Fauna nach Süden begünstigen, während die ganze Südküste des australischen Festlandes unter der Herrsclaft der kalten Vestwind-Trift steht. Wie sich hier die Verhältnisse im Einzelnen gestalten, darüber fehlen die Beobachtungen: nach der Temperatur der Strömungen zu urtheilen, müsste aber an der Südküste Australiens, aber auch nur da, eine litorale Fauna sich finden, die von der Indopacifischen der Ostküste abweicht. Ob an der VVestküste verwickeltere Verhältnisse vorliegen, ist ungewiss: eigentlich sollte man dort kaltes Auftriebwasser erwarten, während, z. Th. wenigstens, das Gegentheil nachgewiesen ist $\%$.

Eine einheitliche Indo-pacifische Fauna existirt also in den oben des Näheren angegebenen Grenzen. In dieser Region finden sich im Wesentlichen ähnliche Bedingungen, die in einer gewissen Continuität stehen. Die verschiedenen Facies des Litorals wechseln oft und auf kurze Strecken, Orte gleicher Facies liegen aber stets verhältnissmässig nahe bei einander, so dass die Zwischenräume für die Decapodenkrebse keine Verbreitungshemmnisse bilden. Ebenso sind die klimatischen Verhältnisse ziemlich gleichmässig: die Region umfasst durchweg tropische oder subtropische Gegenden. Erst dort, wo sich die topographischen und kilimatischen Verhältnisse durchgreifend ändern und der Verbreitung der Fauna grosse Hindernisse entgegentreten, findet diese Region ihre Grenzen.

Nichtsdestoweniger zeigt die Indo-pacifische Litoralregion zu allen übrigen Theilen des Litoralgebietes der Erde gewisse Beziehungen. Am geringsten sind diese Beziehungen wohl zu den Gegenden, die ein nördliches Klima haben, zu den circumpolaren Litoralgebieten der nördlichen und südlichen Halbkugel. Wir haben schon oben gesehen, dass die Westlüste Nord-Amerikas zu Japan gewisse Beziehungen zeigt, und ebenso finden wir in tropischen Theil der Westliüste Amerikas einzehne, aber auch nur einzelne Formen, die im tropischen Indo-Pacific vorkommen, z. B.: Remipes testudinarius, Trapezia cymodoce und mfopunctata; die beiden letzteren Arten sind besonders deshalb erwähnenswerth, weil es die einzigen echten Korallbewohner sind, die die Schranke des offenen Pacific überwunden haben. Auch die Westlüste SüdAmerikas (Chile) besitzt in Grapsus strigosus, Leptograpsus variegatus u. a. Arten mit dem Indo-Pacific gemeinsame Züge. Diese letzten Formen konnten also die topographische Schranke der offenen See überwinden, während bei den japanisch-californischen Formen die Möglichkeit einer topographischen Verbindung im Nord-Pacific vorliegt: in diesem Falle musste aber die dort liegende klimatische Schranke überwindbar sein. Ferner ist bekannt, dass das Indo-pacifische Gebiet mit dem Atlantic gewisse Formen gemein hat, z. B. Alphens eldwardsi, Actaea rufopunctata, Grapsus grapsus, Pachygrapsus transversus. Für diese ist durchweg ein kosmopolitisches oder nahezu kosmopolitisches (circumtropisches) Vorkommen nachgewiesen. Alle diese Arten sind jedenfalls sehr eurytherm und vermögen die klimatischen Schranken am Cap, vielleicht auch im Nord-Pacific und an der Südspitze Amerikas zu überwinden. Jedoch muss ich 
darauf aufmerksam zu machen, dass Formen mit einer derartigen Verbreitung, deren es noch mehr giebt, die weniger kosmopolitisch sind, vielleicht als Relicte früherer geologischer Epochen anzusehen sind.

Werfen wir nunmehr einen Blick auf das gegenseitige Verhältniss der litoralen Regionen der Erde, sowie auf die Entstehung derselben. Es mag ein derartiger Versuch als verfrüht erscheinen, da unsere Kenntniss der Litoralfauna an vielen Punkten der Erde noch sehr lückenhaft ist: jedoch berufe ich mich darauf, dass schon anderweitig die Principien der marinen Thiergeographie behandelt worden sind. PFEFfER ${ }^{1}$ ) hat mit grossem Nachdruck darauf hingewiesen, dass es besonders zwei Factoren sind, die die jetzigen Verhältnisse herbeigeführt haben, und zwar ein klimatolog ischer und ein topog raphischer, und ich habe im Vorangehenden ebenfalls diese beiden Factoren, die sich vielfach entgegenarbeiten, in ausgedehnter Weise herangezogen. Ich würde mich den Ausführungen PFEFfER's völlig anschliessen können, wenn ich nicht in Bezug auf die Auffassung der arktischen und antarktischen Fauna anderer Ansicht wäre. PFEFFER geht von der Ansicht aus, dass die Fauna des antarktisch-notialen Ufergebietes mit der des arktisch-borealen viel grössere Aehnlichkeit besitze, als mit irgend einer dazwischen liegenden Fauna, und sucht diesen Umstand dadurch zu erklären, dass er beide aus einer gemeinsamen (vortertiären), über die ganze Erde verbreiteten Litoralfauna ableitet. Im Tertiär ging der allgemeine Charakter der alten Fauna verloren in Folge der Bildung von klimatischen Unterschieden, und zwar bildeten sich circumpolare Faunengebiete, indem sich in den höheren Breiten (zunächst an den Polen) von der allgemeinen Fauna besondere abgliederten, die sich an die abnehmende Temperatur anpassten. Bis hierher stimme ich vollkommen mit den Ausführungen PFefFer's überein. Weiterhin sucht er aber die „auffallende" Aehnlichkeit der Faunen um den Nord- und Südpol so zu erklären, dass die Fähigkeit, kaltes Wasser zu ertragen „bei den betreffenden Formen schon latent vorhanden war, ehe sie dazu kam sich zu bethätigen“, so dass nur bestimmte Gruppen sich an die Abkühlung gewöhnen konnten, ferner, dass „die allmähliche Abkühlung der polaren Gegenden und eine grössere Einförmigkeit der Lebensbedingungen hemmend auf die Umbildungsfähigkeit der organischen Substanz gewirlst hat", so dass sich "beide Faunengebiete in den erheblich langen Zeitläufen seit der Faunentrennung ausserordentlich wenig verändert haben". Mir ist der letztere Gedankengang völlig unverständlich. Nachdem bestimmte Gruppen sich an eine Abkühlung gewöhnt hatten, soll ihre Umbildungsfähigkeit abgenommen haben, so dass sie sich weiter nicht oder nur wenig veränderten: das kann man nur so verstehen, dass am Pole sofort, nachdem überhaupt eine klimatische Faunentrennung eintrat, ungefähr dieselben kalten Verhältnisse eintraten, wie sie jetzt dort herrschen. Alsdann konnten die angepassten Formen unter gleichbleibenden klimatischen Verhältnissen sich bis zur Jetztzeit annähernd unverändert erhalten. In dieser Weise hat man sich aber die Aenderung des Klimas nahe den Polen unter keinen Umständen vorzustellen, sondern die Abnahme der Temperatur wird eine allmähliche gewesen sein von tropischer bis zu arktisch kalter. Die Formen, die sich an die erste geringe Abnahme der tropischen Temperatur anpassten, mussten weiterhin sich an immer kühler werdende Temperatur gewöhnen, ihre Umbildungsfähigkeit musste im Gegentheil zunehmen, da ein Stillstand nicht eintrat, und durch die fortschreitende Ablühllung immer von neuem unter den zuerst angepassten Formen eine Auslese getroffen wurde. Dieselben wurden so zu sagen immer weiter decimirt, so dass nur ein kleiner Rest von solchen übrig blieb, die sich durch eine besondere Fähigkeit, kaltes Wasser zu ertragen, auszeichneten und sich dementsprechend verändert hatten. Dieser Vorgang ging unabhängig vor sich auf beiden Erdhälften, und wenn wirklich nahe dem Nord- und Südpol identische (?) oder auffallend ähnliche Formen gefunden werden, die in den dazwischen liegenden Gegenden nicht vorkommen, so kann man die Ursache hiervon einzig und allein in der ersten Auslese von solchen Gruppen suchen, die überhaupt eine Temperaturerniedrigung ertragen konnten. Aus einem ähnlichen Material konnte durch die Einwirkung ähnlicher Verhältnisse ein verändertes Material gezüchtet werden, das seinen gemeinsamen Ursprung noch erkennen lässt. Die Annahme einer Abnahme der Variationsfähigkeit braucht also nicht gemacht zu werden.

1) Versuch über die erdgeschichtliche Entwickelung der jetzigen Verbreitungsverhältnisse unserer Thierwelt. Hamburg, ISgl 
Allerdings würde es auffallend sein, wenn sich wirklich ein solcher Parallelismus der Formen am Nord- und Südpol fände, da doch jedenfalls, abgesehen von der Temperaturaljnahme, auf cler Nord- und Südseite der Erde vielfach verschiedene Verhältnisse mitgespielt haben. PFEFFER würde zu seinem gezwungenen Erklärungsversuch nicht veranlasst gewesen sein, wenn er das Axiom der "Aehnlichkeit" der arktisch-borealen mit der antarktisch-notialen Fauna nicht aufgestellt hätte. Mir sind kaum Fälle bekannt, wo wirklich ' $z$ wischen nördlichen und südlichen Formen eine derartige Aehnlichkeit auffiele. PFEFFER ', hat die Verbreitung der antarktischen Uferthiere zusammengestellt. Durchmustert man die Liste, so findet man auf den ersten Blick, dass bipolare Gattungen recht wenige vorhanden sind, bipolare Arten zu den Ausnahmen gehören. Abgesehen davon, dass zwischen den bekannten Localitäten, von denen diese Formen nachgewiesen sind, jeden Augenblick vermittehde Fundorte entdeckt werden können, müsste vor allen Dingen untersucht werden, welches der Verwandtschaftsgrad der ,ähnlichen" Formen ist, und darüber ist in den meisten Fällen noch sehr wenig bekannt. Unsere faunistischen und systematischen Kenntnisse sind noch so lückenhaft, dass wir aus derartigen vereinzelten Nachweisen von identischen oder ähnlichen Formen am Nord- und Südpol noch lange nicht von einer "auffallenden Aehnlichkeit" der Fauna beider Gebiete sprechen kïnnen.

Was die Decapoden-Krebse im Speciellen anbelangt, so führt Pfeffer folgende Gattungen an, die nach den Notizen seiner Liste anscheinend eine bipolare Verbreitung haben: Mrunida, Crangon, Hippolyte. Die sonst noch scheinbar hierher gehörigen Gattungen Lithodes und Pandelus sind auszuschliessen, da in der Tiefsee die Verbindung hergestellt ist. MImida ist nicht bipolar, da es auch in den Tropen litorale Arten der Gattung giebt, und sie in der Tiefsee allgemein verbreitet ist. Ebenso ist Hippolyte kosmopolitisch, und gerade die von PFEFFER beschriebene $H$. antarctica ${ }^{2}$ ) schliesst sich durch den II-gliedrigen Carpus der 2. Pereiopoden gerade an die tropischen Arten (gibberosa und marmorata), und nicht an die nordischen $a{ }^{3}$ ). Die von PEEFFer von Süd-Georgien beschriebene Crangon-Art hat allerdings ihre nächsten Verwandten im arktischen Gebiet, die Gattung ist also nach unserer jetzigen Kentniss wirlilich bipolar: ich muss aber darauf hinweisen, dass andere sehr nahe stehende Gattungen der Crangonidac (z. B. Pontophilus) vielfach in der Tiefsee gefunden werden, und es wäre möglich, dass auch für Crangon selbst eint Verbindung in der Tiefsee liegt.

Diesem einzigen Beispiel einer bipolaren Decapoden-Gattung stehen Gruppen gegenüber, die auf das antarktisch-notiale Gebiet beschränlit sind. Ich nenne vor allen die Gattung lasus unter den Palimuridne und fast die ganze Familie der Hymenosomidae. Auch Platyonychus bipustulatus und die Gattung Cyclograpsus sind hierherzurechnen, wenn sie auch Vorstösse nach Norden machen.

Die Unterschiede, die Pfeffer zwischen arktischer und borealer und zwischen antarktischer und notialer Fauna macht, scheinen mir, wenigstens in Bezug auf Decapoden-Krebse, nicht derartige zu sein, dass man diese Gebiete als gleichwerthig z. B. dem Indo-pacifischen coordiniren kann. Ich halte vielmehr das arktische Gebiet nur für einen Theil einer nordisch-circumpolaren Region, welche $i_{n}$ drei Gebiete zerfällt: in das eigentliche arktische, welches eine ziemlich stark ausgesprochene Circumpolarität besitzt, und zwei südliche Ausläufer dieses letzteren, einen im Nord-Atlantic, den anderen im NordPacific. Besonders vom Nord-Atlantic sind gewisse Formen bekannt, die dem eigentlichen arctischen Gebiete fehlen (z. B. Homarus), aber in der Gesammtmasse der Decapoden-Fauna stehen sich Nord-Atlantic und Arctic so nahe, dass man letzteren nur als einen verkümmerten Theil des ersteren auffassen kann. Noch schwieriger ist es, auf der südlichen Halbkugel ein antarktisches und notiales Gebiet zu unterscheiden. An den Südspitzen der Continente und bei den antarktischen Inseln ist thatsächlich eine eigen-

1) Die niedere Thierwelt des antarktischen Ufergebietes, Internat. Polarforsch. Deutsch. Exped., II, I890, p. 520-572.

2) Süd-Georgien, Jahrb. Hamburg. Wiss. Anstalt., IV, ISS7, p. 51, pl. I, fig. 22-27.

3) Ausserdem ist Hippolyte ein Collectivgenus, das offenbar heterogene Elemente enthält. H. gibberosa und marmorata sind als eigene Gattung: Saron von Thallwitz abgetrennt; $H$, antarctica wird jedenfalls auch eine eigene Gattung bilden müssen. 
thümliche Fauna vorhanden, die eine gewisse, aber viel schwächer entwiclielte Circumpolarität zeigt: sonst theilt sich diese Fauna aber in Localfaunen ${ }^{1}$ ).

Wir haben demmach im Litoral der Erde drei Zonen zu unterscheiden, die sich in Folge der kimatischen Differenzirung herausgebildet haben: I) Eine Arktische Region, mit deutlicher Circumpolarität; 2) eine Antarktische Region mit geringerer Circumpolarität und stärkerer Ausprägung von topographischen localen Bezirken, in Folge der dazwischen liegenden grossen Neeresflächen; 3) eine dazwischen liegende dritte Zone, die man als Circumtropische Region bezeichnen könnte, die aber durchaus nicht einheitlich geblieben ist und kaum noch Spuren einer gürtelförmigen Ausbildung erkennen lässt, sondern sich im Gegentheil in besondere Theile getrennt hat, die wir nach der Verschiedenheit ihrer faunistischen Charaktere sehr gut als Regionen hinstellen können, wenn wir uns nur bewusst bleiben, dass diese Regionen einer anderen Ursache ihre Entstehung verdanken, nicht der primären Ursache der klimatischen Absonderung, 'sondern der durch diese erst möglich gewordenen topographischen Trennung.

Die beiden grossen Hauptmassen der Continente, die alte und die neue Welt, reichen nördlich und südlich bis in die Arlitische und Antarlitische Region hinein, andererseits erstrecken sich vom Arctic bis zum Antarctic in meridianer Richtung zwei grosse Wasserflächen, die des Atlantic und des Pacific. Wir haben oben gesehen, dass zunächst Festlandsmassen absolute Schranken für die litoralen Decapoden abgeben, dann aber auch weite Meerestlächen schwer zu überwindende Hindernisse bilden. Wir würden demnach innerhalb des circumtropischen Gürtels vier zusammenhängende Küstenlinien haben, an denen sich getrennt von einander besondere Litoralfaunen entwickeln können: die Ost- und Westseite des (Indo-) Pacific, also einerseits von Süd-Afrika bis Japan, andererseits von Chile bis zur Westküste von NordAmerika, und dann die Ost- und Westseite des Atlantic, einerseits West-Afrika, andererseits Brasilien-WestIndien. Die Erfahrung lehrt aber, dass die Wasserfläche des Atlantic nicht genügt, die beiden letzteren Litoralfaunen zu trennen, dass die litoralen Krebse in ihren pelagischen Larven Verbreitungsmittel besitzen, die ihre Ausbreitung von West-Indien und Brasilien nach West-Afrika oder ungekehrt ermöglichen: WestAfrika besitzt der westindisch-brasilianischen Fauna gegenüber kaum irgendwie bemerkenswerthe eigenthümliche Typen. Im Pacific scheinen jedoch der ungeheuren Breite der Wasserfläche zwischen den pacifischen Inseln und der Westküste Amerikas gegenüber die Verbreitungsmittel der Decapoden im Wesentlichen unwirksam zu sein.

Es bleiben somit nur drei tropische Regionen übrig, die sich den beiden oben genannten als 3., 4. und 5. anreihen: 3) die Atlantische Region, welche die Westküste Afrikas sowie die brasilischen und westindischen Küsten umfasst; 4) die West-amerikanische Region, die Westküste Amerikas von den Vereinigten Staaten bis Chile umfassend; 5) die oben näher begrenzte Indo-pacifische Region ${ }^{2}$ ).

Wir sind aber noch nicht fertig. Es ist kaum anzunehmen, dass diese tropischen Regionen in die nordischen unvermittelt übergehen, sondern wir müssen erwarten, dass dort, wo die Küsten sich in höhere Breiten hinziehen, besondere Verhältnisse eintreten. In Allgemeinen kann man annehmen, dass zwischen tropischen und nordischen Elementen eine allmähliche Vermischung eintritt, zu denen dann weitere Ele-

1) Vgl. Pferfer, Niedere Thierwelt des antarkt. Ufergebietes p. $460 \mathrm{ff}$. Daselbst sind folgende 4 Bezirke genannt: Magellansischer, Süd-Georgischer, Kerguelenischer, Aucklandischer, denen als 5. ein Capischer hinzuzufügen wäre.

2) Diese fünf theoretisch, nach klimatischen und topographischen Grenzen construirten Regionen sind auch durch ihre faunistischen Charaktere, soweit unsere Kenntniss reicht, gut geschieden. Für die drei letzteren ist aber zu bemerken, dass zunächst die west-amerikanische Region noch recht wenig bekannt ist, und ferner, dass alle drei gewisse Beziehungen zu einander zeigen, d. h., dass sie gewisse identische Formen enthalten, die weniger kosmopolitisch, dagegen mehr circumtropisch verbreitet erscheinen. Derartige gemeinsame Züge sind vielleicht aus der geologischen Vorgeschichte dieser Regionen herzuleiten, besonders aus dem Umstande, dass sie in früherer Zeit nicht so vollständig topographisch geschieden waren, wie jetzt. Man kann sich z. B. vorstellen, dass in früherer Zeit noch um die Südspitze Afrikas kerum die Indo-pacifische Fauna mit der atlantischen communiciren konnte, und eine weitere Verbindung dieser beiden Faunen kann vom Rothen zum Mittelmeer existirt haben. (Vgl. KELLER, Die Fauna im Suezkanal etc., I 882, p. 7 ff.) Andererseits ist bekannt, dass zwischen dem atlantischen und west-amerikanischen Litoralgebiet eine topographisclie Verbindung dadurch vorhanden war, dass die Landenge von Panama zur Alt-Tertiärzeit noch nicht existirte: es mögen sich also grewisse gemeinsame Züge genannter Regionen auf diese früheren Zustände zürückführen lassen. Wegen der ausserordentlichen Schwierigkeiten, die derartige Fälle aber darbieten, weil jeder derselben einzeln untersucht werden müsste, und zwar mit genauer Berücksichtigung der systematischen Verwandtschaftsverhältnisse und der paläontologischen Thatsachen, beschränke ich mich hier darauf, nur die Möglichkeit und Wahrscheinlichkeit des Vorhandenseins derartiger Beziehungen anzudeuten. 
mente hinzutreten, die für diese Uebergangsgebiete bezeichnend, als deren Localfauna anzusehen sind. Derartige locale Elemente können sich aber nur da reichhaltig entwickeln, wo die Entwicklung der Kü̈stenlinien, die Flächenausdehnung des Litorals eine besonders ausgedehnte innerhalb dieser intermediären Zonen ist, und nicht da, wo die Küstenlinie einfach und wenig gegliedert ist, und auf eine kurze Strecke hin der Uebergang vom warmen ins kalte Gebiet erfolgt, d. h. wo sich die Küste wesentlich in meridianer. Richtung erstreckt. Und gerade dieses letztere Verhältniss tritt meist in den in Frage kommenden Gebieten ein, nämlich zunächst in der Indo-pacifischen Region dort, wo sich die ost-afrikanische Küste nach Süden ins kalte Gebiet erstreckt, sowie an der West- und Ostküste Australiens, ferner in der Atlantischen Region nach Süden an der Westliüste Afrikas und an der Ostliüste Süd-Amerikas (Brasilien-Argentinien), sowie nach Norden an der Ostküste der Vereinigten Staaten, und schliesslich in den nördlichen und südlichen Grenzgebieten der West-amerilianischen Region. An allen diesen genannten Stellen verlaufen die Küistenlinien im Wesentlichen nord-südlich, sind wenig gegliedert, und es sind somit nicht die Bedingungen gegeben, um reich entwickelte Uebergangs- und Localfaunen sich ausbilden zu lassen, wenn auch solche nicht ganz fehlen mögen. An zwei Stellen der Erde treten aber günstigere Verhältnisse auf: die eine ist der nördliche Grenzbezirk der Atlantischen Region nördlich von der Westlüste Afrikas, wo sich der reich gegliederte Meeresabschnitt des Mittelmeeres befindet, die andere Stelle liegt an der nördlichen Grenze der Indo-pacifischen Region an der Ostlüuste Asiens, wo die japanische Inselwelt ein ungemein reich gegliedertes Litoralgebiet aufweist. Dementsprechend haben sich an beiden Stellen ganz charakteristische Litoralfaunen herausgebildet: die mediterrane und die japanische. Beide sind noch als Theile der sich südlich anschliessenden Atlantischen beziehungsweise Indo-pacifischen Region aufzufassen, nehmen jedoch eine etwas selbständigere Stellung ein, als beliebige andere tropische Gegenden dieser Regionen: als gleichwerthige Regionen lassen sie sich aber nicht abgliedern.

Eine wie oben durchgeführte Begrenzung von marinen litoralen Regionen kann offenbar nicht für alle litoralen Thiergruppen einheitlich durchgeführt werden, und ich muss hier nochmals betonen, dass obige Begrenzung nur für die Decapoden-Krebse gelten soll. Von anderen Thiergruppen werden sich wahrscheinlich einige im Wesentlichen in denselben Rahmen einfügen lassen, es können das aber nur solche sein, die sich den verschiedenen äusseren Lebensbedingungen gegenüber ähnlich verhalten, wie die Decapoden, in ähnlicher Weise sich durch Klima und topographische Verhältnisse in ihrer Ausbreitung behindern lassen und ähnliche Verbreitungsmittel besitzen. Für Thiergruppen, bei denen diese Bedingungen wesentlich anderen Einfluss haben, wird die Verbreitung sich anders gestalten, und es würde eine lohnende Arbeit sein, auch für andere Gruppen derartige Untersuchungen durchzufthren. Auch bei den Decapoden giebt es manche Ausnalmmen: abgesehen von den abyssalen und pelagischen Formen finden sich echte Litoralformen, die sich nicht an die oben angegebenen Grenzen der Regionen halten, und die bestimmte Schranken leichter überwinden können. Derartige Formen finden sich in den verschiedensten Gruppen: es ist aber hervorzuheben, dass sie gegenüber der Mehrzahl der litoralen Decapoden, die sich diesen Grenzen fügen, nur eine verschwindende Anzahl ausmachen.

Zum Schluss muss ich noch ein paar Worte über einige ganz eigenthümliche Gruppen von Decapoden-Krebsen sagen, nämlich über die, welche sich an eine subterrestrische Lebensweise oder an das Leben im Süsswasser gewölnnt haben, da für diese Formen offenbar, gemäss ihrer Abstamnnng von litoralen Formen, ursprünglich die für letztere geltenden Gesetze maassgebend waren, diese aber durch die ver. änderten Lebensbedingungen modificirt werden konnten.

Unter diesen Formen haben wir zwei Hauptgruppen zu unterscheiden: erstens solche, die sich aus Litoralbewohnern direct zu Landbewohnern umwandelten, zweitens solche, die sich ans Leben im Süsswasser gewöhnten, und unter letzteren finden sich wieder gewisse, die terrestrische Gewohnheiten annahmen. 
Einen Uebergang zu den zur ersten Gruppe gehörigen Formen, den Coenobitidae und Gecarcinidae, bilden in ihren Gewohnheiten gewisse Bewohner der Schorre, die sich gerade unter den Verwandten dieser beiden Familien, unter den Paguviden und Grapsiden finden, und die sich in ihrer Verbreitung völlig den litoralen Formen anschliessen. Auch andere Schorrenbewohner, die mit den genannten nicht in näherer Beziehung stehen, haben unabhängig von ihnen sich zu theilweisen Landbewohnern ausgebildet, nämlich die meisten Ocypodiden, besonders die Gattungen Gelusimus und Ocypode. Auch sie schliessen sich in ihrer Verbreitung den echten Litoralformen völlig an. Die Coenobitidae und Gecarcinidae ihrerseits sind als die am allerweitesten ans Landleben angepassten Decapoden anzusehen, und es ist interessant, dass die Coenobitiden sich vollkommen so verhalten, wie litorale Krebse: sie sind circumtropisch und zwar ganz charakteristisch für die Indo-pacifische Region bis auf eine Art, die atlantisch (west-indisch) ist. Bei den Gecarcinidae liegen die Verhältnisse verwickelter: sie lassen zwar den Einfluss der für die Litoralformen geltenden Gesetze noch erkennen, indem bestimmte Arten Indo-pacifisch, andere atlantisch sind, doch überschreiten sie auch, wie es scheint, die Schranke des amerikanischen Continents, was ihnen jedenfalls nur durch ihre Lebensweise ermöglicht wurde.

Andere Gruppen von Decapoden passten sich ans Süssivasser an. Ueber die auffallendste derselben, die Gattung Palaemon, habe ich ${ }^{3}$ ) eine Studie veröffentlicht, in der eine Verbreitung der Gattung nachgewiesen wurde, die im Wesentlichen, allerdings mit einigen interessanten Modificationen, mit der der hitoralen Decapoden sich deckt. Eine andere Süsswassergruppe ist die Familie der Atyidae, die aber besondere Verbreitungsverhältnisse darbietet, welche noch näher zu untersuchen sind. Ganz abweichend verhalten sich aber die Astacidae ${ }^{2}$ ) in ihrer geographischen Vertheilung, und es sind die Ursachen dieser Eigenthümlichkeiten noch nicht mit Sicherheit bekannt. Die auffallendsten Thatsachen sind die, dass die Vertheilung der Familie echt bipolar ist, dass auf jeder Erdhälfte eine eigene Unterfamilie vorkommt, und auch die Gattungen ganz eigenthüimliche Verhältnisse zeigen, besonders auf der nördlichen Halbkugel.

Auf die in anderen Krebsgruppen vereinzelt vorkommenden Süsswasserformen brauche ich hier nicht einzugehen, da sie keine Verbreitungsanomalieen zeigen.

Was schliesslich diejenigen Süsswasserformen anbetrifft, die sich ihrerseits theilsweise ans Landleben gewöhnt haben, die Familie der Thelphusidae und die Unterfamilie der Sesarminae, besonders die Gattung Sesamma, so schliesst sich letztere völlig, soweit mir bekannt, an die litoralen Formen an, während die ersteren Eigenthümlichkeiten zeigen, die einer besonderen Untersuchung bedürfen.

Aus dem allen geht hervor, dass sich bei manchen Land- und Süsswassergruppen ihre Abhängigkeit von den marinen Verbreitungsverhältnissen noch deutlich nachweisen lässt, während bei anderen in Folge der veränderten Lebensweise auch die Gesetze, nach denen sich die Verbreitung gestaltet, einer mehr oder weniger grossen Veränderung unterworfen wurden. Der Schluss dürfte jedenfalls Berechtigung haben, dass diejenigen Süsswasser- und Landformen, die die meisten Eigenthümlichkeiten zeigen und die zu den jetzigen marinen Verbreitungsbezirken keine Beziehungen mehr erkennen lassen, also Formen wie die Atyidae, Astacidae, Thelphusidae, dass diese vor geologischverhältnissmässig langer Zeit, als die jetzigen Zustände des Litorals noch nicht sich ausgebildet hatten, aus dem Litoral auswanderten und sich ans Süsswasserleben anpassten. Diese Familien sind auch als phylogenetisch alte aufzufassen, was für die Astaciden und Atyiden auch morphologisch sich nachweisen lässt, für die Thelphusiden wenigstens beziehungsweise gilt.

I) Zool. Jahrb., V, I891, p. 741-748.

2) Vgl. Huxley, Proceed. Zool. Soc. London, I878, p. 752 ff., und The Crayfisch, 1880. Die speciellen Ansichten HuxLEY'S über die Ursachen dieser eigenthümlichen Verbreitung siehe 1. c. I878, p. 787, und I880, p. 320 ff. und besonders p. 33I ff. Dieselben wurden jedoch beeinflusst durch unzutreffende Vorstellungen über die Classification der Macruren im Allgemeinen, vgl 1. c. 1878, p. $78 \mathrm{I}$ u. 785 . 
Tafel I. 


\section{Tafel I.}

Fig. I. Athanas dimorphus n. spec., of, Seitenansicht, $\frac{6}{1}$.

"Ib. Innere Antenne, 10 .

"Ic. Aeussere Antenne, $\frac{1}{1}^{0}$.

$"$ Id. Mandibel, ca. $\frac{1 "}{1}$.

" I i. Dritter Maxillarfuss, 10 .

" I k. Erster Pereiopode des,$+ \frac{6}{1}$.

"Il. Zweiter Pereiopode, $\frac{10}{1}$.

" I m. Dritter Pereiopode, $\frac{10}{1}$.

"I z. Telson und linker Uropode, ${ }^{10}$.

"2. Caritinn singhalensis nov. spec. Cephalothorax und dessen Gliedmaassen, $\frac{8}{1}$.

"3. Pontonir pimnae nov. spec., $q$, Dorsalansicht, $\frac{a}{1}$.

" 3a. Seitenansicht des Cephalothorax, $\frac{3}{1}$.

" $3 \mathrm{n}$. Endklaue des vierten Pereiopoden, $\frac{10}{1}$.

"4. Mfunida semoni nov. spec., ठ, Dorsalansicht, $\frac{2}{1}$.

"4i. Dritter Maxillarfuss, links, ca. $\frac{4}{1}$.

" 5. Stenorhynchus brevis nov. spec., Dorsalansicht, $\frac{3}{1}$. 


$$
\begin{aligned}
& 5 \\
& y \\
& y
\end{aligned}
$$



Tafel II. 


\section{Tafel II.}

Fig. I. Penaeus monadon FABR.. Thelycum des 2. $\frac{3}{1}$.

. 2. Puer spimiger nor. spec. Sternum, ca. $\frac{5}{1}$.

“ 2i. Dritter Maxillarfuss. ca. $\frac{3}{1}$.

"3. Scyllams elisabethae nor. spec.. Dorsalansicht, $\frac{1}{1}$.

“ 3 a. Seitenansicht. $\frac{1}{1}$.

" +a. Gebia africana nor. spec. Scheere ron innen.

$"$ +b. Scheere ron aussen, $\frac{3}{1}$.

"5. Petrolisthes trivirgatus nov. spec., Dorsalansicht, $\frac{8}{1}$.

"6. Pisisoma gramulatum nor. spec., Dorsalansicht, $\frac{3}{1}$.

"7. Tursia ypsiton nov. spec., Dorsalansicht. $\frac{2}{1}$.

". S. Cryptodromia lamellntr nor. spec., Dorsalansicht, $\frac{2}{1}$. 


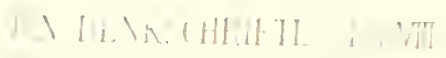
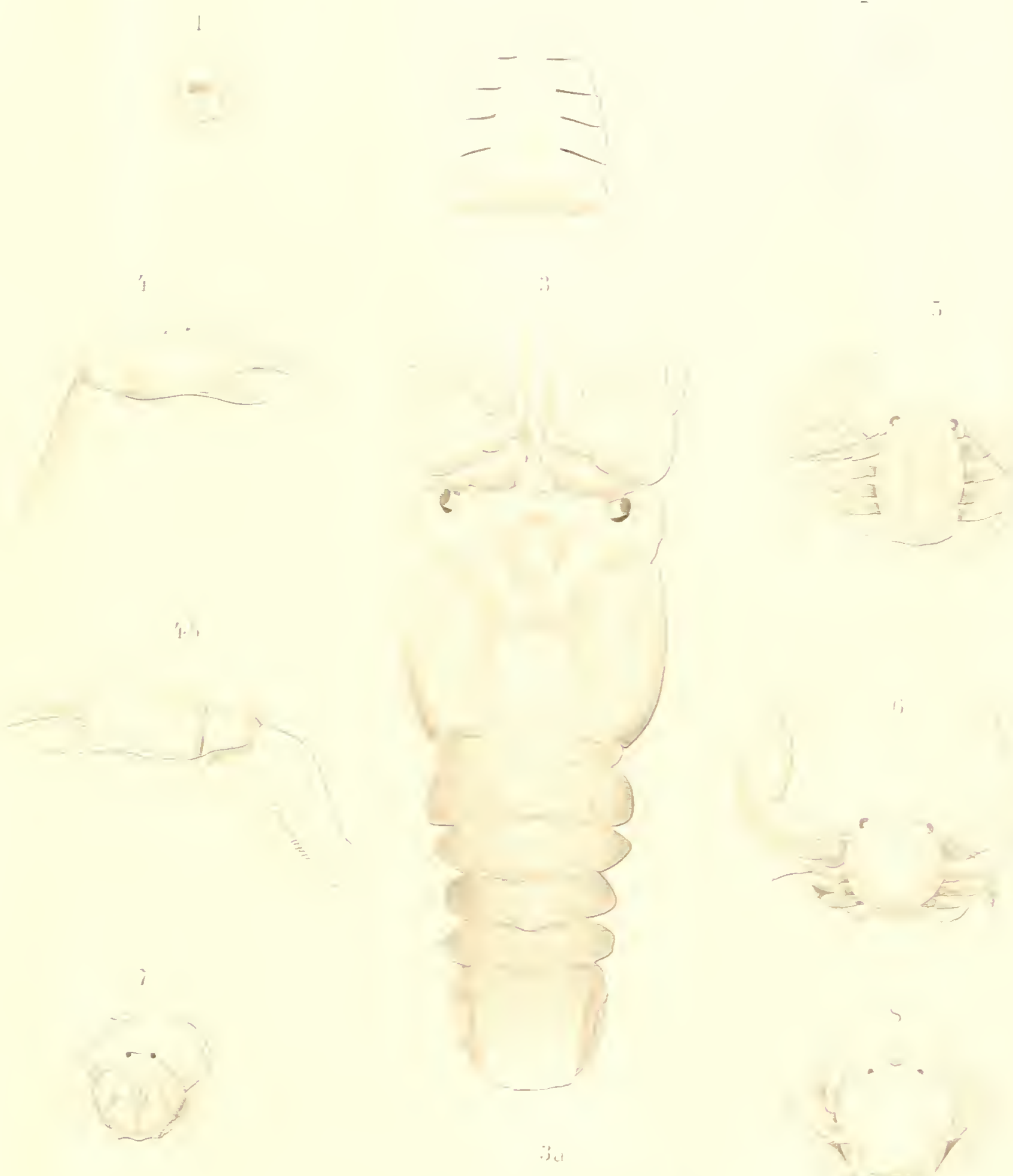
Tafel III. 


\section{Tafel III.}

Fig. I. Gebia (Gebiopsis) hexaceras nov. spec., Stimrand von oben, ca. $\frac{4}{1}$.

"Ik. Rechter Scheerenfuss, von aussen, $\frac{2}{1}$.

" 2. Anacinetops stimpsoni MiERs, Dorsalansicht, ․․ (Behaarung weggelassen.)

" 2 a. Rechte Hälfte des vorderen Theiles des Cephalothorax von unten, ca. 3 .

"3. Hyastemus brevicomis nov. spec., Dorsalansicht, "2. (Behaarung des Cephalothorax und der Beine weggelassen.)

"4. Naxia cerastes nov. spec., Dorsalansicht, $\frac{2}{1}$.

" 4 a. Seitenansicht des Cephalothorax, 2.

" 5. Lambrus (Aulacolambrus) lecanora nov. spec., Dorsalansicht, $\frac{1}{1}$.

"6. Lambrus (Aulacolambms) hoplonotus WHITE, var. dentifrons nov., Stirngegend von oben, $\frac{3}{1 .}$

, 7. Pilummus infraciliaris nov. spec., Dorsalansicht, $\frac{2}{1}$. (Die Behaarung ist auf der einen Hälfte des Cephalothorax weggelassen.)

"7 a. Orbita und angrenzende Theile, von vorn, ca. $\frac{3}{1}$.

"8. Cycloblepas semoni nov. gen. nov. spec., Dorsalansicht, sehr schwach vergr.

"8a. Stirngegend, von vorn gesehen, $\frac{3}{1}$.

" $8 \mathrm{k}$. Rechte Scheere von aussen, ${ }_{1}^{2}$. 
$1.1+11.11$

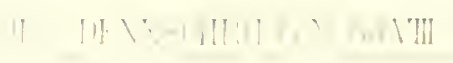
9.11111

$1: 1$

$1 k$

1. al
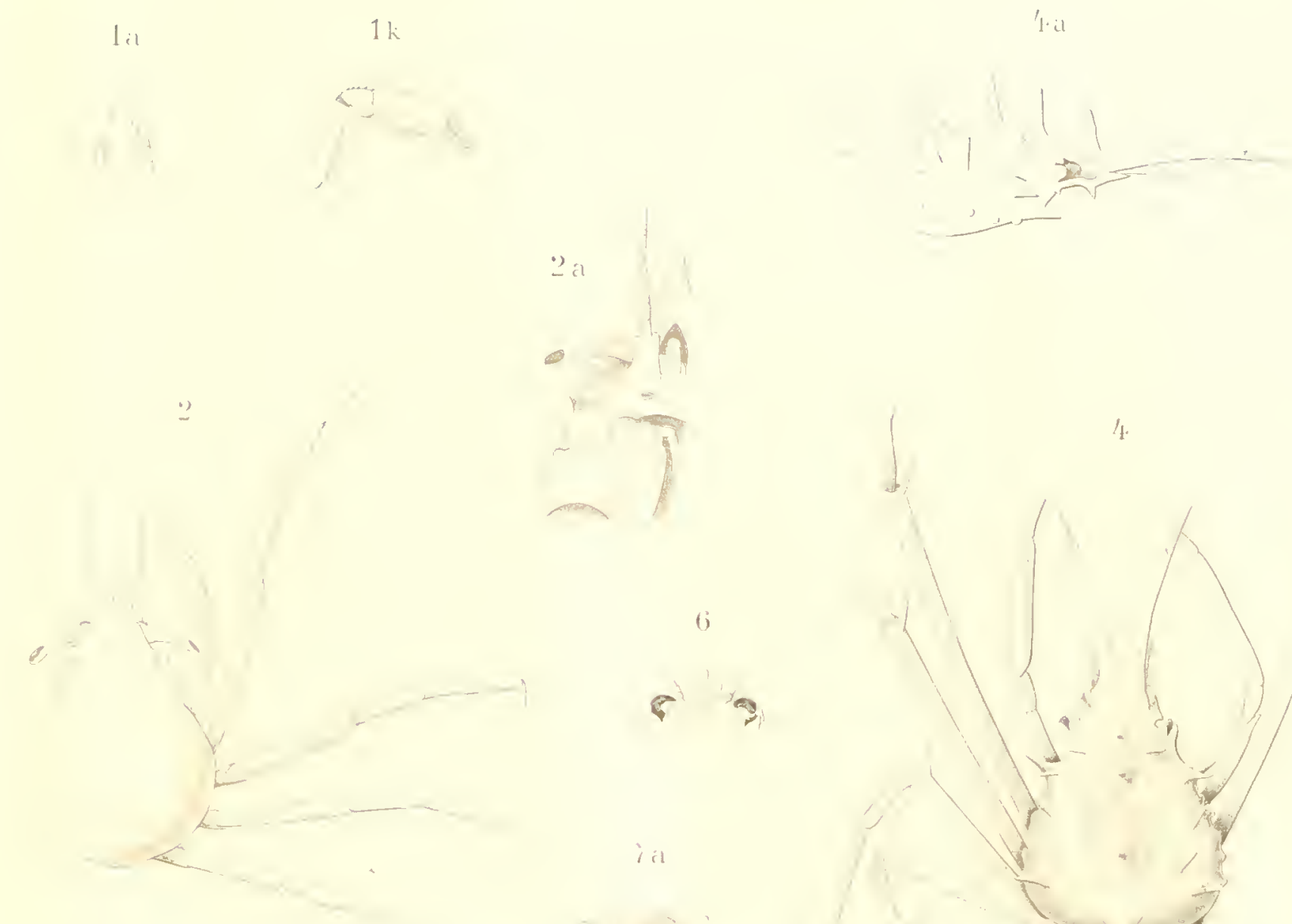

i

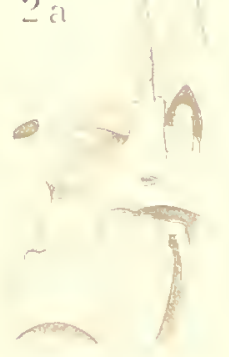

3

;
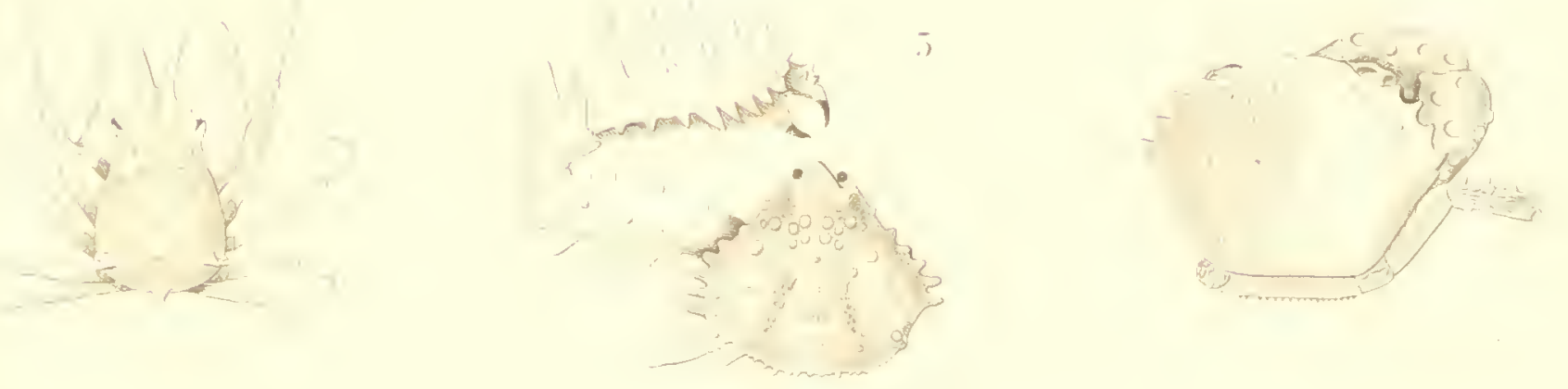

is

Sis

sk

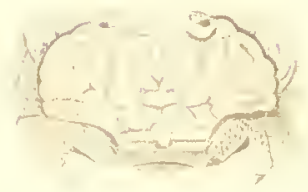

a so 3

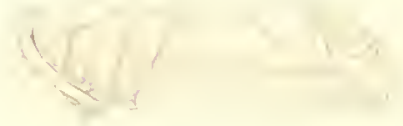






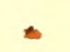



